\title{
On Frobenius and separable algebra extensions in monoidal categories: applications to wreaths
}

\author{
Daniel Bulacu* and Blas Torrecillas** \\ Dedicated to Margaret Beattie on the occasion of her retirement
}

\begin{abstract}
We characterize Frobenius and separable monoidal algebra extensions $i: R \rightarrow S$ in terms given by $R$ and $S$. For instance, under some conditions, we show that the extension is Frobenius, respectively separable, if and only if $S$ is a Frobenius, respectively separable, algebra in the category of bimodules over $R$. In the case when $R$ is separable we show that the extension is separable if and only if $S$ is a separable algebra. Similarly, in the case when $R$ is Frobenius and separable in a sovereign monoidal category we show that the extension is Frobenius if and only if $S$ is a Frobenius algebra and the restriction at $R$ of its Nakayama automorphism is equal to the Nakayama automorphism of $R$. As applications, we obtain several characterizations for an algebra extension associated to a wreath to be Frobenius, respectively separable.
\end{abstract}

Mathematics Subject Classification (2010). 16W30; 16T05, 18D05, 18D10, 16 S34.

Keywords. Monoidal category, 2-category, Frobenius algebra, separable algebra, Nakayama automorphism, wreath product.

\section{Introduction}

Frobenius algebras appeared for the first time in the work of Frobenius on representation theory. These are finite dimensional algebras over a field $k$ having particularly nice duality properties (see for instance the Frobenius equation below). The study of Frobenius algebras was started in the thirties of the last century by Brauer and Nesbitt [3] which named these algebras after Frobenius. Nakayama discovered the duality property of a Frobenius algebra in [35,37], and Dieudonné used this to characterize Frobenius algebras in [15]. Nakayama also studied symmetric algebras in [36] but the automorphism that carries out his name was defined in [37]. Besides representation theory, Frobenius algebras play an important role in number theory,

\footnotetext{
* The first author was supported by the UEFISCDI Grant PN-II-ID-PCE-2011-3-0635, contract no. 253/5.10.2011 of CNCSIS. The first author thanks the Universidad de Almería for its warm hospitality.

${ }^{* *}$ The second author was partially supported by FQM 211 from Junta Andalucía and MTM2011-27090 from MCI. The authors also thank Bodo Pareigis for sharing his "diagrams" program.
} 
algebraic geometry, combinatorics, coding theory, geometry, Hopf algebra and quantum group theory, in finding solutions for the quantum Yang-Baxter equation, the Jones polynomials, etc. More details about the connections between Frobenius algebras and the classical, respectively modern, directions mentioned above can be found in the books of Lam [28] and Kadison [21].

Recently, the interest for Frobenius algebras has been renewed due to connections to monoidal categories and topological quantum field theory (TQFT for short). Roughly speaking, if $\mathbf{n C o b}$ is the category of $n$ cobordisms then a TQFT is a (symmetric) monoidal functor from $\mathbf{n C o b}$ to ${ }_{k} \mathcal{M}$, the category of $k$-vector spaces. For $n=2$ there exists a complete classification of surfaces, and so the cobordism category $\mathbf{2 C o b}$ is described completely. Furthermore, the relations that hold in $\mathbf{2 C o b}$ correspond exactly to the axioms of a commutative Frobenius algebra and this leads to the fact that 2TQFT is equivalent to the category of commutative Frobenius algebras. For more details on this topic we invite the reader to consult [26]. We also note that the Frobenius equation (that follows from the fact that both surfaces are homeomorphic to a sphere with four holes)

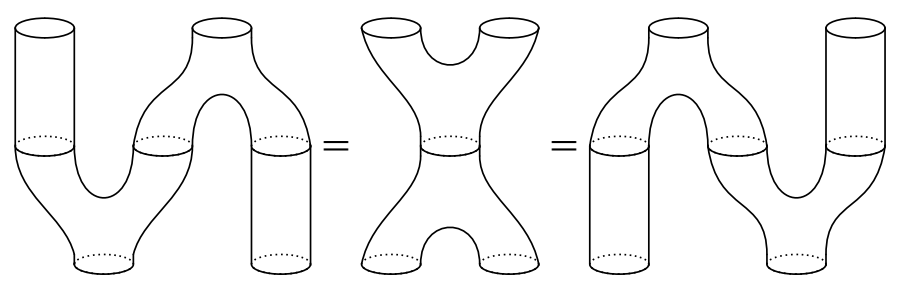

expresses the compatibility between the algebra and coalgebra structure on a Frobenius algebra. It makes sense in any monoidal category, and therefore the notion of Frobenius algebra can be defined in any such category. This has already been done, see for instance [20, 26, 29]. Furthermore, in the monoidal categorical framework Frobenius algebras appear in different contexts. Apart from the TQFT case mentioned above, we have a correspondence between Frobenius algebras in monoidal categories and weak monoidal Morita equivalence of monoidal categories [34], Frobenius functors and Frobenius algebras in the category of endofunctors, and Frobenius monads in 2-categories and Frobenius algebras in some suitable monoidal categories, respectively. Note also that Cayley-Dickson and Clifford algebras are example of Frobenius algebras in certain monoidal categories of graded vector spaces $[4,5]$.

Kasch [23] extended the notion of Frobenius algebra to an arbitrary algebra extension. A $k$-algebra morphism $i: R \rightarrow S$ is called Frobenius if $S$ is finitely generated and projective as right $R$-module and $\operatorname{Hom}_{R}(S, R)$, the set of right $R$-linear maps from $S$ to $R$, is isomorphic to $S$ as $(R, S)$-bimodule. The notion of Frobenius algebra is recovered when $R=k$ and $i$ is the unit map of $S$. We should point out that Frobenius extensions have a well-developed theory of induced representations, investigated in papers by Kasch [22-24], Pareigis [42, 43], 
Nakayama and Tzuzuku [38-40], Morita [32,33], and the list may continue. Frobenius extensions, and so Frobenius algebras as well, can be characterized in terms of Frobenius functors, first introduced by Morita in [32]. Recall that a Frobenius functor is a functor having left and right adjoints which are naturally equivalent, and that the terminology is based on the fact that an algebra extension $i: R \rightarrow S$ is Frobenius if and only if the restriction of scalars functor is Frobenius.

Due to a famous result of Eilenberg and Nakayama [17], particular examples of Frobenius $k$-algebras are given by separable $k$-algebras. Later on, the result was generalized by Endo and Watanabe [18] to the case of algebras over a commutative ring, which are projective as modules. More precisely, they showed that if such an algebra is separable then it is symmetric, and therefore Frobenius. Although the separability notion extends easily to the algebra extension setting, it is still an open question when a separable algebra extension is Frobenius. In this direction the answer is known to be positive in some particular cases, see [50,51]. As far as we are concerned, the separability notion can be restated in terms given by separable functors. These were introduced in [41] and, similar to the Frobenius case, their name is justified by the fact that an algebra extension $i: R \rightarrow S$ is separable if and only if the restriction of scalars functor is separable.

In this paper, which can be seen as a sequel of [8] and a predecessor of [9], we have as final goal the study of the algebra extensions associated to wreaths in monoidal categories from the Frobenius, and respectively separable, point of view. Our motivation is two-fold. On one hand we want to unify most of the Frobenius type theories performed for algebra extensions obtained from different types of entwining structures coming from actions and coactions of Hopf algebras and their generalizations; corroborated with the results and the set of examples presented in [8] this leaded us to the study of wreaths in monoidal categories, and then of algebra extensions produced by them. On the other hand, we wish to give a monoidal categorical interpretation for the conditions under which these algebra extensions are Frobenius, respectively separable, and so to replace a bunch of conditions with one monoidal property. We shall explain this better in what follows by presenting the content of this paper.

Since wreaths in 2-categories are actually monoidal algebras and the monad extensions produced by them are in fact monoidal algebra extensions we first study when a monoidal algebra extension is Frobenius, respectively separable. Having in mind the functorial interpretation that exists in the classical case, we started by investigating when the restriction of scalars functor and the induction functor are Frobenius, respectively separable. To make our theory work, besides the natural conditions that we need to impose (existence of coequalizers, coflatness, robustness), we have to assume that the unit object $\underline{1}$ is a $\otimes$-generator, too. For short, this extra condition is the substitute of the fact that giving an element of a set is equivalent to giving a map from a singleton to the set, or giving a vector is equivalent to giving a linear map from the base field to the vector space where it resides. Otherwise 
stated, we can avoid working with "elements" by considering morphisms in a category having the unit object as source, provided that $\underline{1}$ is a $\otimes$-generator. So under these conditions we give in Theorem 3.5 necessary and sufficient conditions for the restriction of scalars functor to be Frobenius and in Theorem 3.6 the ones for which it is separable, generalizing in this way [10, Theorem 27] to the monoidal categorical setting. As expected, we obtain as a consequence that an algebra $A$ in a monoidal category $\mathcal{C}$ is Frobenius/separable if and only if the forgetful functor $F: \mathcal{C}_{A} \rightarrow \mathcal{C}$ is so, of course, provided that $\underline{1}$ is a $\otimes$-generator in $\mathcal{C}$. Motivated by these results we define in Section 4 the notion of a Frobenius/separable algebra extension in $\mathcal{C}$ in such a way that it becomes equivalent to the fact that the restriction of scalars functor is Frobenius/separable, provided again that $\underline{1}$ is a $\otimes$-generator in $\mathcal{C}$. Our condition on 1 is somehow restrictive and does not apply to every situation, for instance to the category of endofunctors or to the category of (co)representations over a braided bialgebra. Still, there are enough situations when it can be applied or situations when the monoidal category admits another object as $\otimes$-generator, see Examples 3.2. However, we make use of this condition only in the study of when the restriction of scalars functor is Frobenius/separable, the motivation being the one mentioned above. So for the rest of our results this extra assumption on the unit object of the category is not necessary.

If $\underline{1}$ is a projective object in $\mathcal{C}$ then an algebra $A$ in $\mathcal{C}$ is separable if and only if it is projective as an $A$-bimodule in $\mathcal{C}$ (Proposition 4.5). Furthermore, in Proposition 4.8 we show that an algebra extension $i: R \rightarrow S$ is Frobenius/separable if and only if $S$ is a Frobenius/separable algebra in the category of $R$-bimodules in $\mathcal{C},{ }_{R} \mathcal{C}_{R}$, and therefore the study of Frobenius/separable algebra extensions reduce to the study of monoidal Frobenius/separable algebras. Consequently, we get that when $\underline{1}$ is projective or $\otimes$-generator and $R$ is separable the extension $i: R \rightarrow S$ is separable if and only if $S$ is a separable algebra in $\mathcal{C}$ (Corollary 4.9). Note that Proposition 4.8 gives also a new approach for dealing with the problem of when a separable algebra extension is Frobenius. More exactly, if we can answer to question

What are the monoidal categories for which any separable algebra is Frobenius?

then one can uncover the conditions under which a separable algebra extension is Frobenius. However, we can always handle the converse of the above question. More exactly, Proposition 4.10 measures how far is a Frobenius monoidal algebra from being separable, and consequently how far is a Frobenius algebra extension from being separable (Corollary 4.11).

The purpose of Section 5 is to present new characterizations of Frobenius algebra extensions. For an algebra $A$ in a monoidal category, we have several conditions equivalent to $A$ being Frobenius, we collected them in Theorem 5.1. Since an algebra extension $i: R \rightarrow S$ is Frobenius if and only if $S$ is a Frobenius algebra in ${ }_{R} \mathcal{C}_{R}$, we get as an immediate consequence of Theorem 5.1 a list of characterizations for 
$i: R \rightarrow S$ to be Frobenius (Corollary 5.2). The natural problems that show up now are: (1). When is ${ }_{R} \mathcal{C}_{R}$ rigid monoidal?; (2). If ${ }_{R} \mathcal{C}_{R}$ is rigid then is $\mathcal{C}$ so?; and (3). If (2) is true, then how can we relate the dual of an object in ${ }_{R} \mathcal{C}_{R}$ with the dual of the same object regarded now as an object of $\mathcal{C}$ via the forgetful functor? To answer these questions we adapted the techniques used in [55, Section 5], where it is proved that ${ }_{R} \mathcal{C}_{R}$ is rigid in the case when $\mathcal{C}$ is so and $R$ is a special Frobenius algebra (recall that a Frobenius algebra $R$ is called special if $\underline{m}_{R} \underline{\Delta}_{R}=d_{R} \operatorname{Id}_{R}$ and $\underline{\varepsilon}_{R} \underline{\eta}_{R}=d_{R} \operatorname{Id}_{R}$, for some "non-zero scalar" $d_{R}$, where $\left(R, \underline{m}_{R}, \underline{\eta}_{R}\right)$ and $\left(R, \underline{\Delta}_{R}, \underline{\varepsilon}_{R}\right)$ denote the algebra and coalgebra structures of the Frobenius algebra $R$ ). To be more precise, we walked backwards through these questions. Firstly, it is well-known that a strong monoidal functor preserves dual objects, so we might have an answer for (3) in the case when the forgetful functor $\mathfrak{U}:{ }_{R} \mathcal{C}_{R} \rightarrow \mathcal{C}$ is strong monoidal. But this condition on $\mathfrak{U}$ is very restrictive, so we replaced it with the Frobenius monoidal one, a weaker condition under which $\mathfrak{U}$ still preserves dual situations, cf. [13, Theorem 2]. $\mathfrak{U}$ has a trivial monoidal structure and this is part of a Frobenius monoidal structure if and only if $R$ is a Frobenius algebra. Furthermore, if this is the case, then the opmonoidal structure of $\mathfrak{U}$ is completely determined by the Frobenius structure of $R$, see Theorem 6.2. Thus, if $R$ is Frobenius and ${ }_{R} \mathcal{C}_{R}$ is rigid then $\mathcal{C}$ is rigid as well, and this answers partially to (3). Secondly, we prove in Proposition 7.1 that the converse remains true if we assume, in addition, that $R$ is separable (a situation different from the one considered in [55], and more appropriate to the topic of this paper). In particular, if $R$ is a separable Frobenius algebra, we have that ${ }_{R} \mathcal{C}_{R}$ is rigid if and only if $\mathcal{C}$ is so, and if this is the case, the dual objects coincide (only the evaluation and coevaluation morphisms are different). This answers partially the questions (1) and (2). Furthermore, using these results we are able to show that if $R$ is a separable Frobenius algebra, then an algebra extension $i: R \rightarrow S$ is Frobenius if and only if $S$ is a Frobenius algebra and a condition involving the Frobenius structures of $R$ and $S$ holds (Theorem 7.3). When $\mathcal{C}$ is sovereign monoidal, that is, $\mathcal{C}$ is rigid and the left dual functor is equal to the right dual functor, then this condition is equivalent to the the fact that the restriction at $R$ of the Nakayama automorphism of $S$ coincides with the Nakayama automorphism of $R$, see Theorem 7.4.

In Section 8 we give some applications. Particular examples of monoidal algebras are given by monads in an arbitrary category (algebras in a category of endofunctors) and monads in 2-categories. Thus if we specialize our results to these particular situations we get for free necessary and sufficient conditions for which a monad extension or a 2-monad extension is Frobenius, respectively separable. Since the former is a particular case of the latter, we restrict ourselves in working only in the context provided by 2 -categories. If $\mathbb{A}=(A, t, \mu, \eta)$ is a monad in a 2-category $\mathcal{K}$ then $(t, \mu, \eta)$ is an algebra in the monoidal category $\mathcal{K}(A)$ and, moreover, $\mathbb{A}$ is Frobenius/separable in the 2-categorical sense if and only if $(t, \mu, \eta)$ is a monoidal Frobenius/separable algebra in $\mathcal{K}(A)$. With this observation in mind we easily deduce necessary and sufficient conditions for which a wreath, i.e., a 
monad in the 2-category $\operatorname{EM}(\mathcal{K})$ (the Eilenberg-Moore category associated to $\mathcal{K}$ ), is a Frobenius/separable 2-monad. Besides these characterizations, we give also new ones, provided that $\mathcal{K}(A)$ admits coequalizers and any object of it is coflat. More exactly, to any wreath in $\mathcal{K}$ we can associate an algebra extension in $\mathcal{K}(A)$ that we call the canonical monad extension associated to the wreath. Then the wreath is Frobenius/separable if and only if the associated canonical monad extension is so (Theorems 8.6 and 8.7). Finally, all the results obtained throughout the paper can be applied to the (monad) algebra extension associated to a wreath in a monoidal category. We summed up all these in Corollary 8.8 for the Frobenius case, and respectively in Corollary 8.9 for the separable case. In this way we achieved our main goal. Furthermore, we will see in [9] that the Frobenius/separable properties of a monoidal wreath play an important role in establishing Frobenius properties and Maschke type theorems for the generalized entwined module categories that were introduced in [8].

\section{Preliminaries}

2.1. Monoidal categories. Throughout this paper $\mathcal{C}$ is a monoidal category with unit object 1 . Without loss of generality we assume that $\mathcal{C}$ is strict, this means that the associativity and the left and right unit constraints are defined by identity morphisms in $\mathcal{C}$. If $A$ is an algebra in $\mathcal{C}$ we then denote by $\underline{m}_{A}$ and $\underline{\eta}_{A}$ its multiplication and unit morphisms, and by ${ }_{A} \mathcal{C}\left(\mathcal{C}_{A}\right)$ the category of left (right) $A$-modules and left (right) $A$-morphisms in $\mathcal{C}$. For more details about algebras in a monoidal category we invite the reader to consult $[6,25,30,31]$.

Assume now that $\mathcal{C}$ has coequalizers. Take an algebra $A$ in $\mathcal{C}, \mathfrak{M} \in \mathcal{C}_{A}$ and $X \in{ }_{A} \mathcal{C}$, with structure morphisms $\mu_{X}^{A}: A \otimes X \rightarrow X$ and $\nu_{\mathfrak{M}}^{A}: \mathfrak{M} \otimes A \rightarrow \mathfrak{M}$, respectively. We consider the coequalizer $\left(\mathfrak{M} \otimes_{A} X, q_{\mathfrak{M}, X}^{A}\right)$ of the parallel morphisms $\nu_{\mathfrak{M}}^{A} \otimes \operatorname{Id}_{X}$ and $\operatorname{Id}_{\mathfrak{M}} \otimes \mu_{X}^{A}$ in $\mathcal{C}:$

$$
\mathfrak{M} \otimes A \otimes X \underset{\mathrm{Id}_{\mathfrak{M}} \otimes \mu_{X}^{A}}{\stackrel{v_{\mathfrak{M}}^{A} \otimes \mathrm{Id}_{X}}{\longrightarrow}} \mathfrak{M} \otimes X \stackrel{q_{\mathfrak{M}, X}^{A}}{\longrightarrow} \mathfrak{M} \otimes_{A} X .
$$

For a left $A$-linear morphism $f: X \rightarrow Y$ in $\mathcal{C}$, let $\tilde{f}: \mathfrak{M} \otimes_{A} X \rightarrow \mathfrak{M} \otimes_{A} Y$ be the unique morphism in $\mathcal{C}$ satisfying the equation

$$
\tilde{f} q_{\mathfrak{M}, X}^{A}=q_{\mathfrak{M}, Y}^{A}\left(\operatorname{Id}_{\mathfrak{M}} \otimes f\right) .
$$

Take $X \stackrel{g}{\longrightarrow} Y \stackrel{f}{\longrightarrow} Z$ in ${ }_{A} \mathcal{C}$. It is easily verified that $\widetilde{g f}=\tilde{g} \tilde{f}$.

Now let $g: \mathfrak{M} \rightarrow \mathfrak{N}$ in $\mathcal{C}_{A}$ and $Y \in{ }_{A} \mathcal{C}$. Then $\hat{g}: \mathfrak{M} \otimes_{A} Y \rightarrow \mathfrak{N} \otimes_{A} Y$ denotes the unique morphism in $\mathcal{C}$ obeying

$$
\hat{g} q_{\mathfrak{M}, Y}^{A}=q_{\mathfrak{N}, Y}^{A}\left(g \otimes \operatorname{Id}_{Y}\right) .
$$

For $\mathfrak{M} \stackrel{f}{\longrightarrow} \mathfrak{N} \stackrel{g}{\longrightarrow} \mathfrak{P}$ in $\mathcal{C}_{A}$, we have that $\widehat{g f}=\hat{g} \hat{f}$. 
For $\mathfrak{M} \in \mathcal{C}_{A}, X \in \mathcal{C}$ and $Y \in{ }_{A} \mathcal{C}$, we have canonical isomorphisms $\Upsilon_{\mathfrak{M}}, \Upsilon_{\mathfrak{M}, X}$ and $\Upsilon_{Y}^{\prime}$ :

- $\Upsilon_{\mathfrak{M}}: \mathfrak{M} \otimes_{A} A \stackrel{\cong}{\longrightarrow} \mathfrak{M}$, uniquely determined by the property $\Upsilon_{\mathfrak{M}} q_{\mathfrak{M}, A}^{A}=v_{\mathfrak{M}}^{A}$;

- $\Upsilon_{\mathfrak{M}, X}: \mathfrak{M} \otimes_{A}(A \otimes X) \stackrel{\cong}{\longrightarrow} \mathfrak{M} \otimes X$, uniquely determined by the property $\Upsilon_{\mathfrak{M}, X} q_{\mathfrak{M}, A \otimes X}^{A}=v_{\mathfrak{M}}^{A} \otimes \operatorname{Id}_{X}$

- $\Upsilon_{Y}^{\prime}: A \otimes_{A} Y \stackrel{\cong}{\longrightarrow} Y$, uniquely determined by the property $\Upsilon_{Y}^{\prime} q_{A, Y}^{A}=\mu_{Y}^{A}$.

The following properties are now easily verified:

$$
\begin{gathered}
\Upsilon_{\mathfrak{M}}^{-1}=q_{\mathfrak{M}, A}^{A}\left(\operatorname{Id}_{\mathfrak{M}} \otimes \underline{\eta}_{A}\right) ; \quad \Upsilon_{Y}^{\prime-1}=q_{A, Y}^{A}\left(\underline{\eta}_{A} \otimes \operatorname{Id}_{Y}\right) ; \\
\Upsilon_{\mathfrak{M}, X}^{-1}=q_{\mathfrak{M}, A \otimes X}^{A}\left(\operatorname{Id}_{\mathfrak{M}} \otimes \underline{\eta}_{A} \otimes \operatorname{Id}_{X}\right) .
\end{gathered}
$$

Next, recall that an object $X$ of $\mathcal{C}$ is called right (left) coflat if the functor $X \otimes-$ (respectively $-\otimes X$ ) preserves coequalizers. An object of $\mathcal{C}$ is called coflat if it is both left and right coflat.

Let now $\mathcal{C}$ be a monoidal category with coequalizers and $A, R$ algebras in $\mathcal{C}$. By [7, Lemma 2.4] we have the following.

(a) If $A$ is right coflat then for any $X \in{ }_{A} \mathcal{C}_{R}$ and $Y \in{ }_{R} \mathcal{C}$ the morphism $\mu_{X \otimes_{R} Y}^{A}: A \otimes X \otimes_{R} Y \rightarrow X \otimes_{R} Y$ uniquely determined by $\mu_{X \otimes_{R} Y}^{A}\left(\operatorname{Id}_{A} \otimes q_{X, Y}^{R}\right)=$ $q_{X, Y}^{R}\left(\mu_{X}^{A} \otimes \operatorname{Id}_{Y}\right)$ defines on $X \otimes_{R} Y$ a left $A$-module structure in $\mathcal{C}$, where, in general, by $\mu_{X}^{A}: A \otimes X \rightarrow X$ we denote a left $A$-module structure on an object $X$ of $\mathcal{C}$;

(b) Likewise, if $A$ is left coflat then for any $X \in \mathcal{C}_{R}$ and $Y \in{ }_{R} \mathcal{C}_{A}$ the morphism $v_{X \otimes_{R} Y}^{A}: X \otimes_{R} Y \otimes A \rightarrow X \otimes_{R} Y$ uniquely determined by $v_{X \otimes_{R} Y}^{A}\left(q_{X, Y}^{R} \otimes \operatorname{Id}_{A}\right)=$ $q_{X, Y}^{R}\left(\operatorname{Id}_{X} \otimes v_{Y}^{A}\right)$ defines on $X \otimes_{R} Y$ a right $A$-module structure in $\mathcal{C}$, where, in general, by $v_{Y}^{A}: Y \otimes A \rightarrow Y$ we denote the structure morphism of a right $A$ module $Y$ in $\mathcal{C}$.

If $R$ is an algebra in $\mathcal{C}$ we then say that an object $Y \in{ }_{R} \mathcal{C}$ is left robust if for any $\mathfrak{M} \in \mathcal{C}, X \in \mathcal{C}_{R}$ the morphism $\theta_{\mathfrak{M}, X, Y}^{\prime}:(\mathfrak{M} \otimes X) \otimes_{R} Y \rightarrow \mathfrak{M} \otimes\left(X \otimes_{R} Y\right)$ defined by the commutativity of the diagram

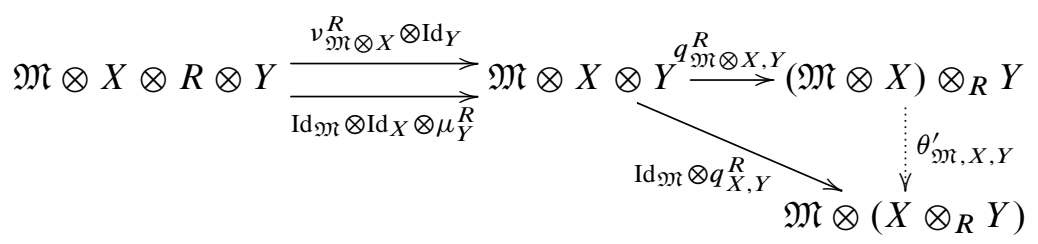

is an isomorphism. If $R$ is left coflat then it is well known that the category of $R$ bimodules in $\mathcal{C}$ that are left coflat and left robust, denoted by ${ }_{R} \mathcal{C}_{R}$, is a monoidal 
category (see for instance $[7,44,46]$ ). Notice that the left robustness of an object $Y \in{ }_{R} \mathcal{C}$ is needed in order to define

(1) a left $R$-module structure on $X \otimes_{R} Y$ in $\mathcal{C}$, for any $X \in{ }_{R} \mathcal{C}_{R}$. Namely, if we define $\bar{\mu}_{X \otimes_{R} Y}:(R \otimes X) \otimes_{R} Y \rightarrow X \otimes_{R} Y$ as being the unique morphism in $\mathcal{C}$ making the diagram

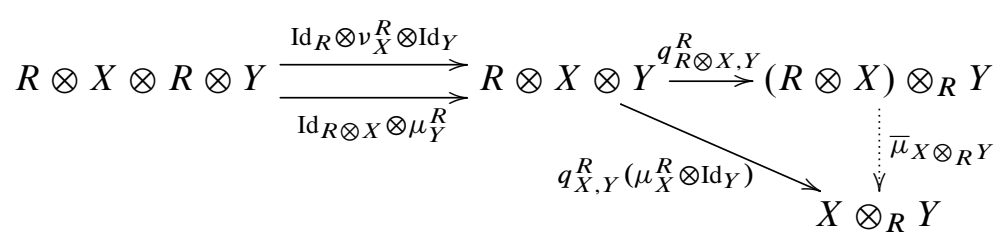

commutative then the morphism $\mu_{X \otimes_{R} Y}^{R}:=\bar{\mu}_{X \otimes_{R} Y} \theta_{\mathfrak{M}, X, Y}^{\prime-1}$ defines on $X \otimes_{R} Y$ a left $R$-module structure in $\mathcal{C}$. Furthermore, $X \otimes_{R} Y$ becomes in this way an $R$ bimodule, provided that $Y \in{ }_{R} \mathcal{C}_{R}$ and $R$ is left coflat. We should also remark that this left $R$-module structure coincide with that when $R$ is right coflat, see $(a)$ above;

(2) a morphism $\Gamma_{\mathfrak{M}, X, Y}^{\prime}: \mathfrak{M} \otimes_{R}\left(X \otimes_{R} Y\right) \rightarrow\left(\mathfrak{M} \otimes_{R} X\right) \otimes_{R} Y$, for all $\mathfrak{M}, X, Y \in{ }_{R} \mathcal{C}_{R}$, uniquely determined by $\Gamma_{\mathfrak{M}, X, Y}^{\prime} q_{\mathfrak{M}, X \otimes_{R} Y}^{R}=\widehat{q_{\mathfrak{M}, X}^{R}} \theta_{\mathfrak{M}, X, Y}^{\prime-1}$. It is actually an isomorphism with inverse $\Sigma_{\mathfrak{M}, X, Y}^{\prime}$ uniquely determined by the property that

$$
\Sigma_{\mathfrak{M}, X, Y}^{\prime} q_{\mathfrak{M} \otimes_{R} X, Y}^{R}\left(q_{\mathfrak{M}, X}^{R} \otimes \operatorname{Id}_{Y}\right)=q_{\mathfrak{M}, X \otimes_{R} Y}^{R}\left(\operatorname{Id}_{\mathfrak{M}} \otimes q_{X, Y}^{R}\right) .
$$

So when $R$ is left coflat the category $!_{R} \mathcal{C}_{R}$ is monoidal with tensor product $\otimes_{R}$, associativity constraint $\Sigma_{-,-,-}^{\prime}$, unit object $R$, and left and right unit constraints $\Upsilon_{-}^{\prime}$ and $\Upsilon_{-}$. Once more, the full details can be found in $[7,44,46]$.

Throughout this paper when we make use of the monoidal structure of ${ }_{R} \mathcal{C}_{R}$ it is implicitly understood that $\mathcal{C}$ has coequalizers, and that any object of it is coflat and left robust. In other words we identify the categories ${ }_{R} \mathcal{C}_{R}$ and ${ }_{R} \mathcal{C}_{R}$. The aim is to make our results more readable and less technical.

2.2. Monads in 2-categories. Let $\mathcal{K}$ be a 2-category; its objects (or 0-cells) will be denoted by capital letters. 1-cell between two 0-cells $U$ and $V$ will be denoted as $U \stackrel{f}{\longrightarrow} V$, the identity morphism of a 1-cell $f$ by $1_{f}$ and, more generally, a 2-cell by $f \stackrel{\rho}{\Longrightarrow} f^{\prime}$. We also denote by $\circ$ the vertical composition of 2-cells $f \stackrel{\rho}{\Longrightarrow} f^{\prime} \stackrel{\tau}{\Longrightarrow} f^{\prime \prime}$ in $\mathcal{K}(U, V)$, by $\odot$ the horizontal composition of 2-cells

$$
U \underset{\frac{f^{\prime}}{\underset{\Downarrow \rho \rho}{\longrightarrow}}}{\stackrel{f}{\longrightarrow}} V \frac{g}{\frac{\Downarrow \rho^{\prime}}{g^{\prime}}} W, \quad g \circ f \stackrel{\rho^{\prime} \odot \rho}{\Longrightarrow} g^{\prime} \circ f^{\prime},
$$

and by $\left(U \stackrel{1_{U}}{\longrightarrow} U, 1_{U} \stackrel{i_{U}}{\Longrightarrow} 1_{U}\right)$ the pair defined by the image of the unit functor from $\mathbf{1}$ to $\mathcal{K}(U, U)$, where $\mathbf{1}$ is the terminal object of the category of small 
categories. For more details on 2-categories, we refer the reader to [1, Ch. 7] or [31, Ch. XII].

A monad in $\mathcal{K}$ is a quadruple $(A, t, \mu, \eta)$ consisting of an object $A$ of $\mathcal{K}$, a 1-cell $A \stackrel{t}{\longrightarrow} A$ in $\mathcal{K}$ and 2-cells $t \circ t \stackrel{\mu}{\Longrightarrow} t$ and $1_{A} \stackrel{\eta}{\Longrightarrow} t$ in $\mathcal{K}$ such that

$$
\mu \circ\left(\mu \odot 1_{t}\right)=\mu \circ\left(1_{t} \odot \mu\right), \mu \circ\left(1_{t} \odot \eta\right)=1_{t}=\mu \circ\left(\eta \odot 1_{t}\right) .
$$

If $\mathbb{A}=\left(A, t, \mu_{t}, \eta_{t}\right)$ and $\mathbb{B}=\left(B, s, \mu_{s}, \eta_{s}\right)$ are monads in $\mathcal{K}$ then a monad morphism between $\mathbb{A}$ and $\mathbb{B}$ is a pair $(f, \psi)$ with $A \stackrel{f}{\longrightarrow} B$ a 1-cell in $\mathbb{K}$ and $s \circ f \stackrel{\psi}{\Longrightarrow} f \circ t$ a 2-cell in $\mathcal{K}$ such that the following equalities hold:

$$
\left(1_{f} \odot \mu_{t}\right) \circ\left(\psi \odot 1_{t}\right) \circ\left(1_{s} \odot \psi\right)=\psi \circ\left(\mu_{s} \odot 1_{f}\right), \psi \circ\left(\eta_{s} \odot 1_{f}\right)=1_{f} \odot \eta_{t} .
$$

2.3. Frobenius and separable functors. Let $\mathcal{F}$ be a functor between two arbitrary categories $\mathcal{D}$ and $\mathcal{E}$. Recall that $\mathcal{F}$ is called Frobenius if it has a right adjoint functor which is also left adjoint, and that $\mathcal{F}$ is called separable if for any two objects $X, Y$ of $\mathcal{D}$ there exists a map $\mathcal{P}_{X, Y}: \operatorname{Hom}_{\mathcal{E}}(\mathcal{F}(X), \mathcal{F}(Y)) \rightarrow \operatorname{Hom}_{\mathcal{D}}(X, Y)$ such that $\mathcal{P}_{X, Y}(\mathcal{F}(f))=f$, for all $f: X \rightarrow Y$ in $\mathcal{D}$, and $\mathcal{P}_{Y_{1}, Y_{2}}\left(g_{2}\right) \circ f_{1}=f_{2} \circ \mathcal{P}_{X_{1}, X_{2}}\left(g_{1}\right)$ for every commutative diagram in $\mathcal{E}$ of type

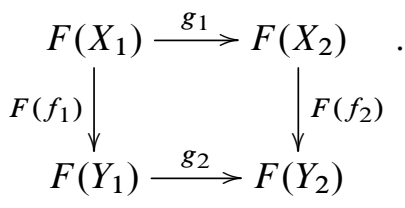

When $\mathcal{F}$ has a right adjoint $\mathcal{G}: \mathcal{E} \rightarrow \mathcal{D}$ the Rafael's theorem [45] gives necessary and sufficient conditions for $\mathcal{F}$ or $\mathcal{G}$ to be separable. More exactly, if $1_{\mathcal{D}}$ and $1_{\mathcal{E}}$ are the identity functors on $\mathcal{D}$ and $\mathcal{E}$, and $\eta: 1_{\mathcal{D}} \rightarrow \mathcal{G F}$ and $\varepsilon: \mathcal{F} \mathcal{G} \rightarrow 1_{\mathcal{E}}$ are the unit and the counit of the adjunction, respectively, then

- $\mathcal{F}$ is separable if and only if the unit $\eta$ splits, that is there exists a natural transformation $v: \mathcal{G F} \rightarrow 1_{\mathcal{D}}$ such that $v \circ \eta$ is the identity natural transformation of $1_{\mathcal{D}}$

- $\mathcal{G}$ is separable if and only if the counit $\varepsilon$ cosplits, that is there exists a natural transformation $\mu: 1_{\mathcal{E}} \rightarrow \mathcal{F} \mathcal{G}$ such that $\varepsilon \circ \mu$ is the identity natural transformation of $1_{\mathcal{E}}$.

\section{Frobenius and separable type properties for the restriction of scalars functor and the induction functor}

In the literature there are several Frobenius or separable theories developed for different kinds of algebras. All these are based on the fact that a certain canonical 
functor is Frobenius, respectively separable. Usually, this canonical functor is a forgetful functor or, more generally, a functor restriction of scalars.

As far as we are concerned, we are interested to study when the extension defined by a wreath in a monoidal category $\mathcal{C}$ is Frobenius, respectively separable. This can be done in two ways, depending on the point of view: monoidal or 2-categorical. As we will see, both cases require actually the study of Frobenius (respectively separable) algebra extensions in a monoidal category, and this is why we shall study this problem first. More precisely, for an algebra extension $S / R$ in $\mathcal{C}$, that is for an algebra morphism $i: R \rightarrow S$ in a monoidal category $\mathcal{C}$, we will give necessary and sufficient conditions for which the functor restriction of scalars $F: \mathcal{C}_{S} \rightarrow \mathcal{C}_{R}$ is Frobenius, and respectively separable.

The functor restriction of scalars $F$ has always a left adjoint functor $G$. Namely, $G$ is the induction functor $-\otimes_{R} S: \mathcal{C}_{R} \rightarrow \mathcal{C}_{S}$ which is defined as follows. If $X \in \mathcal{C}_{R}$ then $\left(-\otimes_{R} S\right)(X)=X \otimes_{R} S$ endowed with the right $S$-module structure induced by the multiplication of $S$, and if $f: X \rightarrow Y$ is a morphism in $\mathcal{C}_{R}$ then $\left(-\otimes_{R} S\right)(f)=\hat{f}$. The unit and the counit of the adjunction are described as follows, for all $X \in \mathcal{C}_{R}$ and $\mathfrak{M} \in \mathcal{C}_{S}$,

$$
\eta_{X}:=q_{X, S}^{R}\left(\operatorname{Id}_{X} \otimes \underline{\eta}_{S}\right): X \rightarrow X \otimes_{R} S, \quad \varepsilon_{\mathfrak{M}}:=\overline{v_{\mathfrak{M}}^{S}}: \mathfrak{M} \otimes_{R} S \rightarrow \mathfrak{M},
$$

the latter being determined uniquely by the property that $\overline{v_{\mathfrak{M}}^{S}} q_{\mathfrak{M}, S}^{R}=v_{\mathfrak{M}}^{S}$.

So $F$ is a Frobenius functor if and only if $G=-\otimes_{R} S$ is a right adjoint functor of $F$, and $F$ is separable if and only if the counit of the adjunction defined above splits. To see when these conditions hold we first describe the sets $\operatorname{Nat}\left(F \circ\left(-\otimes_{R} S\right),-\right)$ and $\operatorname{Nat}\left(-,\left(-\otimes_{R} S\right) \circ F\right)$, where, in general, if $\mathcal{F}, \mathcal{G}: \mathcal{D} \rightarrow \mathcal{E}$ are two functors we then denote by $\operatorname{Nat}(\mathcal{F}, \mathcal{G})$ the set of natural transformations from $\mathcal{F}$ to $\mathcal{G}$. To this end we use the techniques performed in [10]. To make them work in the setting of a monoidal category $\mathcal{C}$ we have to assume that $\mathcal{C}$ is left $\otimes$-generated by its unit object. This means the following.

Definition 3.1. Let $\mathcal{C}$ be a monoidal category. We say that an object $P$ of $\mathcal{C}$ is a left $\otimes$-generator of $\mathcal{C}$ if wherever we consider two morphisms $Y \otimes Z \stackrel{f}{\underset{g}{\longrightarrow}} W$ in $\mathcal{C}$ such that $f\left(\epsilon \otimes \operatorname{Id}_{Z}\right)=g\left(\epsilon \otimes \operatorname{Id}_{Z}\right)$, for all $\epsilon: P \rightarrow Y$ in $\mathcal{C}$, we then have $f=g$.

Observe that, by taking $Z=\underline{1}$ in the above definition we get that a left $\otimes$ generator of a monoidal category $\mathcal{C}$ is necessarily a generator for $\mathcal{C}$, and this justifies our terminology.

Examples 3.2. 1) The category Sets of sets and functions is monoidal with the tensor product the Cartesian product and the unit object a one element set, say $\{*\}$. It can be easily seen that $\{*\}$ is a left $\otimes$-generator.

This example can be widely generalized to the category Top of topological spaces and continuous mappings. For this, observe that the continuous mappings from 
a singleton to a space $Y$ correspond precisely with the points of $Y$, and that two continuous mappings having the same source and target spaces are equal precisely when they coincide on each point of their commune source space.

2) Let $k$ be a commutative ring and denote by ${ }_{k} \mathcal{M}$ the category of left $k$-modules and left $k$-linear maps. It is well-known that ${ }_{k} \mathcal{M}$ is monoidal with tensor product $\otimes=\otimes_{k}$, unit $\underline{1}=k$ and the obvious morphisms $a, l, r$. Since a morphism $\epsilon: k \rightarrow Y$ in ${ }_{k} \mathcal{M}$ is uniquely determined by an element of $Y$ it follows that $\underline{1}$ is a left $\otimes$-generator.

In particular, if $\mathbf{A b}$ denotes the category of abelian groups and group morphisms then $\left(\mathbf{A b}, \otimes_{\mathbb{Z}}, \mathbb{Z}\right)$ is monoidal and its unit object $\mathbb{Z}$ is a left $\otimes$-generator. Here $\mathbb{Z}$ is the ring of integers.

3) It is a generalization of the example 2) to the noncommutative case. Let $k$ be a commutative ring and $R$ a $k$-algebra, and denote by ${ }_{R} \mathcal{M}_{R}$ the category of $R$-bimodules and $R$-bilinear morphisms. We know that ${ }_{R} \mathcal{M}_{R}$ is monoidal with $\otimes=\otimes_{R}, \underline{1}=R$ and the natural morphisms $a, l, r$. We claim that $R$ is a left $\otimes$-generator if and only if $R$ is a generator in ${ }_{R} \mathcal{M}_{R}$, and that this situation occurs when $R$ is a separable $k$-algebra, that is, a separable algebra in ${ }_{k} \mathcal{M}$ (see Definition 4.1 below).

Indeed, we have seen that, in general, a $\otimes$-generator is a generator for the underlying category. For the converse, assume that $R$ is a generator in ${ }_{R} \mathcal{M}_{R}$ and identify ${ }_{R} \mathcal{M}_{R}$ with the category $R^{e} \mathcal{M}$ of left $R^{e}:=R \otimes R^{\text {op }}$-modules in the canonical way (unadorned $\otimes$ means $\otimes_{k}$ ). Then by [28, (18.8) Theorem] the fact that $R$ is a generator in $R^{e} \mathcal{M}$ is equivalent to the existence of some $R$-bilinear morphisms $f_{1}, \cdots, f_{n}: R \rightarrow R^{e}$ and of some elements $x_{1}, \cdots, x_{n} \in R$ such that $\sum_{i=1}^{n} f_{i}\left(x_{i}\right)=1 \otimes 1$. Since any morphism $f_{i}$ is completely determined by an element $e_{i}=e_{i}^{1} \otimes e_{i}^{2} \in R \otimes R$ (summation implicitly understood) obeying $a e_{i}^{1} \otimes e_{i}^{2}=e_{i}^{1} \otimes e_{i}^{2} a$, for all $a \in R$, it follows that $R$ is a generator in ${ }_{R} \mathcal{M}_{R}$ if and only if there exist $e_{i}^{1} \otimes e_{i}^{2} \in R \otimes R$ and $x_{i} \in R, 1 \leq i \leq n$, such that, for all $a \in R$,

$$
a e_{i}^{1} \otimes e_{i}^{2}=e_{i}^{1} \otimes e_{i}^{2} a, \quad \forall 1 \leq i \leq n, \text { and } \sum_{i=1}^{n} x_{i} e_{i}^{1} \otimes e_{i}^{2}=1 \otimes 1 .
$$

Let now $Y \otimes_{R} Z \underset{g}{\stackrel{f}{\longrightarrow}} W$ be morphisms in ${ }_{R} \mathcal{M}_{R}$ such that $f\left(\epsilon \otimes_{R} \operatorname{Id}_{Z}\right)=$ $g\left(\epsilon \otimes_{R} \operatorname{Id}_{Z}\right)$, for all $\epsilon: R \rightarrow Y$ in ${ }_{R} \mathcal{M}_{R}$. Fix an element $y \in Y$ and for any $1 \leq i \leq n$ take $\epsilon_{i}: R \rightarrow Y$ given by $\epsilon_{i}(r)=r e_{i}^{1} \cdot y \cdot e_{i}^{2}$, for all $r \in R$. Since $\epsilon_{i}$ is a morphism in ${ }_{R} \mathcal{M}_{R}$ we get that, for all $i$ 's, $r \in R, y \in Y$ and $z \in Z$,

$$
f\left(r e_{i}^{1} \cdot y \cdot e_{i}^{2} \otimes_{R} z\right)=g\left(r e_{i}^{1} \cdot y \cdot e_{i}^{2} \otimes_{R} z\right) \Leftrightarrow f\left(r e_{i}^{1} \cdot y \otimes_{R} e_{i}^{2} \cdot z\right)=g\left(r e_{i}^{1} \cdot y \otimes_{R} e_{i}^{2} \cdot z\right) .
$$


Therefore, taking $r=x_{i}$ and summing up we obtain that

$$
f\left(\sum_{i=1}^{n} x_{i} e_{i}^{1} \cdot y \otimes_{R} e_{i}^{2} \cdot z\right)=g\left(\sum_{i=1}^{n} x_{i} e_{i}^{1} \cdot y \otimes_{R} e_{i}^{2} \cdot z\right) .
$$

By (3.2) we deduce that $f\left(y \otimes_{R} z\right)=g\left(y \otimes_{R} z\right)$, for all $y \in Y$ and $z \in Z$, as needed.

Finally, it is well-known that $R$ is a generator in ${ }_{R} \mathcal{M}_{R}$ if and only if $R$ is an Azumaya algebra, i.e., a separable algebra over its center $Z(R)$ (for instance, see [56, 28.1\& 28.7]). Now, if $R$ is a separable $k$-algebra then $R$ is a separable $Z(R)$-algebra as well, since the natural mapping $R \otimes R \rightarrow R \otimes_{Z(R)} R$ pulls back the splitting map for the multiplication of $R$. Hence $R$ is both a generator and a left $\otimes_{R}$-generator for ${ }_{R} \mathcal{M}_{R}$, as claimed.

4) Denote by FdHilb the category which has complex finite dimensional Hilbert spaces as objects and continuous linear maps as morphisms. Note that, since the objects of FdHilb are finite dimensional vector spaces it follows that the morphisms in FdHilb are actually the $\mathbb{C}$-linear maps, where $\mathbb{C}$ is the field of complex numbers. Also, the completeness condition with respect to the norm defined by the inner product is insignificant for finite dimensional Hilbert spaces.

FdHilb is monoidal with the monoidal structure inherited from the monoidal structure of $\mathbb{C} \mathcal{M}$ : it is a strict monoidal category with $\otimes=\otimes_{\mathbb{C}}$ and $\underline{1}=\mathbb{C}$. Since we can regard vectors in a finite dimensional Hilbert space $H$ as morphisms from

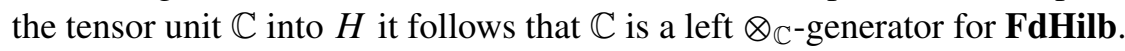

5) Let $k$ be a field and $\mathcal{Z}_{k}$ the category of Zunino over $k$, [11]. Objects of $\mathcal{Z}_{k}$ are pairs $\underline{M}=\left(X,\left(M_{x}\right)_{x \in X}\right)$ consisting of a set $X$ and a family $\left(M_{x}\right)_{x \in X}$ of $k$-vector spaces indexed by $X$. A morphism between $\underline{M}$ and $\underline{N}=\left(Y,\left(N_{y}\right)_{y \in Y}\right)$ is a couple $\underline{\varphi}=\left(f,\left(\varphi_{x}\right)_{x \in X}\right)$ with $f: X \rightarrow Y$ a function and $\left(\varphi_{x}: M_{x} \rightarrow N_{f(x)}\right)_{x \in X}$ a family of $k$-linear maps. If $\underline{\chi}=\left(g,\left(\chi_{y}\right)_{y \in Y}\right): \underline{N} \rightarrow \underline{P}=\left(Z,\left(P_{z}\right)_{z \in Z}\right)$ is another morphism in $\mathcal{Z}_{k}$ then $\underline{\chi} \circ \underline{\varphi}=\left(g \circ f,\left(\chi_{f(x)} \circ \varphi_{x}\right)_{x \in X}\right)$.

The category $\mathcal{Z}_{k}$ has a monoidal structure. The tensor product of $\underline{M}$ and $\underline{N}$ is given by

$$
\underline{M} \otimes \underline{N}=\left(X \times Y,\left(M_{x} \otimes N_{y}\right)_{(x, y) \in X \times Y}\right)
$$

and the unit object is $(\{*\}, k)$. The tensor product of two morphisms $\varphi: \underline{M} \rightarrow \underline{N}$, $\underline{\varphi^{\prime}}=\left(f^{\prime},\left(\varphi_{x^{\prime}}^{\prime}\right)_{x^{\prime} \in X^{\prime}}\right): \underline{M^{\prime}}=\left(X^{\prime},\left(M_{x^{\prime}}^{\prime}\right)_{x^{\prime} \in X^{\prime}}\right) \rightarrow \underline{N^{\prime}}=\left(Y^{\prime},\left(N_{y^{\prime}}^{\prime}\right)_{y^{\prime} \in Y^{\prime}}\right)$ in $\mathcal{Z}_{k}$ is defined by

$$
\underline{\varphi} \otimes \underline{\varphi^{\prime}}=\left(f \times f^{\prime},\left(\varphi_{x} \otimes \varphi_{x^{\prime}}^{\prime}\right)_{\left(x, x^{\prime}\right) \in X \times X^{\prime}}\right) .
$$

The natural isomorphisms $a, l, r$ of $\mathcal{Z}_{k}$ are obtained canonically from those of $\underline{\text { Sets }}$ and ${ }_{k} \mathcal{M}$, we leave the details to the reader. 
We claim that $\underline{1}=(\{*\}, k)$ is a left $\otimes$-generator for $\mathcal{Z}_{k}$. Indeed, let

$$
\underline{M} \otimes \underline{N}=\left(X \times Y,\left(M_{x} \otimes N_{y}\right)_{(x, y) \in X \times Y)} \underset{\underline{\chi}=\left(g,\left(\chi_{(x, y)}\right)_{(x, y) \in X \times Y}\right)}{\stackrel{\varphi=\left(f,\left(\varphi_{(x, y)}\right)_{(x, y) \in X \times Y}\right)}{\longrightarrow}} P=\left(Z,\left(P_{z}\right)_{z \in Z}\right)\right.
$$

be morphisms in $\mathcal{Z}_{k}$ such that $\varphi\left(\underline{\epsilon} \otimes \operatorname{Id}_{\underline{N}}\right)=\chi\left(\underline{\epsilon} \otimes \operatorname{Id}_{\underline{N}}\right)$, for all $\underline{\epsilon}=(\epsilon:\{*\} \rightarrow X$, $\left.\lambda: k \rightarrow M_{\epsilon(*)}\right)$ in $\mathcal{Z}_{k}$. On one hand, we have that $f\left(\epsilon \times \operatorname{Id}_{Y}\right)=g\left(\epsilon \times \operatorname{Id}_{Y}\right)$, for all $\epsilon:\{*\} \rightarrow X$ in Sets, and so $f=g$. On the other hand, for all $\epsilon:\{*\} \rightarrow X$ and $\lambda: k \rightarrow M_{\epsilon(*)}$ in ${ }_{k} \mathcal{M}$ we have $\varphi_{(\epsilon(*), y)}\left(\lambda \otimes I d_{N_{y}}\right)=\chi_{(\epsilon(*), y)}\left(\lambda \otimes I d_{N_{y}}\right)$, for all $y \in Y$. Hence, if we pick $x \in X$ and define $\epsilon_{x}:\{*\} \rightarrow X$ by $\epsilon_{x}(*)=x$ we then get $\varphi_{(x, y)}\left(\lambda \otimes I d_{N_{y}}\right)=\chi_{(x, y)}\left(\lambda \otimes I d_{N_{y}}\right)$, for all $\lambda: k \rightarrow M_{x}$ in ${ }_{k} \mathcal{M}$. It then follows that $\varphi_{(x, y)}=\chi_{(x, y)}$, for all $(x, y) \in X \times Y$, and therefore $\underline{\varphi}=\underline{\chi}$, as desired.

There are situations when a monoidal category $\mathcal{C}$ admits a left $\otimes$-generator that is different from the unit object.

6) Let $k$ be a field and $\mathcal{T}_{k}$ the category of Turaev over $k$, [11]. It has the same objects as $\mathcal{Z}_{k}$ but a different set of morphisms. More exactly, a morphism between two objects $\underline{M}=\left(X,\left(M_{x}\right)_{x \in X}\right)$ and $\underline{N}=\left(Y,\left(N_{y}\right)_{y \in Y}\right)$ is a pair $\varphi=\left(f,\left(\varphi_{y}\right)_{y \in Y}\right)$ consisting of a function $f: Y \rightarrow X$ and a family of $k$-linear maps $\left(\varphi_{y}: M_{f(y)} \rightarrow N_{y}\right)_{y \in Y}$. If $\underline{\chi}=\left(g,\left(\chi_{z}\right)_{z \in Z}\right): \underline{N} \rightarrow \underline{P}=\left(Z,\left(P_{z}\right)_{z \in Z}\right)$ is another morphism in $\mathcal{T}_{k}$ then $\chi \circ \underline{\varphi}=\left(f \circ g,\left(\chi_{z} \circ \varphi_{g(x)}\right)_{z \in Z}\right)$.

$\mathcal{T}_{k}$ is monoidal with tensor product defined by (3.3) and $\varphi \otimes \varphi^{\prime}=\left(f \times f^{\prime}\right.$,

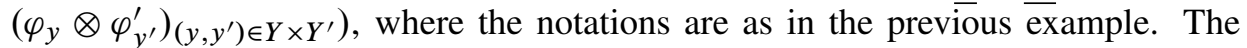
unit object is $(\{*\}, k)$ and $a, l, r$ are obtained naturally from those of Sets and ${ }_{k} \mathcal{M}$.

This time we claim that $(\{0,1\},(k, k))$ is a left $\otimes$-generator for $\mathcal{T}_{k}$. To this end consider

$$
\underline{M} \otimes \underline{N}=\left(X \times Y,\left(M_{x} \otimes N_{y}\right)_{(x, y) \in X \times Y)} \underset{\underline{\underline{\chi}=\left(g,\left(\chi_{z}\right)_{z \in Z}\right)}}{\stackrel{\varphi=\left(f,\left(\varphi_{z}\right)_{z \in Z}\right)}{\longrightarrow}} \underline{P}=\left(Z,\left(P_{z}\right)_{z \in Z}\right)\right.
$$

morphisms in $\mathcal{T}_{k}$ such that $\varphi\left(\underline{\epsilon} \otimes \operatorname{Id}_{N}\right)=\chi\left(\underline{\epsilon} \otimes \operatorname{Id}_{N}\right)$, for all $\underline{\epsilon}=(\epsilon: X \rightarrow\{0,1\}$, $\left.\left(\lambda_{x}: k=k_{\epsilon(x)} \rightarrow M_{x}\right)_{x \in X}\right)$ in $\mathcal{T}_{k}$. Firstly, this implies $\left(\epsilon \times \operatorname{Id}_{Y}\right) f=\left(\epsilon \times \operatorname{Id}_{Y}\right) g$, for all $\epsilon: X \rightarrow\{0,1\}$. If we denote $f(z)=\left(f_{1}(z), f_{2}(z)\right) \in X \times Y$, and adopt a similar notation for $g(z)$, we conclude that $\left(\epsilon f_{1}, f_{2}\right)=\left(\epsilon g_{1}, g_{2}\right)$, for all $\epsilon: X \rightarrow\{0,1\}$. Since $\{0,1\}$ is a cogenerator in Sets it follows that $f=g$. (A direct approach for the last assertion is the following: by the way of contradiction, if $f_{1} \neq g_{1}$ then there exists $z \in Z$ such that $f_{1}(z) \neq g_{1}(z)$; so if we consider $\epsilon: X \rightarrow\{0,1\}$ given by $\epsilon\left(f_{1}(z)\right)=0, \epsilon\left(g_{1}(z)\right)=1$ and arbitrary on the remaining elements of $X$ we then get that there is an $\epsilon: X \rightarrow\{0,1\}$ such that $\epsilon f_{1} \neq \epsilon f_{2}$, a contradiction.)

Secondly, if we denote $f(z)=g(z)=(u(z), v(z))$, for all $z \in Z$, then $\varphi_{z}\left(\lambda_{u(z)} \otimes \operatorname{Id}_{N_{v(z)}}\right)=\chi_{z}\left(\lambda_{u(z)} \otimes \operatorname{Id}_{N_{v(z)}}\right)$, for all $k$-linear maps $\lambda_{u(z)}: k \rightarrow M_{u(z)}$. It is immediate that this implies $\varphi_{z}=\chi_{z}$, for all $z \in Z$, so we are done. 
7) Let $G$ be a group and let $k$ be a field. If we denote by $\operatorname{Vect}^{G}$ the category of $G$-graded $k$-vector spaces and $k$-linear morphisms that preserve the gradings then $\mathrm{Vect}^{G}$ is a monoidal category. For

$$
V=\bigoplus_{g \in G} V_{g} \text { and } W=\bigoplus_{g \in G} W_{g}
$$

two $G$-graded $k$-vector spaces their tensor product is the same as in ${ }_{k} \mathcal{M}$, and it is considered as a $G$-graded vector space via the grading given by

$$
(V \otimes W)_{g}=\bigoplus_{\sigma \tau=g} V_{\sigma} W_{\tau},
$$

for all $g \in G$. On morphisms the tensor product in $\mathrm{Vect}^{G}$ is defined exactly as in ${ }_{k} \mathcal{M}$. The unit object is $k$ viewed as a $G$-graded vector space in the trivial way: $k_{e}=k$ and $k_{g}=0$, for all $G \ni g \neq e$, where $e$ is the neutral element of $G$. The remarkable fact is that for $\operatorname{Vect}^{G}$ the associativity constraints $a$ are in a one to one correspondence with the normalized 3-cocycles $\phi$ on $G$, while the left and right unit constraints are always equal with those of ${ }_{k} \mathcal{M}$. This monoidal structure on Vect ${ }^{G}$ will be denoted by $\operatorname{Vect}_{\phi}^{G}$.

It is left to the reader to show that the group algebra of $G$,

$$
k[G]=\bigoplus_{g \in G} k g,
$$

is a left $\otimes$-generator for $\operatorname{Vect}_{\phi}^{G}$, for any normalized 3-cocycle $\phi$ on $G$. Mostly this is a consequence of the fact that any element $v$ of a $G$-graded vector space $V$ gives rise to a morphism from $k[G]$ into $V$ in $\operatorname{Vect}_{\phi}^{G}$, and vice-versa.

8) Let $H$ be a bialgebra over a field $k$ and ${ }_{H} \mathcal{M}$ the category of its left representations. It is a classical result in the Hopf algebra theory that the monoidal structure of ${ }_{k} \mathcal{M}$ induces a monoidal structure on ${ }_{H} \mathcal{M}$ : the $H$-module structure on a tensor product is given by the comultiplication of $H$ and the unit object is $k$ regarded as a left $H$-module via the counit of $H$.

Consider $H$ as an object in ${ }_{H} \mathcal{M}$ via its multiplication. It is immediate that a morphism $H \rightarrow M$ in ${ }_{H} \mathcal{M}$ is completely determined by an element $m \in M$, so $H$ is a left $\otimes$-generator in $H \mathcal{M}$.

9) Let $k$ be a field and $H$ a weak bialgebra over $k$ with unit 1, comultiplication $\Delta(h)=h_{1} \otimes h_{2}, h \in H$, and counit $\varepsilon_{H}$. (For the complete set of axioms we invite the reader to consult [2].)

The category of left $H$-representations, ${ }_{H} \mathcal{M}$, is monoidal. This time the tensor product is the tensor product over the $k$-algebra $H_{t}:=\left\{h \in H \mid \Delta(h)=h \mathbf{1}_{1} \otimes \mathbf{1}_{2}\right\}$, the target subalgebra of $H$. Note that, if $M$ is a left $H$-module then it is a left $H_{t}$-module via the functor restriction of scalars defined by the embedding 
$H_{t} \hookrightarrow H$ and, moreover, an $H_{t}$-bimodule via the right $H_{t}$-module structure defined by $m \cdot r=\bar{\varepsilon}_{s}(r) \cdot m$, for all $m \in M, r \in H_{t}$. Here $\bar{\varepsilon}_{s}: H \rightarrow H$ is given by $\bar{\varepsilon}_{s}(h)=\varepsilon_{H}\left(\mathbf{1}_{2} h\right) \mathbf{1}_{1}$, for all $h \in H$; we should remark that $\left.\bar{\varepsilon}_{s}\right|_{H_{t}}$ is an anti-algebra morphism.

The $H$-module structure on a tensor product $M \otimes_{H_{t}} N$ is defined via the comultiplication of $H$. The unit is $H_{t}$ considered as a left $H$-module via the action $h \triangleright r=\varepsilon_{t}(h r)=\varepsilon_{t}\left(h \bar{\varepsilon}_{s}(r)\right)$, for all $h \in H, r \in H_{t}$, where $\varepsilon_{t}: H \rightarrow H$ sends $h \in H$ to $\varepsilon_{H}\left(\mathbf{1}_{1} h\right) \mathbf{1}_{2}$. It is well defined since $\operatorname{Im}\left(\varepsilon_{t}\right)=H_{t}$.

One can see easily that $H$, regarded as a left $H$-module via its multiplication, is a left $\otimes_{H_{t}}$-generator for $H \mathcal{M}$. We should also remark that the $k$-algebra $H_{t}$ is separable (see for instance [52, Proposition 1.6]). So the category of $H_{t}$-bimodules produces a concrete example of monoidal category having the unit object a left $\otimes$ generator.

For simplicity, throughout this section we assume that $\mathcal{C}$ is a monoidal category with coequalizers and such that any object of it is coflat.

Lemma 3.3. Assume that 1 is a left $\otimes$-generator for $\mathcal{C}$ and let $i: R \rightarrow S$ be an algebra morphism, $F: \mathcal{C}_{S} \rightarrow \mathcal{C}_{R}$ the restriction of scalars functor induced by $i$ and $-\otimes_{R} S: \mathcal{C}_{R} \rightarrow \mathcal{C}_{S}$ the induction functor, respectively. Then there exists an isomorphism

$$
\operatorname{Nat}\left(F \circ\left(-\otimes_{R} S\right),-\right) \cong{ }_{R} \operatorname{Hom}_{R}(S, R),
$$

where ${ }_{R} \operatorname{Hom}_{R}(S, R)$ stands for the set of $R$-bimodule morphisms from $S$ to $R$ in $\mathcal{C}$.

Proof. Follows the same line as the proof of [10, Theorem 2.7, 3.]. We first show that the desired isomorphism is produced by

$$
\alpha: \operatorname{Nat}\left(F \circ\left(-\otimes_{R} S\right),-\right) \cong{ }_{R} \operatorname{Hom}_{R}(S, R),
$$

defined as follows. If $u=\left(u_{X}: X \otimes_{R} S \rightarrow X\right)_{X \in \mathcal{C}_{R}}$ is in $\operatorname{Nat}\left(F \circ\left(-\otimes_{R} S\right),-\right)$ we then define $\alpha(u)=u_{R} \Upsilon_{S}^{\prime-1}$. To show that $\alpha(u)$ is a right $R$-linear morphism is sufficient to show that $\Upsilon_{S}^{\prime-1}$ is so. Indeed, on one hand we have

$$
\begin{aligned}
\mu_{R \otimes_{R} S}^{R}\left(\operatorname{Id}_{R} \otimes \Upsilon_{S}^{\prime-1}\right) & =\mu_{R \otimes_{R} S}^{R}\left(\operatorname{Id}_{R} \otimes q_{R, S}^{R}\left(\underline{\eta}_{R} \otimes \operatorname{Id}_{S}\right)\right) \\
& =q_{R, S}^{R}\left(\underline{m}_{R}\left(\operatorname{Id}_{R} \otimes \underline{\eta}_{R}\right) \otimes \operatorname{Id}_{S}\right)=q_{R, S}^{R} .
\end{aligned}
$$

On the other hand,

$$
\begin{aligned}
\Upsilon_{S}^{\prime-1} \mu_{S}^{R} & =\Upsilon_{S}^{\prime-1} \underline{m}_{S}\left(i \otimes \operatorname{Id}_{S}\right) \\
& =q_{R, S}^{R}\left(\underline{\eta}_{R} \otimes \operatorname{Id}_{S}\right) \underline{m}_{S}\left(i \otimes \operatorname{Id}_{S}\right) \\
& =q_{R, S}^{R}\left(\underline{m}_{R}\left(\underline{\eta}_{R} \otimes \operatorname{Id}_{R}\right) \otimes \operatorname{Id}_{S}\right)=q_{R, S}^{R},
\end{aligned}
$$

as required. 
Take $\epsilon: \underline{1} \rightarrow R$ an arbitrary morphism in $\mathcal{C}$ and define $f_{\epsilon}: R \rightarrow R$ by $f_{\varepsilon}=\underline{m}_{R}\left(\epsilon \otimes \operatorname{Id}_{R}\right)$. Since $\underline{m}_{R}$ is associative we get that $f_{\epsilon}$ is a morphism in $\mathcal{C}_{R}$, and therefore the naturality of $u$ implies that $f_{\epsilon} u_{R}=u_{R} \widehat{f}_{\epsilon}$. This is equivalent to $f_{\epsilon} u_{R} \Upsilon_{S}^{\prime-1}=u_{R} \widehat{f}_{\epsilon} \Upsilon_{S}^{\prime-1}$, and so with

$$
\underline{m}_{R}\left(\operatorname{Id}_{R} \otimes \alpha(u)\right)\left(\epsilon \otimes \operatorname{Id}_{S}\right)=u_{R} q_{R, S}^{R}\left(\epsilon \otimes \operatorname{Id}_{S}\right) .
$$

Since

$$
\begin{aligned}
\alpha(u) \underline{m}_{S}\left(i \otimes \operatorname{Id}_{S}\right) & =u_{R} \Upsilon_{S}^{\prime-1} \underline{m}_{S}\left(i \otimes \operatorname{Id}_{S}\right) \\
& =u_{R} q_{R, S}^{R}\left(\underline{\eta}_{R} \otimes \operatorname{Id}_{S}\right) \underline{m}_{S}\left(i \otimes \operatorname{Id}_{S}\right) \\
& =u_{R} q_{R, S}^{R}\left(\operatorname{Id}_{R} \otimes \underline{m}_{S}\left(i \otimes \operatorname{Id}_{S}\right)\right)\left(\underline{\eta}_{R} \otimes \operatorname{Id}_{R \otimes S}\right) \\
& =u_{R} q_{R, S}^{R}\left(\underline{m}_{R}\left(\underline{\eta}_{R} \otimes \operatorname{Id}_{R}\right) \otimes \operatorname{Id}_{S}\right)=u_{R} q_{R, S}^{R},
\end{aligned}
$$

where in the next-to-last equality we used that $\left(q_{R, S}^{R}, R \otimes_{R} S\right)$ is a coequalizer, we obtain that

$$
\underline{m}_{R}\left(\operatorname{Id}_{R} \otimes \alpha(u)\right)\left(\epsilon \otimes \operatorname{Id}_{S}\right)=\alpha(u) \underline{m}_{S}\left(i \otimes \operatorname{Id}_{S}\right)\left(\epsilon \otimes \operatorname{Id}_{S}\right),
$$

for all $\epsilon: \underline{1} \rightarrow R$. The fact that $\underline{1}$ is a left $\otimes$-generator for $\mathcal{C}$ implies now that $\alpha(u)$ is left $R$-linear as well, and thus an $R$-bimodule morphism in $\mathcal{C}$.

We claim that $\alpha$ is an isomorphism with inverse defined by

$$
\alpha^{-1}(\vartheta)=\left(v_{X}=\Upsilon_{X} \tilde{\vartheta}: X \otimes_{R} S \rightarrow X\right)_{X \in \mathcal{C}_{R}}, \vartheta \in{ }_{R} \operatorname{Hom}_{R}(S, R) .
$$

One can easily see that $v=\left(v_{X}\right)_{X \in \mathcal{C}_{R}}$ is completely determined by the property $v_{X} q_{X, S}^{R}=v_{X}^{R}\left(\operatorname{Id}_{X} \otimes \vartheta\right)$, for any $X \in \mathcal{C}_{R}$, and this allows us to prove easily that $v$ is indeed a natural transformation. Thus $\alpha^{-1}$ is well defined. Furthermore, $\alpha^{-1} \alpha(u)=$ $\alpha^{-1}\left(u_{R} \Upsilon_{S}^{\prime-1}\right)=\left(v_{X}=\Upsilon_{X} u_{R} \Upsilon_{S}^{\prime-1}\right)_{X \in \mathcal{C}_{R}}$ with $v_{X}$ characterized by the fact that $v_{X} q_{X, S}^{R}=v_{X}^{R}\left(\operatorname{Id}_{X} \otimes \alpha(u)\right)$.

Take now $\epsilon: \underline{1} \rightarrow X$ an arbitrary morphism in $\mathcal{C}$ and define $g_{\epsilon}=v_{X}^{R}\left(\epsilon \otimes \operatorname{Id}_{R}\right)$ : $R \rightarrow X$. It is immediate that $g_{\epsilon}$ is right $R$-linear, hence by the naturality of $u$ we have

$g_{\epsilon} \alpha(u)=g_{\epsilon} u_{R} \Upsilon_{S}^{\prime-1}=u_{X} \widehat{g_{\epsilon}} \Upsilon_{S}^{\prime-1}=u_{X} q_{X, S}^{R}\left(g_{\epsilon} \underline{\eta}_{R} \otimes \operatorname{Id}_{S}\right)=u_{X} q_{X, S}^{R}\left(\epsilon \otimes \operatorname{Id}_{S}\right)$.

But $g_{\epsilon} \alpha(u)=v_{X}^{R}\left(\epsilon \otimes \operatorname{Id}_{R}\right) \alpha(u)=v_{X}^{R}\left(\operatorname{Id}_{X} \otimes \alpha(u)\right)\left(\epsilon \otimes \operatorname{Id}_{S}\right)$, so using again that $\underline{1}$ is a left $\otimes$-generator in $\mathcal{C}$ we deduce that $v_{X}^{R}\left(\operatorname{Id}_{X} \otimes \alpha(u)\right)=u_{X} q_{X, S}^{R}$, for all $X$ in $\overline{\mathcal{C}}$. This implies $v_{X} q_{X, S}^{R}=u_{X} q_{X, S}^{R}$ and since $q_{X, S}^{R}$ is an epimorphism in $\mathcal{C}$ we conclude that $v_{X}=u_{X}$, for all $X$ in $\mathcal{C}$, and thus $\alpha^{-1} \alpha(u)=u$. 
For $\vartheta \in{ }_{R} \operatorname{Hom}_{R}(S, R)$ we have

$$
\begin{aligned}
\alpha \alpha^{-1}(\vartheta) & =\alpha\left(\left(v_{X}=\Upsilon_{X} \tilde{\vartheta}: X \otimes_{R} S \rightarrow X\right)_{X \in \mathcal{C}_{R}}\right) \\
& =v_{R} \Upsilon_{S}^{\prime-1}=v_{R} q_{R, S}^{R}\left(\underline{\eta}_{R} \otimes \operatorname{Id}_{S}\right)=v_{R}^{R}\left(\operatorname{Id}_{R} \otimes \vartheta\right)\left(\underline{\eta}_{R} \otimes \operatorname{Id}_{S}\right)=\vartheta .
\end{aligned}
$$

Thus $\alpha$ and $\alpha^{-1}$ are inverses, as claimed.

Lemma 3.4. Under the same hypothesis as in Lemma 3.3 we have that

$$
\begin{aligned}
& \beta: \operatorname{Nat}\left(-,\left(-\otimes_{R} S\right) \circ F\right) \rightarrow \mathcal{W} \\
& \quad:=\left\{e: \underline{1} \rightarrow S \otimes_{R} S \mid \mu_{S \otimes_{R} S}^{S}\left(\operatorname{Id}_{S} \otimes e\right)=v_{S \otimes_{R} S}^{S}\left(e \otimes \operatorname{Id}_{S}\right)\right\}
\end{aligned}
$$

given by $\beta(\zeta)=\zeta_{S} \underline{\eta}_{S}$ is well defined and an isomorphism.

Proof. Similar to the one of Lemma 3.3. First, observe that since $\zeta_{S}$ is right $S$-linear we have

$$
v_{S \otimes_{R} S}^{S}\left(\beta(\zeta) \otimes \operatorname{Id}_{S}\right)=v_{S \otimes_{R} S}^{S}\left(\zeta_{S} \otimes \operatorname{Id}_{S}\right)\left(\underline{\eta}_{S} \otimes \operatorname{Id}_{S}\right)=\zeta_{S} \underline{m}_{S}\left(\underline{\eta}_{S} \otimes \operatorname{Id}_{S}\right)=\zeta_{S} .
$$

Let $\epsilon: \underline{1} \rightarrow S$ be an arbitrary morphism in $\mathcal{C}$ and define $h_{\epsilon}=\underline{m}_{S}\left(\epsilon \otimes \operatorname{Id}_{S}\right): S \rightarrow S$. Clearly $h_{\epsilon}$ is right $S$-linear, so by the naturality of $\zeta$ we deduce that $\widehat{h_{\epsilon}} \zeta_{S}=\zeta_{S} h_{\epsilon}$. A direct computation ensures us that

$$
{\widehat{h_{\epsilon}}}_{q_{S, S}^{R}}^{R}=q_{S, S}^{R}\left(\underline{m}_{S}\left(\epsilon \otimes \operatorname{Id}_{S}\right) \otimes \operatorname{Id}_{S}\right)=\mu_{S \otimes_{R} S}^{S}\left(\epsilon \otimes \operatorname{Id}_{S \otimes_{R} S}\right) q_{S, S}^{R},
$$

thus $\widehat{h_{\epsilon}}=\mu_{S \otimes_{R} S}^{S}\left(\epsilon \otimes \operatorname{Id}_{S \otimes_{R} S}\right)$. From here we obtain

$$
\zeta_{S} \underline{m}_{S}\left(\epsilon \otimes \operatorname{Id}_{S}\right)=\zeta_{S} h_{\epsilon}=\widehat{h_{\epsilon}} \zeta_{S}=\mu_{S \otimes_{R} S}^{S}\left(\operatorname{Id}_{S} \otimes \zeta_{S}\right)\left(\epsilon \otimes \operatorname{Id}_{S}\right),
$$

for all $\epsilon: \underline{1} \rightarrow S$ in $\mathcal{C}$. Using again that $\underline{1}$ is a left $\otimes$-generator in $\mathcal{C}$ we conclude that $\zeta_{S}$ is also left $S$-linear, and hence

$$
\begin{aligned}
\mu_{S \otimes_{R} S}^{S}\left(\operatorname{Id}_{S} \otimes \beta(\zeta)\right) & =\mu_{S \otimes_{R} S}^{S}\left(\operatorname{Id}_{S} \otimes \zeta_{S}\right)\left(\operatorname{Id}_{S} \otimes \underline{\eta}_{S}\right) \\
& =\zeta_{S} \underline{m}_{S}\left(\operatorname{Id}_{S} \otimes \underline{\eta}_{S}\right) \\
& =\zeta_{S} \\
& =v_{S \otimes_{R} S}^{S}\left(\beta(\zeta) \otimes \operatorname{Id}_{S}\right),
\end{aligned}
$$

proving that $\beta$ is well defined. We assert now that $\beta$ is an isomorphism. To construct its inverse we proceed as follows. If $\mathfrak{M}$ is a right $S$-module then

$$
\mathfrak{M} \otimes S \otimes R \otimes S \stackrel{\operatorname{Id}_{\mathfrak{M} \otimes S} \otimes \underline{m}_{S}\left(i \otimes \mathrm{Id}_{S}\right)}{\longrightarrow} \mathfrak{M} \otimes S \otimes S \stackrel{\operatorname{Id}_{\mathfrak{M}} \otimes q_{S}^{R}, S}{\longrightarrow} \mathfrak{M} \otimes S \otimes_{R} S
$$

is a coequalizer in $\mathcal{C}$. Furthermore, the morphism $q_{\mathfrak{M}, S}^{R}\left(\nu_{\mathfrak{M}}^{S} \otimes \operatorname{Id}_{S}\right)$ fits in the universal property of this coequalizer, and so there is a unique morphism $\bar{\zeta}_{\mathfrak{M}}: \mathfrak{M} \otimes S \otimes_{R} S \rightarrow \mathfrak{M} \otimes_{R} S$ such that $\bar{\zeta}_{\mathfrak{M}}\left(\operatorname{Id}_{\mathfrak{M}} \otimes q_{S, S}^{R}\right)=q_{\mathfrak{M}, S}^{R}\left(\nu_{\mathfrak{M}}^{S} \otimes \operatorname{Id}_{S}\right)$. 
We claim that $\beta^{-1}: \mathcal{W} \rightarrow \operatorname{Nat}\left(-,\left(-\otimes_{R} S\right) \circ F\right)$ given by

$$
\beta^{-1}(e)=\left(\zeta_{\mathfrak{M}}^{e}=\bar{\zeta}_{\mathfrak{M}}\left(\operatorname{Id}_{\mathfrak{M}} \otimes e\right): \mathfrak{M} \rightarrow \mathfrak{M} \otimes_{R} S\right)_{\mathfrak{M} \in \mathcal{C}_{S}}
$$

is well defined and the inverse of $\beta$. To see that $\zeta_{\mathfrak{M}}^{e}$ is right $S$-linear observe that

$$
\begin{aligned}
\bar{\zeta}_{\mathfrak{M}}\left(v_{\mathfrak{M}}^{S} \otimes \operatorname{Id}_{S \otimes_{R} S}\right)\left(\operatorname{Id}_{\mathfrak{M} \otimes S} \otimes q_{S, S}^{R}\right) & =\bar{\zeta}_{\mathfrak{M}}\left(\operatorname{Id}_{\mathfrak{M}} \otimes q_{S, S}^{R}\right)\left(v_{\mathfrak{M}}^{S} \otimes \operatorname{Id}_{S \otimes S}\right) \\
& =q_{\mathfrak{M}, S}^{R}\left(v_{\mathfrak{M}}^{S}\left(v_{\mathfrak{M}}^{S} \otimes \operatorname{Id}_{S}\right) \otimes \operatorname{Id}_{S}\right) \\
& =q_{\mathfrak{M}, S}^{R}\left(v_{\mathfrak{M}}^{S} \otimes \operatorname{Id}_{S}\right)\left(\operatorname{Id}_{\mathfrak{M}} \otimes \underline{m}_{S} \otimes \operatorname{Id}_{S}\right) \\
& =\bar{\zeta}_{\mathfrak{M}}\left(\operatorname{Id}_{\mathfrak{M}} \otimes \mu_{S \otimes_{R} S}^{S}\right)\left(\operatorname{Id}_{\mathfrak{M} \otimes S} \otimes q_{S, S}^{R}\right)
\end{aligned}
$$

and since $\operatorname{Id}_{\mathfrak{M} \otimes S} \otimes q_{S, S}^{R}$ is an epimorphism this shows that $\bar{\zeta}_{\mathfrak{M}}\left(v_{\mathfrak{M}}^{S} \otimes \operatorname{Id}_{S \otimes_{R} S}\right)=$ $\bar{\zeta}_{\mathfrak{M}}\left(\operatorname{Id}_{\mathfrak{M}} \otimes \mu_{S \otimes_{R} S}^{S}\right)$. The latter equality allows us to compute

$$
\begin{aligned}
\zeta_{\mathfrak{M}}^{e} \nu_{\mathfrak{M}}^{S} & =\bar{\zeta}_{\mathfrak{M}}\left(\operatorname{Id}_{\mathfrak{M}} \otimes e\right) \nu_{\mathfrak{M}}^{S}=\bar{\zeta}_{\mathfrak{M}}\left(v_{\mathfrak{M}}^{S} \otimes \operatorname{Id}_{S \otimes_{R} S}\right)\left(\operatorname{Id}_{\mathfrak{M} \otimes S} \otimes e\right) \\
& =\bar{\zeta}_{\mathfrak{M}}\left(\operatorname{Id}_{\mathfrak{M}} \otimes \mu_{S \otimes_{R} S}^{S}\left(\operatorname{Id}_{S} \otimes e\right)\right)=\bar{\zeta}_{\mathfrak{M}}\left(\operatorname{Id}_{\mathfrak{M}} \otimes v_{S \otimes_{R} S}^{S}\left(e \otimes \operatorname{Id}_{S}\right)\right) .
\end{aligned}
$$

Similar arguments leads us to

$v_{\mathfrak{M} \otimes_{R} S}^{S}\left(\bar{\zeta}_{\mathfrak{M}} \otimes \operatorname{Id}_{S}\right)\left(\operatorname{Id}_{\mathfrak{M}} \otimes q_{S, S}^{R} \otimes \operatorname{Id}_{S}\right)=\bar{\zeta}_{\mathfrak{M}}\left(\operatorname{Id}_{\mathfrak{M}} \otimes v_{S \otimes_{R} S}^{S}\right)\left(\operatorname{Id}_{\mathfrak{M}} \otimes q_{S, S}^{R} \otimes \operatorname{Id}_{S}\right)$,

and this allows us to deduce that $v_{\mathfrak{M} \otimes_{R} S}^{S}\left(\bar{\zeta}_{\mathfrak{M}} \otimes \operatorname{Id}_{S}\right)=\bar{\zeta}_{\mathfrak{M}}\left(\operatorname{Id}_{\mathfrak{M}} \otimes v_{S \otimes_{R} S}^{S}\right)$. From here it is immediate that

$$
v_{\mathfrak{M} \otimes_{R} S}^{S}\left(\zeta_{\mathfrak{M}}^{e} \otimes \operatorname{Id}_{S}\right)=\bar{\zeta}_{\mathfrak{M}}\left(\operatorname{Id}_{\mathfrak{M}} \otimes v_{S \otimes_{R} S}^{S}\left(e \otimes \operatorname{Id}_{S}\right)\right),
$$

and so $\zeta_{\mathfrak{M}}^{e}$ is right $S$-linear, as desired. Next, if $f: \mathfrak{M} \rightarrow \mathfrak{N}$ is a morphism in $\mathcal{C}_{S}$ then by arguments similar to the ones above we get that $\widehat{f} \bar{\zeta}_{\mathfrak{M}}=\bar{\zeta}_{\mathfrak{N}}\left(f \otimes \operatorname{Id}_{S \otimes_{R} S}\right)$. Thus $\hat{f} \zeta_{\mathfrak{M}}^{e}=\bar{\zeta}_{\mathfrak{N}}\left(f \otimes \operatorname{Id}_{S \otimes_{R} S}\right)\left(\operatorname{Id}_{\mathfrak{M}} \otimes e\right)=\bar{\zeta}_{\mathfrak{N}}\left(\operatorname{Id}_{\mathfrak{N}} \otimes e\right) f=\zeta_{\mathfrak{N}}^{e} f$, and this ends the fact that $\beta^{-1}$ is well defined.

Let now $\zeta \in \operatorname{Nat}\left(-,\left(-\otimes_{R} S\right) \circ F\right)$ and $e=\beta^{-1}(\zeta)$, that is $e=\zeta_{S} \underline{\eta}_{S}$. If $\mathfrak{M}$ is a right $S$-module and $\epsilon: \underline{1} \rightarrow \mathfrak{M}$ an arbitrary morphism then $\hbar_{\epsilon}=v_{\mathfrak{M}}^{S}\left(\epsilon \otimes \operatorname{Id}_{S}\right)$ : $S \rightarrow \mathfrak{M}$ is right $S$-linear. By the naturality of $\zeta$ we obtain that $\zeta_{\mathfrak{M}} \hbar_{\epsilon}=\widehat{\hbar_{\epsilon}} \zeta_{S}$. Together with

$\widehat{\hbar_{\epsilon}} q_{S, S}^{R}=q_{\mathfrak{M}, S}^{R}\left(\hbar_{\epsilon} \otimes \operatorname{Id}_{S}\right)=q_{\mathfrak{M}, S}^{R}\left(\nu_{\mathfrak{M}}^{S}\left(\epsilon \otimes \operatorname{Id}_{S}\right) \otimes \operatorname{Id}_{S}\right)=\bar{\zeta}_{\mathfrak{M}}\left(\epsilon \otimes \operatorname{Id}_{S \otimes_{R} S}\right) q_{S, S}^{R}$, this implies that

$$
\zeta_{\mathfrak{M}} \epsilon=\zeta_{\mathfrak{M}} \hbar_{\epsilon} \underline{\eta}_{S}=\widehat{\hbar_{\epsilon}} \zeta_{S} \underline{\eta}_{S}=\bar{\zeta}_{\mathfrak{M}}\left(\epsilon \otimes \operatorname{Id}_{S \otimes_{R} S}\right) e=\bar{\zeta}_{\mathfrak{M}}\left(\operatorname{Id}_{\mathfrak{M}} \otimes e\right) \epsilon=\zeta_{\mathfrak{M}}^{e} \epsilon .
$$


By the assumption that 1 is a left $\otimes$-generator for $\mathcal{C}$ we conclude that $\zeta=\zeta^{e}$ or, otherwise stated, $\beta^{-1} \beta(\bar{\zeta})=\zeta$. $\beta^{-1}$ is also a right inverse for $\beta$ since for any $e \in \mathcal{W}$ we have

$$
\beta \beta^{-1}(e)=\beta\left(\zeta^{e}\right)=\zeta_{S}^{e} \underline{\eta}_{S}=\bar{\zeta}_{S}\left(\underline{\eta}_{S} \otimes \operatorname{Id}_{S \otimes_{R} S}\right) e=e,
$$

the last equality being a consequence of the following computation

$$
\begin{aligned}
\bar{\zeta}_{S}\left(\underline{\eta}_{S} \otimes \operatorname{Id}_{S \otimes_{R} S}\right) q_{S, S}^{R} & =\bar{\zeta}_{S}\left(\operatorname{Id}_{S} \otimes q_{S, S}^{R}\right)\left(\underline{\eta}_{S} \otimes \operatorname{Id}_{S \otimes S}\right) \\
& =q_{S, S}^{R}\left(v_{S}^{S}\left(\underline{\eta}_{S} \otimes \operatorname{Id}_{S}\right) \otimes \operatorname{Id}_{S}\right)=q_{S, S}^{R} .
\end{aligned}
$$

So our proof is finished.

One can prove now one of the main results of this section.

Theorem 3.5. Let $\mathcal{C}$ be a monoidal category having the property that its unit object 1 is a left $\otimes$-generator. If $i: R \rightarrow S$ is an algebra morphism in $\mathcal{C}$ then the following assertions are equivalent:

(i) The functor restriction of scalars $F: \mathcal{C}_{S} \rightarrow \mathcal{C}_{R}$ is a Frobenius functor;

(ii) There exist an R-bimodule morphism $\vartheta: S \rightarrow R$ and a morphism e $: \underline{1} \rightarrow$ $S \otimes_{R} S$ in $\mathcal{C}$ such that the following diagrams are commutative

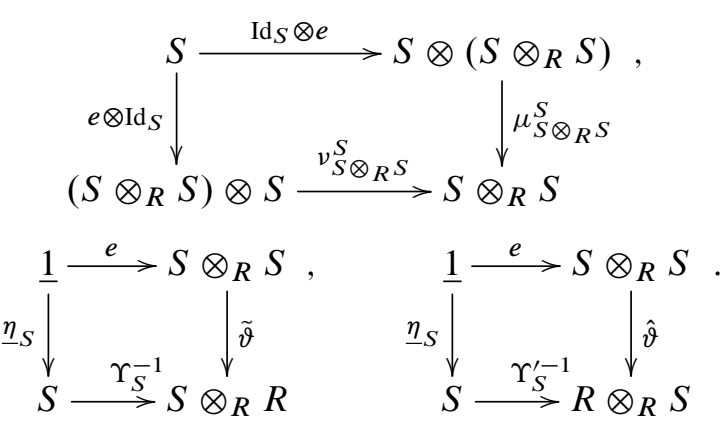

Proof. From the comments made before Definition 3.1 we have that $F$ is a Frobenius functor if and only if $-\otimes_{R} S: \mathcal{C}_{R} \rightarrow \mathcal{C}_{S}$ is a right adjoint for $F$, and from the previous two lemmas we have that the functor $-\otimes_{R} S$ is a right adjoint for $F$ if and only if there exist $\vartheta \in{ }_{R} \operatorname{Hom}_{R}(S, R)$ and $e \in \mathcal{W}$ such that

$$
v_{F(\mathfrak{M})} F\left(\zeta_{\mathfrak{M}}\right)=\operatorname{Id}_{\mathfrak{M}}, \forall \mathfrak{M} \in \mathcal{C}_{S} \text { and } \widehat{v_{X}} \zeta_{X \otimes_{R} S}=\operatorname{Id}_{X \otimes_{R} S}, \forall X \in \mathcal{C}_{R} \text {, }
$$

where

$$
v=\alpha^{-1}(\vartheta)=\left(v_{X}=\Upsilon_{X} \tilde{\vartheta}: X \otimes_{R} S \rightarrow X\right)_{X \in \mathcal{C}_{R}}
$$

in $\operatorname{Nat}\left(F \circ\left(-\otimes_{R} S\right),-\right)$, and

$$
\zeta:=\beta^{-1}(e)=\left(\zeta_{\mathfrak{M}}^{e}=\bar{\zeta}_{\mathfrak{M}}\left(\operatorname{Id}_{\mathfrak{M}} \otimes e\right): \mathfrak{M} \rightarrow \mathfrak{M} \otimes_{R} S\right)_{\mathfrak{M} \in \mathcal{C}_{S}}
$$

in $\operatorname{Nat}\left(-,\left(-\otimes_{R} S\right) \circ F\right)$ are the natural transformations defined by $\vartheta$ and $e$, respectively. 
We next prove that the first equality in (3.4) is equivalent to the fact that the second diagram in (ii) is commutative. That the second equality above is equivalent to the fact that the last diagram in (ii) is commutative can be proved in a similar way, the details are left to the reader.

Let us start by noting that $v_{F(\mathfrak{M})} F\left(\zeta_{\mathfrak{M}}\right)=\operatorname{Id}_{\mathfrak{M}}$ for all $\mathfrak{M} \in \mathcal{C}_{S}$ is equivalent to $\Upsilon_{\mathfrak{M}} \tilde{\vartheta} \bar{\zeta}_{\mathfrak{M}}\left(\mathrm{Id}_{\mathfrak{M}} \otimes e\right)=\mathrm{Id}_{\mathfrak{M}}$, for all $\mathfrak{M} \in \mathcal{C}_{S}$, and since $\Upsilon_{\mathfrak{M}}$ is an isomorphism the latter is equivalent to $\tilde{\vartheta} \bar{\zeta}_{\mathfrak{M}}\left(\operatorname{Id}_{\mathfrak{M}} \otimes e\right) \Upsilon_{\mathfrak{M}}=\operatorname{Id}_{\mathfrak{M} \otimes_{R} R}$ or, equivalently, to $\tilde{\vartheta} \bar{\zeta}_{\mathfrak{M}}\left(\operatorname{Id}_{\mathfrak{M}} \otimes e\right) v_{\mathfrak{M}}^{R}=q_{\mathfrak{M}, R}^{R}$, for all $\mathfrak{M} \in \mathcal{C}_{S}$. It is immediate that all these equivalent conditions are also equivalent to

$$
\tilde{\vartheta} \bar{\zeta}_{\mathfrak{M}}\left(\operatorname{Id}_{\mathfrak{M}} \otimes e\right)\left(v_{\mathfrak{M}}^{S}\left(\operatorname{Id}_{\mathfrak{M}} \otimes i\right) \otimes \operatorname{Id}_{S \otimes_{R} S}\right)\left(\operatorname{Id}_{\mathfrak{M} \otimes R} \otimes e\right)=q_{\mathfrak{M}, R}^{R}, \quad \forall \mathfrak{M} \in \mathcal{C}_{S}
$$

We claim that (3.5) holds if and only if $\Upsilon_{S} \tilde{\vartheta} e=\underline{\eta}_{S}$. Indeed, observe first that

$$
\begin{aligned}
\tilde{\vartheta} \bar{\zeta}_{\mathfrak{M}}\left(v_{\mathfrak{M}}^{S} \otimes \operatorname{Id}_{S \otimes_{R} S}\right)\left(\operatorname{Id}_{\mathfrak{M} \otimes S} \otimes q_{S, S}^{R}\right) & =q_{\mathfrak{M}, R}^{R}\left(v_{\mathfrak{M}}^{S}\left(\operatorname{Id}_{\mathfrak{M}} \otimes \underline{m}_{S}\right) \otimes \vartheta\right) \\
& =\tilde{\vartheta} \bar{\zeta}_{\mathfrak{M}}\left(\operatorname{Id}_{\mathfrak{M}} \otimes \bar{\zeta}_{S}\right)\left(\operatorname{Id}_{\mathfrak{M} \otimes S} \otimes q_{S, S}^{R}\right),
\end{aligned}
$$

and this implies $\tilde{\vartheta} \bar{\zeta}_{\mathfrak{M}}\left(v_{\mathfrak{M}}^{S} \otimes \operatorname{Id}_{S \otimes_{R} S}\right)=\tilde{\vartheta} \bar{\zeta}_{\mathfrak{M}}\left(\operatorname{Id}_{\mathfrak{M}} \otimes \bar{\zeta}_{S}\right)$. Therefore, (3.5) is equivalent to

$$
\tilde{\vartheta} \bar{\zeta}_{\mathfrak{M}}\left(\operatorname{Id}_{\mathfrak{M}} \otimes \bar{\zeta}_{S}\left(\operatorname{Id}_{S} \otimes e\right) i\right)=q_{\mathfrak{M}, R}^{R}, \quad \forall \mathfrak{M} \in \mathcal{C}_{S}
$$

One can check easily that

$$
\Upsilon_{\mathfrak{M}} \tilde{\vartheta} \bar{\zeta}_{\mathfrak{M}}\left(\operatorname{Id}_{\mathfrak{M}} \otimes q_{S, S}^{R}\right)=v_{\mathfrak{M}}^{R}\left(v_{\mathfrak{M}}^{S} \otimes \vartheta\right)=v_{\mathfrak{M}}^{S}\left(\operatorname{Id}_{\mathfrak{M}} \otimes \Upsilon_{S} \tilde{\vartheta}\right)\left(\operatorname{Id}_{\mathfrak{M}} \otimes q_{S, S}^{R}\right),
$$

and so $\Upsilon_{\mathfrak{M}} \tilde{\vartheta} \bar{\zeta}_{\mathfrak{M}}=v_{\mathfrak{M}}^{S}\left(\operatorname{Id}_{\mathfrak{M}} \otimes \Upsilon_{S} \tilde{\vartheta}\right)$. Hence (3.5) is actually equivalent to

$$
v_{\mathfrak{M}}^{S}\left(\operatorname{Id}_{\mathfrak{M}} \otimes \Upsilon_{S} \tilde{\vartheta} \bar{\zeta}_{S}\left(\operatorname{Id}_{S} \otimes e\right) i\right)=v_{\mathfrak{M}}^{R}, \forall \mathfrak{M} \in \mathcal{C}_{S} .
$$

Notice that if $\Upsilon_{S} \tilde{\vartheta} \bar{\zeta}_{S}\left(\operatorname{Id}_{S} \otimes e\right)=\operatorname{Id}_{S}$ then (3.6) is satisfied since $v_{\mathfrak{M}}^{S}\left(\operatorname{Id}_{\mathfrak{M}} \otimes i\right)=v_{\mathfrak{M}}^{R}$, for all $\mathfrak{M} \in \mathcal{C}_{S}$. The converse is also true since if we get $\mathfrak{M}=S$ in (3.6) with $v_{S}^{S}=\underline{m}_{S}$ and compose it to the right with $\operatorname{Id}_{S} \otimes \underline{\eta}_{R}$ we obtain

$$
\operatorname{Id}_{S}=\underline{m}_{S}\left(\operatorname{Id}_{S} \otimes \Upsilon_{S} \tilde{\vartheta} \bar{\zeta}_{S}\left(\operatorname{Id}_{S} \otimes e\right) \underline{\eta}_{S}\right) .
$$

Straightforward computations lead us to

$$
\underline{m}_{S}\left(\operatorname{Id}_{S} \otimes \Upsilon_{S}\right)\left(\operatorname{Id}_{S} \otimes q_{S, R}^{R}\right)=\underline{m}_{S}\left(\underline{m}_{S} \otimes i\right)=\Upsilon_{S} \mu_{S \otimes_{R} R}^{S}\left(\operatorname{Id}_{S} \otimes q_{S, R}^{R}\right)
$$

and

$$
\mu_{S \otimes_{R} R}^{S}\left(\operatorname{Id}_{S} \otimes \tilde{\vartheta}\right)\left(\operatorname{Id}_{S} \otimes q_{S, S}^{R}\right)=q_{S, R}^{R}\left(\underline{m}_{S} \otimes \vartheta\right)=\tilde{\vartheta} \bar{\zeta}_{S}\left(\operatorname{Id}_{S} \otimes q_{S, S}^{R}\right)
$$


and so we obtain that

$$
\left(\underline{m}_{S}\left(\operatorname{Id}_{S} \otimes \Upsilon_{S}\right)=\Upsilon_{S} \mu_{S \otimes_{R} R}^{S} \text { and } \mu_{S \otimes_{R} R}^{S}\left(\operatorname{Id}_{S} \otimes \tilde{\vartheta}\right)=\tilde{\vartheta} \bar{\zeta}_{S} .\right.
$$

Notice also that

$$
\bar{\zeta}_{S}\left(\operatorname{Id}_{S} \otimes e\right) \underline{\eta}_{S}=\zeta_{S}^{e} \underline{\eta}_{S}=\beta^{-1}\left(\zeta^{e}\right)=\beta^{-1}(\beta(e))=e .
$$

Summing up we get

$$
\operatorname{Id}_{S}=\underline{m}_{S}\left(\operatorname{Id}_{S} \otimes \Upsilon_{S} \tilde{\vartheta} e\right)=\Upsilon_{S} \mu_{S \otimes_{R} R}^{R}\left(\operatorname{Id}_{S} \otimes \tilde{\vartheta} e\right)=\Upsilon_{S} \tilde{\vartheta} \bar{\zeta}_{S}\left(\operatorname{Id}_{S} \otimes e\right),
$$

as needed. Otherwise stated, we have shown that (3.6) is equivalent to

$$
\Upsilon_{S} \tilde{\vartheta} \bar{\zeta}_{S}\left(\operatorname{Id}_{S} \otimes e\right)=\operatorname{Id}_{S}
$$

If we compose this equality to the right with $\underline{\eta}_{S}$ we get

$$
\underline{\eta}_{S}=\Upsilon_{S} \tilde{\vartheta} \bar{\zeta}_{S}\left(\operatorname{Id}_{S} \otimes e\right) \underline{\eta}_{S}=\Upsilon_{S} \tilde{\vartheta} \zeta_{S}^{e} \underline{\eta}_{S}=\Upsilon_{S} \tilde{\vartheta} e
$$

i.e., the second diagram in the statement is commutative. Finally, if $\Upsilon_{S} \tilde{\vartheta} e=\underline{\eta}_{S}$ we then have

$$
\begin{aligned}
\Upsilon_{S} \tilde{\vartheta} \bar{\zeta}_{S}\left(\operatorname{Id}_{S} \otimes e\right) & \stackrel{(3.7)}{=} \Upsilon_{S} \mu_{S \otimes_{R} R}^{S}\left(\operatorname{Id}_{S} \otimes \tilde{\vartheta}\right)\left(\operatorname{Id}_{S} \otimes e\right) \\
& \stackrel{(3.7)}{=} \underline{m}_{S}\left(\operatorname{Id}_{S} \otimes \Upsilon_{S} \tilde{\vartheta} e\right) \\
& =\underline{m}_{S}\left(\operatorname{Id}_{S} \otimes \underline{\eta}_{S}\right)=\operatorname{Id}_{S},
\end{aligned}
$$

and this finishes the proof of the theorem.

The next result generalizes [10, Theorem 27 1.\& 2.] to an algebra extension in a monoidal category.

Theorem 3.6. Let $\mathcal{C}$ be a monoidal category such that 1 is a left $\otimes$-generator and $i: R \rightarrow S$ an algebra morphism in $\mathcal{C}$. Then the following assertions hold:

(i) The restriction of scalars functor $F: \mathcal{C}_{S} \rightarrow \mathcal{C}_{R}$ is separable if and only if there exists a morphism $e \in \mathcal{W}=\left\{e: \underline{1} \rightarrow S \otimes_{R} S \mid \mu_{S \otimes_{R} S}^{S}\left(\operatorname{Id}_{S} \otimes e\right)=\right.$ $\left.\nu_{S \otimes_{R} S}^{S}\left(e \otimes \operatorname{Id}_{S}\right)\right\}$ such that $\underline{m}_{S}^{R} e=\underline{\eta}_{S}$, where $\underline{m}_{S}^{R}: S \otimes_{R} S \rightarrow S$ is the unique morphism in $\mathcal{C}$ obeying $\underline{m}_{S}^{R} q_{S, S}^{R}=\underline{m}_{S}$;

(ii) The induction functor $-\otimes_{R} S: \mathcal{C}_{R} \rightarrow \mathcal{C}_{S}$ is separable if and only if there exists an R-bimodule morphism $\vartheta: S \rightarrow R$ such that $\vartheta \underline{\eta}_{S}=\underline{\eta}_{R}$.

Proof. (i) The functor $-\otimes_{R} S$ is a left adjoint of $F$, so by the Rafael's theorem (see Section 2.3) it follows that $F$ is separable if and only if the counit $\varepsilon$ defined in (3.1) cosplits. By Lemma 3.4 this happens if and only if there is $e \in \mathcal{W}$ such that if

$$
\zeta^{e}=\left(\zeta_{\mathfrak{M}}^{e}=\bar{\zeta}_{\mathfrak{M}}\left(\operatorname{Id}_{\mathfrak{M}} \otimes e\right): \mathfrak{M} \rightarrow \mathfrak{M} \otimes_{R} S\right)_{\mathfrak{M} \in \mathcal{C}_{S}}
$$

is the natural transformation associated to $e$ as in Lemma 3.4 then $\varepsilon \circ \zeta^{e}$ is the identity natural transformation of $1_{\mathcal{C}_{S}}$. Clearly this is equivalent to the existence of 
an element $e \in \mathcal{W}$ such that

$$
\overline{v_{\mathfrak{M}}^{S}} \bar{\zeta}_{\mathfrak{M}}\left(\operatorname{Id}_{\mathfrak{M}} \otimes e\right)=\operatorname{Id}_{\mathfrak{M}}, \quad \forall \mathfrak{M} \in \mathcal{C}_{S}
$$

Since

$\overline{v_{\mathfrak{M}}^{S}} \bar{\zeta}_{\mathfrak{M}}\left(\operatorname{Id}_{\mathfrak{M}} \otimes q_{S, S}^{R}\right)=\overline{v_{\mathfrak{M}}^{S}} q_{\mathfrak{M}, S}^{R}\left(v_{\mathfrak{M}}^{S} \otimes \operatorname{Id}_{S}\right)=v_{\mathfrak{M}}^{S}\left(v_{\mathfrak{M}}^{S} \otimes \underline{m}_{S}\right)=v_{\mathfrak{M}}^{S}\left(\operatorname{Id}_{\mathfrak{M}} \otimes \underline{m}_{S}^{R} q_{S, S}^{R}\right)$

we deduce that $\overline{v_{\mathfrak{M}}^{S}} \bar{\zeta}_{\mathfrak{M}}=v_{\mathfrak{M}}^{S}\left(\operatorname{Id}_{\mathfrak{M}} \otimes \underline{m}_{S}^{R}\right)$. Thus $F$ is separable if and only if there exists $e \in \mathcal{W}$ such that $v_{\mathfrak{M}}^{S}\left(\operatorname{Id}_{\mathfrak{M}} \otimes \underline{m}_{S}^{R} e\right)=\operatorname{Id}_{\mathfrak{M}}$, for all $\mathfrak{M} \in \mathcal{C}_{S}$. If $\underline{m}_{S}^{R} e=\underline{\eta}_{S}$ then the latter condition is clearly satisfied. For the converse take $\mathfrak{M}=S$ and $v_{S}^{S}=\underline{m}_{S}$. We then have $\underline{m}_{S}\left(\operatorname{Id}_{S} \otimes \underline{m}_{S}^{R} e\right)=\operatorname{Id}_{S}$, and therefore

$$
\underline{\eta}_{S}=\underline{m}_{S}\left(\operatorname{Id}_{S} \otimes \underline{m}_{S}^{R} e\right) \underline{\eta}_{S}=\underline{m}_{S}\left(\underline{\eta}_{S} \otimes \operatorname{Id}_{S}\right) \underline{m}_{S}^{R} e=\underline{m}_{S}^{R} e,
$$

as needed.

(ii) Similar to the one given for (i), so we leave it to the reader.

\section{Frobenius and separable algebra extensions in monoidal categories}

Let $k$ be a field and $A$ a $k$-algebra. We say that $A$ is a Frobenius algebra iff $A$ is isomorphic to its $k$-dual $A^{*}:=\operatorname{Hom}_{k}(A, k)$ as a left or right $A$-module, and we call $A$ separable if the multiplication of $A$ cosplits in the category of $A$-bimodules. It is immediate that these definitions can be adapted for an algebra in a monoidal category; note that in the Frobenius case the existence of the dual object of $A$ can be avoided if we use the characterization of a Frobenius algebra given in terms of a Frobenius pair or the one given in the language of Frobenius functors. Concretely, we have the following notions.

Definition 4.1. Let $\mathcal{C}$ be a monoidal category and $A$ an algebra in $\mathcal{C}$.

(i) $A$ is called Frobenius if there exists a pair $(\vartheta, e)$ with $\vartheta: A \rightarrow \underline{1}$ and $e: \underline{1} \rightarrow$ $A \otimes A$ morphisms in $\mathcal{C}$ such that

$\left(\underline{m}_{A} \otimes \operatorname{Id}_{A}\right)\left(\operatorname{Id}_{A} \otimes e\right)=\left(\operatorname{Id}_{A} \otimes \underline{m}_{A}\right)\left(e \otimes \operatorname{Id}_{A}\right),\left(\vartheta \otimes \operatorname{Id}_{A}\right) e=\underline{\eta}_{A}=\left(\operatorname{Id}_{A} \otimes \vartheta\right) e$.

(ii) $A$ is called separable if there exists a morphism $\gamma: A \rightarrow A \otimes A$ of $A$ bimodules such that $\underline{m}_{A} \gamma=\operatorname{Id}_{A}$, where both $A$ and $A \otimes A$ are viewed as $A$ bimodules via the multiplication $\underline{m}_{A}$ of $A$.

Examples 4.2. 1) An algebra in $\underline{\text { Sets }}$ is a monoid. A simple inspection shows that the only monoid carrying out a Frobenius/separable structure is the trivial one.

2) Let $k$ be a commutative ring. An algebra in ${ }_{k} \mathcal{M}$ is an algebra $R$ over $k . R$ is Frobenius/separable in the monoidal sense if and only if it is Frobenius/separable in the classical sense. 
More generally, let $R$ be a $k$-algebra. An algebra in ${ }_{R} \mathcal{M}_{R}$ is a so called $R$-ring. It is well known that there is a one to one correspondence between $R$-rings and $k$ algebra morphisms $i: R \rightarrow S$. Furthermore, the $R$-ring $S$ is Frobenius/separable if and only if the corresponding extension $i: R \rightarrow S$ is so (see Proposition 4.8 below).

3) Particular classes of Frobenius algebras in FdHilb were studied in [12]. Note that any basis of a finite dimensional complex vector space gives rise to a commutative special Frobenius algebra, and that the orthogonal bases of a finite dimensional complex Hilbert space $\mathcal{H}$ are in a one to one correspondence with the so called commutative $\uparrow$-Frobenius algebra structures on $\mathcal{H}$ (for details and terminology we invite the reader to consult [12]).

Also, it has been proved in [16, Theorem 2] that Greenberger-Horne-Zeilinger (GHZ) states are in a one to one correspondence with the special commutative Frobenius algebra structures on the two dimensional Hilbert space $\mathbb{C}^{2}$.

4) Let $k$ be a field and $\mathcal{Z}_{k}$ the category of Zunino over $k$. Algebras in $\mathcal{Z}_{k}$ are in one to one correspondence to algebras graded by a monoid. Since a Frobenius monoid is trivial it follows that a Frobenius algebra in $\mathcal{Z}_{k}$ is a Frobenius $k$-algebra endowed with the trivial grading given by the trivial monoid.

Similarly, a separable algebra in $\mathcal{Z}_{k}$ is a separable $k$-algebra equipped with the trivial grading given by the trivial monoid.

5) Let $k$ be a field and $\mathcal{T}_{k}$ the category of Turaev over $k$. By [11] an algebra $\underline{A}$ in $\mathcal{T}_{k}$ is a family of $k$-algebras $\left(A_{x}\right)_{x \in X}$ indexed by a set $X$. It is easy to see that if such an algebra $\underline{A}$ is Frobenius/separable then $X$ is a singleton. This allows us to show that Frobenius/separable algebras in $\mathcal{T}_{k}$ are in a one to one correspondence with Frobenius/separable algebras over $k$.

6) Let $k$ be a field, $G$ a group and $\phi$ a normalized 3-cocycle on $G$. Then an algebra in $\operatorname{Vect}_{\phi}^{G}$ is precisely a $G$-graded quasialgebra with associator $\phi$ : a $G$-graded vector space $A$ together with a multiplication and a usual unit $1 \in A_{e}$ obeying $(a b) c=\phi(|a|,|b|,|c|) a(b c)$, for all homogeneous elements $a, b, c \in A$, where if $x \in A$ is a homogeneous element then $|x|$ denotes the degree of $x$.

An algebra $A=\bigoplus_{g \in G} A_{g}$ in $\operatorname{Vect}_{\phi}^{G}$ is Frobenius if there exist a $G$-graded morphism $\vartheta: A \rightarrow k$ (so $\vartheta\left(A_{g}\right)=0$, for all $G \ni g \neq e$ ) and an element $e=\sum_{g \in G} a_{g} \otimes b_{g} \in \bigoplus_{g \in G} A_{g} \otimes A_{g^{-1}}$ such that $\vartheta\left(a_{e}\right) b_{e}=\vartheta\left(b_{e}\right) a_{e}=1$ and

$$
\sum_{g \in G} \phi^{-1}\left(|a|, g, g^{-1}\right) a a_{g} \otimes b_{g}=\sum_{g \in G} \phi\left(g, g^{-1},|a|\right) a_{g} \otimes b_{g} a,
$$

for all homogeneous elements $a \in A$. Particular examples of such algebras are Cayley-Dickson and Clifford algebras, cf. $[4,5]$. In fact, they are Frobenius algebras of type $k_{F}[G]$ where $k_{F}[G]$ is the $k$-vector space $k[G]$ endowed with the multiplication $x \bullet y=F(x, y) x y$, for all $x, y \in G$, extended by linearity. Here $F: G \times G \rightarrow k \backslash\{0\}$ is a pointwise invertible map satisfying $F(e, x)=F(x, e)=1$, for all $x \in G$, and $G$ is a finite group having the order $|G|$ non-zero in $k$. 
If $\left\{P_{g}\right\}_{g \in G}$ is the basis in $k_{F}[G]^{*}$ dual to the basis $\{g \mid g \in G\}$ of $k_{F}[G]$ then $(\vartheta, e)$ is given by $\vartheta=|G| P_{e}$ and

$$
e=\frac{1}{|G|} \sum_{g \in G} F\left(g, g^{-1}\right)^{-1} g \otimes g^{-1} .
$$

In particular, we get that if $\sum_{g \in G} F\left(g, g^{-1}\right)^{-1} \neq 0$ in $k$ then a $G$-graded quasialgebra of the form $k_{F}[G]$ is separable in $\operatorname{Vect}_{\Delta_{2}\left(F^{-1}\right)}^{G}$, too. By $\Delta_{2}\left(F^{-1}\right)$ we have denoted the (coboundary) 3-cocycle on $G$ defined, for all $x, y, z \in G$, by

$$
\Delta_{2}\left(F^{-1}\right)(x, y, z)=F(y, z)^{-1} F(x y, z) F(x, y z)^{-1} F(x, y) .
$$

We should also point out that Frobenius algebras in $\mathrm{Vect}^{G}$ (the category $\mathrm{Vect}_{\phi}^{G}$ with $\phi$ equal to the trivial 3-cocycle on $G$ ) have their own theory, recently developed in [14].

7) Let $H$ be a bialgebra over a field $k$. An algebra in ${ }_{H} \mathcal{M}$ is a $k$-algebra $A$ with the multiplication and the unit morphism left $H$-linear morphisms. One can see easily that $A$ is Frobenius in ${ }_{H} \mathcal{M}$ if there exist $\vartheta: A \rightarrow k$ in ${ }_{H} \mathcal{M}$ and $e=e^{1} \otimes e^{2} \in A \otimes A$ such that $\vartheta\left(e^{1}\right) e^{2}=\vartheta\left(e^{2}\right) e^{1}=1$ and

$$
h_{1} \cdot e^{1} \otimes h_{2} \cdot e^{2}=\varepsilon(h) e, \forall h \in H ; a e^{1} \otimes e^{2}=e^{1} \otimes e^{2} a, \forall a \in A .
$$

Likewise, $A$ is separable if there exists an element $e$ as above satisfying (4.2) and $e^{1} e^{2}=1$. Otherwise stated, a Frobenius/separable algebra in ${ }_{H} \mathcal{M}$ is a Frobenius/separable $k$-algebra $A$ for which the Frobenius/separable structure behaves well with respect to the $H$-module structure of $A$.

In a similar manner one can characterize Frobenius/separable algebras in a category of representations over a weak bialgebra, the details are left to the reader.

It is clear that $A$ is Frobenius in $\mathcal{C}$ if and only if the forgetful functor $F: \mathcal{C}_{A} \rightarrow \mathcal{C}$ is Frobenius, provided that $\underline{1}$ is a left $\otimes$-generator for $\mathcal{C}$. Note that in this case we do not have to assume that $\mathcal{C}$ has coequalizers or that any object in $\mathcal{C}$ is coflat, since when the algebra extension is given by the unit morphism $\eta_{A}: \underline{1} \rightarrow A$ the tensor product over the source algebra is just the tensor product $\otimes$ of $\mathcal{C}$.

In the separable case we have a similar result.

Proposition 4.3. Let $\mathcal{C}$ be a monoidal category such that $\underline{1}$ is a left $\otimes$-generator for $\mathcal{C}$. If $A$ is an algebra in $\mathcal{C}$ then $A$ is separable if and only if the forgetful functor $F: \mathcal{C}_{A} \rightarrow \mathcal{C}$ is a separable functor.

Proof. It is straightforward to see that an algebra $A$ is separable if and only if there exists a morphism $e: \underline{1} \rightarrow A \otimes A$ such that

$$
\left(\underline{m}_{A} \otimes \operatorname{Id}_{A}\right)\left(\operatorname{Id}_{A} \otimes e\right)=\left(\operatorname{Id}_{A} \otimes \underline{m}_{A}\right)\left(e \otimes \operatorname{Id}_{A}\right)
$$


and $\underline{m}_{A} e=\underline{\eta}_{A}$. Indeed, for $e$ as above if we define

$$
\gamma=\left(\underline{m}_{A} \otimes \operatorname{Id}_{A}\right)\left(\operatorname{Id}_{A} \otimes e\right)=\left(\operatorname{Id}_{A} \otimes e\right) \underline{m}_{A}
$$

it then follows that $\gamma$ is $A$-bilinear and $\underline{m}_{A} \gamma=\operatorname{Id}_{A}$. Conversely, if $\gamma$ is an $A$ bimodule morphism in $\mathcal{C}$ then $e=\gamma \eta_{A}$ has the desired properties, we leave the details to the reader.

But when 1 is a left $\otimes$-generator in $\mathcal{C}$ the existence of such a morphism $e$ is equivalent to the fact that the functor $F$ is separable, cf. Theorem 3.6. So our proof is complete.

Definition 4.4. If $A$ is a separable algebra in a monoidal category $\mathcal{C}$ we then call the morphism $e: \underline{1} \rightarrow A \otimes A$ satisfying $\left(\underline{m}_{A} \otimes \operatorname{Id}_{A}\right)\left(\operatorname{Id}_{A} \otimes e\right)=\left(\operatorname{Id}_{A} \otimes e\right) \underline{m}_{A}$ and $\underline{m}_{A} e=\underline{\eta}_{A}$ the separability morphism of $A$.

Another characterization for a separable algebra can be given if we assume that the unit object of the category is projective. The result below is a straightforward generalization of the classical result asserting that an algebra $A$ over a commutative ring $k$ is separable if and only if $A$ is a projective $A$-bimodule.

Proposition 4.5. Let $\mathcal{C}$ be a monoidal category having the unit 1 a projective object. Then an algebra $A$ in $\mathcal{C}$ is separable if and only if $A$ is projective as an A-bimodule.

Proof. Assume that $A$ is a separable algebra and let $e: \underline{1} \rightarrow A \otimes A$ be its separability morphism. Consider $\mathfrak{M} \stackrel{p}{\rightarrow} \mathfrak{N} \rightarrow 0$ a short exact sequence of $A$-bimodules in $\mathcal{C}$ and $f: A \rightarrow \mathfrak{N}$ a morphism in ${ }_{A} \mathcal{C}_{A}$. We have to show that there is an $A$-bimodule morphism $g: A \rightarrow \mathfrak{M}$ such that $p g=f$.

If $\tilde{f}:=f \underline{\eta}_{A}$ then clearly $f=\mu_{\mathfrak{N}}^{A}\left(\operatorname{Id}_{A} \otimes \tilde{f}\right)=v_{\mathfrak{N}}^{A}\left(\tilde{f} \otimes \operatorname{Id}_{A}\right)$. Since $\underline{1}$ is projective it follows that there exists a morphism $\tilde{g}: \underline{1} \rightarrow \mathfrak{M}$ in $\mathcal{C}$ such that $p \tilde{g}=\tilde{f}$. So if we define $g: A \rightarrow \mathfrak{M}$ given by

$$
g=v_{\mathfrak{M}}^{A}\left(\mu_{\mathfrak{M}}^{A} \otimes \operatorname{Id}_{A}\right)\left(\operatorname{Id}_{A} \otimes \tilde{g} \otimes \operatorname{Id}_{A}\right)\left(\underline{m}_{A} \otimes \operatorname{Id}_{A}\right)\left(\operatorname{Id}_{A} \otimes e\right)
$$

then $g$ is $A$-bilinear since $\underline{m}_{A}$ is associative, $\left(\underline{m}_{A} \otimes \operatorname{Id}_{A}\right)\left(\operatorname{Id}_{A} \otimes e\right)=\left(\operatorname{Id}_{A} \otimes e\right) \underline{m}_{A}$ and

$$
\begin{aligned}
p g & =v_{\mathfrak{M}}^{A}\left(\mu_{\mathfrak{M}}^{A} \otimes \operatorname{Id}_{A}\right)\left(\operatorname{Id}_{A} \otimes f \underline{\eta}_{A} \otimes \operatorname{Id}_{A}\right)\left(\underline{m}_{A} \otimes \operatorname{Id}_{A}\right)\left(\operatorname{Id}_{A} \otimes e\right) \\
& =v_{\mathfrak{M}}^{A}\left(f \otimes \operatorname{Id}_{A}\right)\left(\underline{m}_{A} \otimes \operatorname{Id}_{A}\right)\left(\operatorname{Id}_{A} \otimes e\right) \\
& =f \underline{m}_{A}\left(\operatorname{Id}_{A} \otimes \underline{m}_{A} e\right)=f \underline{m}_{A}\left(\operatorname{Id}_{A} \otimes \underline{\eta}_{A}\right)=f,
\end{aligned}
$$

as required.

Conversely, if $A$ is projective in ${ }_{A} \mathcal{C}_{A}$ then since $\underline{m}_{A}: A \otimes A \rightarrow A$ is an epimorphism in $\mathcal{C}$ it follows that there exists an $A$-bilinear morphism $\gamma: A \rightarrow A \otimes A$ such that $\underline{m}_{A} \gamma=\operatorname{Id}_{A}$, and so $A$ is a separable algebra. Observe that for this implication we do not need the unit object to be projective. 
Motivated by the above results and the theory of Frobenius (respectively separable) algebra extensions in a category of vector spaces we propose the following terminology. As in the previous section we assume that $\mathcal{C}$ is a monoidal category with coequalizers and such that any object of it is coflat.

Definition 4.6. Let $i: R \rightarrow S$ be an algebra extension in $\mathcal{C}$.

(i) We call the algebra extension $i: R \rightarrow S$ Frobenius if there exist an $R$ bimodule morphism $\vartheta: S \rightarrow R$ and a morphism $e: \underline{1} \rightarrow S \otimes_{R} S$ in $\mathcal{C}$ such that the three conditions stated in Theorem 3.5 (ii) are satisfied. If this is the case then we call the pair $(\vartheta, e)$ the Frobenius pair of the Frobenius extension $i: R \rightarrow S$. Furthermore, we call $\vartheta$ the Frobenius morphism and $e$ the Casimir morphism of the Frobenius algebra extension $i$.

(ii) We call the algebra extension $i: R \rightarrow S$ separable if there exists a morphism $e \in \mathcal{W}=\left\{e: \underline{1} \rightarrow S \otimes_{R} S \mid \mu_{S \otimes_{R} S}^{S}\left(\operatorname{Id}_{S} \otimes e\right)=v_{S \otimes_{R} S}^{S}\left(e \otimes \operatorname{Id}_{S}\right)\right\}$ such that $\underline{m}_{S}^{R} e=\underline{\eta}_{S}$, where $\underline{m}_{S}^{R}: S \otimes_{R} S \rightarrow S$ is the unique morphism in $\mathcal{C}$ obeying $\underline{m}_{S}^{R} q_{S, S}^{R}=\underline{m}_{S}$.

In the case when 1 is a left $\otimes$-generator for $\mathcal{C}$ the definitions above are equivalent to the fact that the functor restriction of scalars is Frobenius, respectively separable. We next show that these notions reduce to the notions of Frobenius, respectively separable, algebra in a suitable monoidal category. To this end we first need a preliminary result.

Lemma 4.7. Let $\mathcal{C}$ be a monoidal category and $i: R \rightarrow S$ an algebra extension in $\mathcal{C}$. Denote by $\mu_{S \otimes_{R} S}^{\prime S}=\widehat{m_{S}^{R}} \Gamma_{S, S, S}^{\prime}$ and $\nu_{S \otimes_{R} S}^{\prime S}=\widetilde{m_{S}^{R}} \Sigma_{S, S, S}^{\prime}$ the canonical morphisms in ${ }_{R} \mathcal{C}_{R}$ that give on $S \otimes_{R} S$ an $S$-bimodule structure in ${ }_{R} \mathcal{C}_{R}$, and by $\underline{m}_{S}^{R}$ the multiplication of the algebra $S$ in ${ }_{R} \mathcal{C}_{R}$. Then there exists a bijection between $\mathcal{W}^{\prime}$, the set of morphisms $\mathfrak{e}: R \rightarrow S \otimes_{R} S$ in ${ }_{R} \mathcal{C}_{R}$ obeying

$$
\mu_{S \otimes_{R} S}^{\prime S} \tilde{\mathfrak{e}} \Upsilon_{S}^{-1}=v_{S \otimes_{R} S}^{\prime S} \hat{\mathfrak{e}} \Upsilon_{S}^{\prime-1}
$$

and

$$
\mathcal{W}=\left\{e: \underline{1} \rightarrow S \otimes_{R} S \mid \mu_{S \otimes_{R} S}^{S}\left(\operatorname{Id}_{S} \otimes e\right)=v_{S \otimes_{R} S}^{S}\left(e \otimes \operatorname{Id}_{S}\right)\right\} .
$$

Proof. By the convention made at the end of Section 2.1 any object of $\mathcal{C}$ is left robust, too.

Let us start by noting that $S$ admits an algebra structure in ${ }_{R} \mathcal{C}_{R}$ if and only if $S$ admits an algebra structure in $\mathcal{C}$ such that the unit morphism $i$ of $S$ in ${ }_{R} \mathcal{C}_{R}$ becomes an algebra morphism in $\mathcal{C}$. Then the $R$-bimodule structure of $S$ coincides with the one induced by $i$.

Also, directly from the definitions it follow that the two structure morphisms $\mu_{S \otimes_{R} S}^{\prime S}$ and $\nu_{S \otimes_{R} S}^{\prime S}$ are completely determined by the equalities

$$
\mu_{S \otimes_{R} S}^{\prime S} q_{S, S \otimes_{R} S}^{R}=\mu_{S \otimes_{R} S}^{S} \quad \text { and } \quad v_{S \otimes_{R} S}^{\prime S} q_{S \otimes_{R} S, S}^{R}=v_{S \otimes_{R} S}^{S},
$$

respectively. 
Now, if $\mathfrak{e}$ is an element of $\mathcal{W}^{\prime}$ we then claim that $e=\mathfrak{e} \underline{\eta}_{R}: \underline{1} \rightarrow S \otimes_{R} S$ belongs to $\mathcal{W}$. Indeed,

$$
\begin{aligned}
\mu_{S \otimes_{R} S}^{\prime S} \tilde{\mathfrak{e}} \Upsilon_{S}^{-1} & =\mu_{S \otimes_{R} S}^{\prime S} \tilde{\mathfrak{e}} q_{R, S}^{R}\left(\operatorname{Id}_{S} \otimes \underline{\eta}_{R}\right) \\
& =\mu_{S \otimes_{R} S}^{\prime S} q_{S, S \otimes_{R} S}^{R}\left(\operatorname{Id}_{S} \otimes \mathfrak{e} \underline{\eta}_{R}\right) \\
& =\mu_{S \otimes_{R} S}^{S}\left(\operatorname{Id}_{S} \otimes e\right),
\end{aligned}
$$

and, similarly, $v_{S \otimes_{R} S}^{\prime S} \hat{\mathfrak{e}} \Upsilon_{S}^{\prime-1}=v_{S \otimes_{R} S}^{S}\left(e \otimes \operatorname{Id}_{S}\right)$, proving the commutativity of the first diagram in the statement (ii) of Theorem 3.5.

Conversely, if $e$ is in $\mathcal{W}$ we then define $\mathfrak{e}=\mu_{S \otimes_{R} S}^{S}(i \otimes e)$. That $\mathfrak{e}$ is left $R$-linear follows easily from the fact that $\mu_{S \otimes_{R} S}^{S}$ defines a left $S$-module structure on $S \otimes_{R} S$ and since $i$ is an algebra map, the details are left to the reader. It is also right $R$-linear since

$$
\begin{aligned}
v_{S \otimes_{R} S}^{R}\left(\mathfrak{e} \otimes \operatorname{Id}_{R}\right) & =v_{S \otimes_{R} S}^{S}\left(\mu_{S \otimes_{R} S}^{S}\left(\operatorname{Id}_{S} \otimes e\right) i \otimes i\right) \\
& =v_{S \otimes_{R} S}^{S}\left(v_{S \otimes_{R} S}^{S}\left(e \otimes \operatorname{Id}_{S}\right) i \otimes i\right) \\
& =v_{S \otimes_{R} S}^{S}\left(e \otimes \operatorname{Id}_{S}\right) \underline{m}_{S}(i \otimes i) \\
& =\mu_{S \otimes_{R} S}^{S}\left(\operatorname{Id}_{S} \otimes e\right) i \underline{m}_{R}=\mathfrak{e} \underline{m}_{R},
\end{aligned}
$$

as needed. Furthermore, since

$$
\left.\mathfrak{e} \underline{\eta}_{R}=\mu_{S \otimes_{R} S}^{S}(i \otimes e) \underline{\eta}_{R}=\mu_{S \otimes_{R} S}^{S} \underline{\eta}_{S} \otimes \operatorname{Id}_{S \otimes_{R} S}\right) e=e,
$$

as in the case of the first correspondence one have that

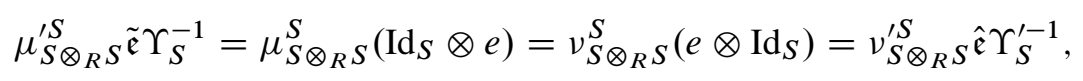

and so $\mathfrak{e}$ is an element in $\mathcal{W}^{\prime}$. We also have shown that $\mathcal{W} \ni e \mapsto \mathfrak{e} \mapsto \mathfrak{e} \underline{\eta}_{R}=e$. Using that an element $\mathfrak{e}$ is left $R$-linear we get that

$$
\mathcal{W}^{\prime} \ni \mathfrak{e} \mapsto e=\mathfrak{e} \underline{\eta}_{R} \mapsto \mu_{S \otimes_{R} S}^{S}(i \otimes \mathfrak{e})\left(\operatorname{Id}_{R} \otimes \underline{\eta}_{R}\right)=\mathfrak{e} \underline{m}_{R}\left(\operatorname{Id}_{R} \otimes \underline{\eta}_{R}\right)=\mathfrak{e},
$$

and this finishes our proof.

Proposition 4.8. Let $\mathcal{C}$ be a monoidal category and $i: R \rightarrow S$ an algebra extension in $\mathcal{C}$. Then the following assertions hold.

(i) The extension $i: R \rightarrow S$ is Frobenius if and only if $S$ is a Frobenius algebra in ${ }_{R} \mathcal{C}_{R}$.

(ii) The extension $i: R \rightarrow S$ is separable if and only if $S$ is a separable algebra in ${ }_{R} \mathcal{C}_{R}$. 
Proof. (i) By Definition 4.1 applied to $S \in{ }_{R} \mathcal{C}_{R}$ we have that $S$ is a Frobenius algebra in ${ }_{R} \mathcal{C}_{R}$ if and only if there exists a pair $(\vartheta, \mathfrak{e})$ with $\vartheta: S \rightarrow R$ and $\mathfrak{e}: R \rightarrow S \otimes_{R} S$ morphisms in ${ }_{R} \mathcal{C}_{R}$ such that

$$
\mu_{S \otimes_{R} S}^{\prime S} \tilde{\mathfrak{e}} \Upsilon_{S}^{-1}=v_{S \otimes_{R}}^{\prime S} S^{\hat{\mathfrak{e}}} \Upsilon_{S}^{\prime-1}, \Upsilon_{S} \tilde{\vartheta} \mathfrak{e}=i \text {, and } \Upsilon_{S}^{\prime} \hat{\vartheta} \mathfrak{e}=i,
$$

where $\mu_{S \otimes_{R} S}^{\prime S}$ and $v_{S \otimes_{R} S}^{\prime S}$ are the morphisms in ${ }_{R} \mathcal{C}_{R}$ defined in the statement of Lemma 4.7.

We show now that to give a pair $(\vartheta, \mathfrak{e})$ as above is equivalent to give a pair $(\vartheta, e)$ with $\vartheta: S \rightarrow R$ a morphism in ${ }_{R} \mathcal{C}_{R}$ and $e: \underline{1} \rightarrow S \otimes_{R} S$ a morphism in $\mathcal{C}$ such that the three diagrams in Theorem 3.5 (ii) are commutative, and this will end the proof.

Indeed, $e$ can be obtained from $\mathfrak{e}$ as $e=\mathfrak{e} \underline{\eta}_{R}$. Then by Lemma 4.7 the first diagram in Theorem 3.5 (ii) is commutative; the commutativity of the other two follow easily by composing the remaining properties of $\mathfrak{e}$ to the right with $\underline{\eta}_{R}$.

Conversely, to a pair $(\vartheta, e)$ corresponds $(\vartheta, \mathfrak{e})$ with $\mathfrak{e}=\mu_{S \otimes_{R} S}^{S}(i \otimes e)$. The first required property on $\mathfrak{e}$ is satisfied because of Lemma 4.7. Moreover, by a direct computation we can check that

$$
\begin{aligned}
\underline{m}_{S}\left(i \otimes \operatorname{Id}_{S}\right)\left(\operatorname{Id}_{R} \otimes \Upsilon_{S} \tilde{\vartheta}\right)\left(\operatorname{Id}_{R} \otimes q_{S, S}^{R}\right) & =\underline{m}_{S}\left(\underline{m}_{S}\left(i \otimes \operatorname{Id}_{S}\right) \otimes i \vartheta\right) \\
& =\Upsilon_{S} \tilde{\vartheta} \mu_{S \otimes_{R} S}^{R}\left(\operatorname{Id}_{R} \otimes q_{S, S}^{R}\right),
\end{aligned}
$$

and thus $\underline{m}_{S}\left(i \otimes \operatorname{Id}_{S}\right)\left(\operatorname{Id}_{R} \otimes \Upsilon_{S} \tilde{\vartheta}\right)=\Upsilon_{S} \tilde{\vartheta} \mu_{S \otimes_{R} S}^{R}$, since $R$ is right coflat, too. We then have

$$
\begin{aligned}
\Upsilon_{S} \tilde{\vartheta}_{\mathfrak{e}} & =\Upsilon_{S} \tilde{\vartheta} \mu_{S \otimes_{R} S}^{R}\left(\operatorname{Id}_{R} \otimes e\right) \\
& =\underline{m}_{S}\left(i \otimes \operatorname{Id}_{S}\right)\left(\operatorname{Id}_{R} \otimes \Upsilon_{S} \tilde{\vartheta} e\right) \\
& =\underline{m}_{S}\left(\operatorname{Id}_{S} \otimes \underline{\eta}_{S}\right) i=i,
\end{aligned}
$$

as required. In a similar manner we can show that $\Upsilon_{S}^{\prime} \hat{\vartheta}_{\mathfrak{e}}=i$, we leave the verification of the details to the reader.

(ii) Similar to the proof of (i). By Definition $4.1 S$ is a separable algebra in ${ }_{R} \mathcal{C}_{R}$ if and only if there exists $\mathfrak{e} \in \mathcal{W}^{\prime}$ such that $\underline{m}_{S}^{R} \mathfrak{e}=i$. Hence it is sufficient to show that the bijection in Lemma 4.7 behaves well with respect to the extra property of $\mathfrak{e}$. So we show that giving an element $\mathfrak{e} \in \mathcal{W}^{\prime}$ such that $\underline{m}_{S}^{R} \mathfrak{e}=i$ is equivalent to giving an element $e \in \mathcal{W}$ such that $\underline{m}_{S}^{R} e=\underline{\eta}_{S}$.

Indeed, on one hand, to $\mathfrak{e} \in \mathcal{W}^{\prime}$ corresponds $e=\mathfrak{e} \underline{\eta}_{R}$, and so $\underline{m}_{S}^{R} e=\underline{m}_{S}^{R} \mathfrak{e} \underline{\eta}_{R}=$ $i \underline{\eta}_{R}=\underline{\eta}_{S}$, as desired. On the other hand, if $e \in \mathcal{W}$ such that $\underline{m}_{S}^{R} e=\underline{\eta}_{S}$ then since $\mathfrak{e}=\mu_{S \otimes_{R} S}^{S}(i \otimes e)$ we have

$$
\underline{m}_{S}^{R} \mathfrak{e}=\underline{m}_{S}^{R} \mu_{S \otimes_{R} S}^{S}(i \otimes e) \stackrel{(*)}{=} \underline{m}_{S}\left(\operatorname{Id}_{S} \otimes \underline{m}_{S}^{R} e\right) i=\underline{m}_{S}\left(\operatorname{Id}_{S} \otimes \underline{\eta}_{S}\right) i=i,
$$

as needed. Note that $(*)$ is a consequence of the fact that $\underline{m}_{S}^{R} \mu_{S \otimes_{R} S}^{S}\left(\operatorname{Id}_{S} \otimes q_{S, S}^{R}\right)=$ $\underline{m}_{S}\left(\underline{m}_{S} \otimes \operatorname{Id}_{S}\right)=\underline{m}_{S}\left(\operatorname{Id}_{S} \otimes \underline{m}_{S}^{R} q_{S, S}^{R}\right)$. 
Corollary 4.9. Let $\mathcal{C}$ be a monoidal category such that 1 is either a projective object or a left $\otimes$-generator for $\mathcal{C}$. If $i: R \rightarrow S$ is an algebra extension in $\mathcal{C}$ with $R$ separable then $S$ is separable if and only if the algebra extension $i: R \rightarrow S$ is separable.

Proof. Assume first that 1 is projective. By Proposition 4.5 we know that $R$, the unit object of ${ }_{R} \mathcal{C}_{R}$, is projective in ${ }_{R} \mathcal{C}_{R}$. Hence the algebra extension $i: R \rightarrow S$ is separable if and only if $S$ is a separable algebra in ${ }_{R} \mathcal{C}_{R}$, if and only if $S$ is projective as an $S$-bimodule in ${ }_{R} \mathcal{C}_{R}$. But ${ }_{S}\left({ }_{R} \mathcal{C}_{R}\right)_{S}$ identifies with ${ }_{S} \mathcal{C}_{S}$, as any object of ${ }_{S}\left({ }_{R} \mathcal{C}_{R}\right)_{S}$ inherits the $R$-module structures from the $S$-module ones (due to the $R$ balanced conditions). Thus the algebra extension $i: R \rightarrow S$ is separable if and only if $S$ is projective in ${ }_{S} \mathcal{C}_{S}$, and since 1 is projective this is equivalent to the fact that $S$ is a separable algebra.

Assume now that 1 is a left $\otimes$-generator for $\mathcal{C}$. Since $R$ is a separable algebra in $\mathcal{C}$ it follows by Proposition 4.3 that the forgetful functor $F^{\prime}: \mathcal{C}_{R} \rightarrow \mathcal{C}$ is separable. Now, the extension $i: R \rightarrow S$ is separable if and only if the restriction of scalars functor $F: \mathcal{C}_{S} \rightarrow \mathcal{C}_{R}$ is separable, cf. Theorem 3.6, and again by Proposition 4.3 we have that $S$ is a separable algebra in $\mathcal{C}$ if and only if the forgetful functor $F^{\prime \prime}: \mathcal{C}_{S} \rightarrow \mathcal{C}$ is separable.

Thus, if the extension $i: R \rightarrow S$ is separable then according to [10, Proposition 46 (1)] the functor $F^{\prime \prime}=F^{\prime} \circ F$ is separable, and so $S$ is a separable algebra in $\mathcal{C}$. Conversely, if $S$ is separable then $F^{\prime \prime}$ is a separable functor and since $F^{\prime \prime}=F^{\prime} \circ F$ by [10, Proposition $\left.46(2)\right]$ we get that $F$ is a separable functor, and hence the extension $i: R \rightarrow S$ is separable.

A result of Eilenberg and Nakayama asserts that any separable algebra over a field is a Frobenius algebra (even more, it is a symmetric algebra, see [17]). As we explained in Introduction, it is an open (and quite difficult) problem to see if this result remains true in the setting of monoidal categories. However, what one can prove is a sort of converse for the above result. It measures how far is a Frobenius algebra from being separable.

Proposition 4.10. Let $A$ be a Frobenius algebra in a monoidal category $\mathcal{C}$ and $(v, e)$ a Frobenius pair for $A$. Then $A$ is separable if and only if there exists a morphism $\alpha: \underline{1} \rightarrow A$ such that $\underline{m}_{A}\left(\underline{m}_{A} \otimes \operatorname{Id}_{A}\right)\left(\operatorname{Id}_{A} \otimes \alpha \otimes \operatorname{Id}_{A}\right) e=\underline{\eta}_{A}$.

Proof. Assume that $A$ is separable and let $\mathbf{e}: \underline{1} \rightarrow A \otimes A$ be a separability morphism for it. If we define $\alpha:=\left(\operatorname{Id}_{A} \otimes v\right) \mathbf{e}: \underline{1} \rightarrow A$ then

$$
\begin{aligned}
& \left(\underline{m}_{A} \otimes \operatorname{Id}_{A}\right)\left(\operatorname{Id}_{A} \otimes \alpha \otimes \operatorname{Id}_{A}\right) e=\left(\underline{m}_{A} \otimes \operatorname{Id}_{A}\right)\left(\operatorname{Id}_{A \otimes A} \otimes v \otimes \operatorname{Id}_{A}\right)\left(\operatorname{Id}_{A} \otimes \mathbf{e} \otimes \operatorname{Id}_{A}\right) e \\
& =\left(\mathrm{Id}_{A} \otimes v \otimes \mathrm{Id}_{A}\right)\left(\left(\underline{m}_{A} \otimes \mathrm{Id}_{A}\right)\left(\mathrm{Id}_{A} \otimes \mathbf{e}\right) \otimes \mathrm{Id}_{A}\right) e \\
& =\left(\operatorname{Id}_{A} \otimes v \otimes \operatorname{Id}_{A}\right)\left(\operatorname{Id}_{A} \otimes \underline{m}_{A} \otimes \operatorname{Id}_{A}\right)\left(\operatorname{Id}_{A \otimes A} \otimes e\right) \mathbf{e} \\
& =\left(\operatorname{Id}_{A} \otimes\left(v \otimes \operatorname{Id}_{A}\right)\left(\operatorname{Id}_{A} \otimes \underline{m}_{A}\right)\left(e \otimes \operatorname{Id}_{A}\right)\right) \mathbf{e}
\end{aligned}
$$




$$
\begin{aligned}
& =\left(\operatorname{Id}_{A} \otimes \underline{m}_{A}\left(\left(v \otimes \operatorname{Id}_{A}\right) e \otimes \operatorname{Id}_{A}\right)\right) \mathbf{e} \\
& =\left(\operatorname{Id}_{A} \otimes \underline{m}_{A}\left(\underline{\eta}_{A} \otimes \operatorname{Id}_{A}\right)\right) \mathbf{e}=\mathbf{e} .
\end{aligned}
$$

From here we get that $\underline{m}_{A}\left(\underline{m}_{A} \otimes \operatorname{Id}_{A}\right)\left(\operatorname{Id}_{A} \otimes \alpha \otimes \operatorname{Id}_{A}\right) e=\underline{m}_{A} \mathbf{e}=\underline{\eta}_{A}$, as desired.

Conversely, let $\alpha: \underline{1} \rightarrow A$ be a morphism in $\mathcal{C}$ satisfying the condition in the statement. Then a simple computation ensures us that

$$
\mathbf{e}:=\left(\underline{m}_{A} \otimes \operatorname{Id}_{A}\right)\left(\operatorname{Id}_{A} \otimes \alpha \otimes \operatorname{Id}_{A}\right) e: \underline{1} \rightarrow A \otimes A
$$

is a separability morphism for $A$, and so $A$ is separable.

Corollary 4.11. Let $i: R \rightarrow S$ be a Frobenius algebra extension in a monoidal category $\mathcal{C}$. Then the extension $i: R \rightarrow S$ is separable if and only if there exists a morphism $\alpha_{0}: \underline{1} \rightarrow S$ in $\mathcal{C}$ such that

$$
\underline{m}_{S}\left(i \otimes \alpha_{0}\right)=\underline{m}_{S}\left(\alpha_{0} \otimes i\right) \text { and } \underline{m}_{S}^{R} \widehat{\widehat{m}}_{S} \widehat{\operatorname{Id}_{S} \otimes \alpha_{0}} e=\underline{\eta}_{S},
$$

where e $: \underline{1} \rightarrow S \otimes_{R} S$ is the morphism in $\mathcal{C}$ associated to the Frobenius extension $i: R \rightarrow S$ as in the proof of Proposition 4.8.

Proof. By Proposition 4.8 we know that $i: R \rightarrow S$ is a Fobenius/separable extension if and only if $S$ is a Frobenius/separable algebra in ${ }_{R} \mathcal{C}_{R}$. If we denote by $(v, \mathbf{e})$ the Frobenius system of $S$ in ${ }_{R} \mathcal{C}_{R}$ then $S$ is separable in ${ }_{R} \mathcal{C}_{R}$ if and only if there exists $\alpha: R \rightarrow S$ an $R$-bimodule morphism in $\mathcal{C}$ such that

$$
\underline{m}_{S}^{R} \underline{\underline{m}}_{S}^{R \widetilde{\alpha} \Upsilon_{S}^{-1}} \mathbf{e}=i
$$

Clearly $\alpha$ is uniquely determined by a morphism $\alpha_{0}: \underline{1} \rightarrow S$ obeying $\underline{m}_{S}\left(i \otimes \alpha_{0}\right)=$ $\underline{m}_{S}\left(\alpha_{0} \otimes i\right)$. Also, by Lemma 4.7 we have that $\mathbf{e}$ is completely determined by $e=\mathbf{e} \underline{\eta}_{R}: \underline{1} \rightarrow S \otimes_{R} S$, an element in $\mathcal{W}$. Finally, since $\underline{m}_{S}^{R} \tilde{\alpha} \Upsilon_{S}^{-1}=\underline{m}_{S}\left(\operatorname{Id}_{S} \otimes \alpha_{0}\right)$ it follows that $S$ is a separable algebra in ${ }_{R} \mathcal{C}_{R}$ if and only if there exists $\alpha_{0}: 1 \rightarrow S$ a morphism in $\mathcal{C}$ satisfying the two conditions in the statement. So we are done.

\section{Further characterizations for monoidal Frobenius algebra extensions}

In view of Proposition 4.8 it is clear that characterizing Frobenius or separable algebra extensions is equivalent to characterizing Frobenius or separable algebras, of course if some coflatness and robustness properties are satisfied. We will do this in the next results. In the Frobenius case we do not have to assume from the beginning that the category is rigid (see the definition below). As we will see the existence of the dual object for a Frobenius algebra in a monoidal catgory $\mathcal{C}$ is automatic. The result can be viewed as the analogue of the classical result asserting that a Frobenius algebra in a category of vector spaces is always finite dimensional. 
Recall that an object $X$ of a monoidal category $\mathcal{C}$ admits a left dual ${ }^{1}$ if there exist an object $X^{*}$ in $\mathcal{C}$ and morphisms ev $X: X^{*} \otimes X \rightarrow \underline{1}$ and $\operatorname{coev}_{X}: \underline{1} \rightarrow X \otimes X^{*}$ in $\mathcal{C}$ such that

$$
\left(\operatorname{Id}_{X} \otimes \mathrm{ev}_{X}\right)\left(\operatorname{coev}_{X} \otimes \operatorname{Id}_{X}\right)=\operatorname{Id}_{X} \text { and }\left(\mathrm{ev}_{X} \otimes \operatorname{Id}_{X^{*}}\right)\left(\operatorname{Id}_{X^{*}} \otimes \operatorname{coev}_{X}\right)=\operatorname{Id}_{X^{*}}
$$

In what follows we denote $\operatorname{ev}_{X}=\frac{X^{*} X}{\underline{\bigcup}}$ and $\operatorname{coev}_{X}=\frac{\frac{1}{\bigcap}}{X X^{*}}$. Hence, the following relations hold:

$$
\frac{X}{\bigcap^{X}}=\frac{X}{X} \text { and } \frac{X^{*}}{\bigcup_{X^{*}}}=\frac{X^{*}}{X^{*}}
$$

If any object of $\mathcal{C}$ admits a left dual we then say that $\mathcal{C}$ is left rigid.

Likewise, $\mathcal{C}$ is right rigid if for any $X \in \mathcal{C}$ there exist an object ${ }^{*} X \in \mathcal{C}$ and morphisms $\mathrm{ev}_{X}^{\prime}: X \otimes{ }^{*} X \rightarrow \underline{1}$ and $\operatorname{coev}_{X}^{\prime}: \underline{1} \rightarrow^{*} X \otimes X$ such that

$$
\left(\mathrm{ev}_{X}^{\prime} \otimes \mathrm{Id}_{X}\right)\left(\mathrm{Id}_{X} \otimes \operatorname{coev}_{X}^{\prime}\right)=\operatorname{Id}_{X} \text { and }\left(\mathrm{Id} *_{X} \otimes \mathrm{ev}_{X}^{\prime}\right)\left(\operatorname{coev}_{X}^{\prime} \otimes \operatorname{Id} *_{X}\right)=\operatorname{Id} *_{X}
$$

In what follows we will denote $\operatorname{ev}_{X}^{\prime}:=\frac{X^{*} X}{\underline{\varphi}}$ and $\operatorname{coev}_{X}^{\prime}:=\frac{\frac{1}{\varrho}}{{ }^{*} X X}$. Then the relations above can be written as

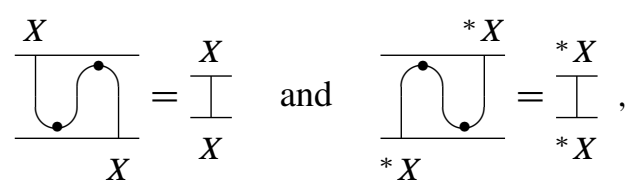

respectively. Thus a right dual for $X$ in $\mathcal{C}$ is nothing but a left dual for $X$ in $\overline{\mathcal{C}}$, the reverse monoidal category associated to $\mathcal{C}(\overline{\mathcal{C}}$ is the category $\mathcal{C}$ endowed with the reverse monoidal structure of $\mathcal{C}$, that is, with the tensor product $\bar{\otimes}=\otimes \circ \tau$, where $\tau: \mathcal{C} \times \mathcal{C} \rightarrow \mathcal{C} \times \mathcal{C}$ is the switch functor). In what follows by $(\rho, \lambda): Y \dashv X$ we denote the fact that $Y$ with $\rho: \underline{1} \rightarrow X \otimes Y$ and $\lambda: Y \otimes X \rightarrow \underline{1}$ is a left dual for $X$ or, equivalently, that $(X, \rho, \lambda)$ is a right dual for $Y$. The pair $(\rho, \lambda)$ is called an adjunction between $Y$ and $X$.

\footnotetext{
${ }^{1}$ In the literature, what we refer to as a left duality is called a right duality, and vice-versa. We adopt here the terminology from [25].
} 
Consider $A$ an algebra in a monoidal category $\mathcal{C}$ that has a left dual object $A^{*}$ (respectively a right dual object ${ }^{*} A$ ). Then it is well known that $A^{*}$ (respectively ${ }^{*} A$ ) is a right (left) $A$-module via the structure morphism
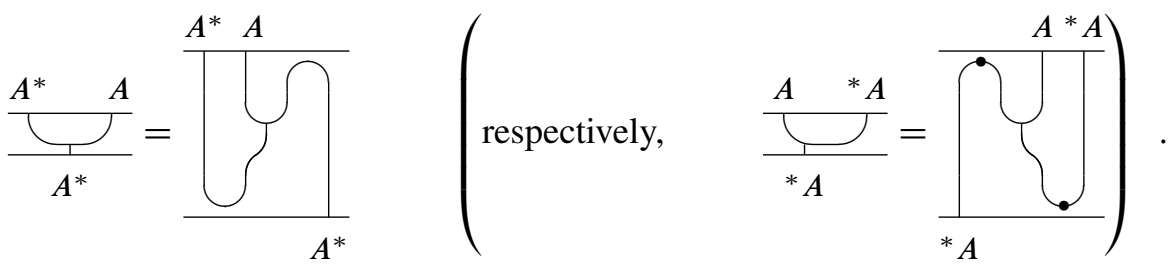

Finally, if $X$ is an arbitrary object of $\mathcal{C}$ and $B: A \otimes A \rightarrow X$ is a morphism in $\mathcal{C}$ we then say that $B$ is associative if and only if $B\left(\underline{m}_{A} \otimes \operatorname{Id}_{A}\right)=B\left(\operatorname{Id}_{A} \otimes \underline{m}_{A}\right)$.

One can now present characterizations for Frobenius algebras in arbitrary monoidal categories, so not necessarily rigid monoidal. Most of them are collected from $[20,48,49,55]$.

Theorem 5.1. Let $\mathcal{C}$ be a monoidal category and $A$ an algebra in $\mathcal{C}$. Then the following assertions are equivalent:

(i) $A$ is a Frobenius algebra;

(ii) A admits a left dual $A^{*}$ and $A$ is isomorphic to $A^{*}$ as a right $A$-module;

(iii) A admits a right dual ${ }^{*} A$ and $A$ is isomorphic to ${ }^{*} A$ as a left A-module;

(iv) A admits a coalgebra structure $\left(A, \underline{\Delta}_{A}, \underline{\varepsilon}_{A}\right)$ in $\mathcal{C}$ such that $\underline{\Delta}_{A}$ is an A-bimodule map, where both $A$ and $A \otimes A$ are considered bimodules via the multiplication $\underline{m}_{A}$ of $A$;

(v) A admits a right dual ${ }^{*} A$ and there exists a morphism $B: A \otimes A \rightarrow \underline{1}$ that is associative and such that $\Phi_{B}^{r}:=\left(\mathrm{Id}_{A} \otimes B\right)\left(\operatorname{coev}_{A}^{\prime} \otimes \mathrm{Id}_{A}\right): A \rightarrow{ }^{*} A$ is an isomorphism in $\mathcal{C}$;

(vi) A admits a left dual $A^{*}$ and there exists a morphism $B: A \otimes A \rightarrow \underline{1}$ in $\mathcal{C}$ that is associative and such that $\Phi_{B}^{l}:=\left(B \otimes \operatorname{Id}_{A^{*}}\right)\left(\operatorname{Id}_{A} \otimes \operatorname{coev}_{A}\right): A \rightarrow A^{*}$ is an isomorphism;

(vii) There exists an adjunction $(\rho, \lambda): A \dashv A$ for which $\lambda: A \otimes A \rightarrow \underline{1}$ is associative;

(viii) There exists an adjunction $(\rho, \lambda): A \dashv A$ such that $\lambda=\vartheta \underline{m}_{A}$ for some $\vartheta: A \rightarrow \underline{1}$ morphism in $\mathcal{C}$.

Furthermore, if $\underline{1}$ is a left $\otimes$-generator then the above assertions are also equivalent to

(ix) $-\otimes A: \mathcal{C} \rightarrow \mathcal{C}_{A}$ is a right adjoint for the forgetful functor $F: \mathcal{C}_{A} \rightarrow \mathcal{C}$ or, in other words, $F$ is a Frobenius functor. 
Proof. We sketch the proof. For each implication below the complete proof can be found in the quoted references or can be done directly by the reader.

(i) $\Leftrightarrow(i i)$. This is pointed out in [55, Proposition 2.1]. Consider $(\vartheta, e)$ a Frobenius system for $A$ and $\operatorname{define~}^{\mathrm{ev}}{ }_{A}=\vartheta \underline{\mathrm{m}}_{A}: A \otimes A \rightarrow \underline{1}$ and $\operatorname{coev}_{A}=e$ : $1 \rightarrow A \otimes A$. Then one can see easily that $\left(A, \mathrm{ev}_{A}, \operatorname{coev}_{A}\right)$ is a left dual for $A$ and, moreover, that the right action of $A$ on this left dual coincides with the multiplication of $A$. Then $A$ has a left dual and is isomorphic to it as a right $A$-module.

For the converse, let $\Psi: A \rightarrow A^{*}$ be a right $A$-linear isomorphism in $\mathcal{C}$ and define

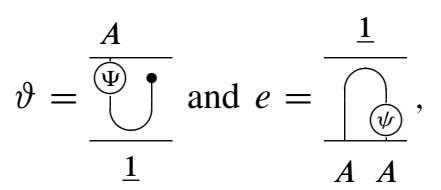

where $\psi$ is the inverse of $\Psi$. Then $(\vartheta, e)$ is a Frobenius pair for $A$, and therefore $A$ is a Frobenius algebra.

(ii ) $\Leftrightarrow($ i i i $)$. Follows from [20, Lemma 5].

(ii) $\Leftrightarrow(i v)$. See for instance [20, Propositions 8 \& 9]. A direct proof, based on a monoidal approach, is the following.

Let $A^{*}$ be a left dual object for $A$ and $\Psi: A \rightarrow A^{*}$ a right $A$-module isomorphism in $\mathcal{C}$. Since the left dual functor $)^{*}: \mathcal{C} \rightarrow \overline{\mathcal{C}}^{\text {opp }}$ is monoidal the left dual $A^{*}$ admits a coalgebra structure in $\overline{\mathcal{C}}$, and therefore in $\mathcal{C}$ as well. If we transport this coalgebra structure on $A^{*}$ through the isomorphism $\Psi$ we get that $A$ admits a coalgebra structure in $\mathcal{C}$. More precisely, with

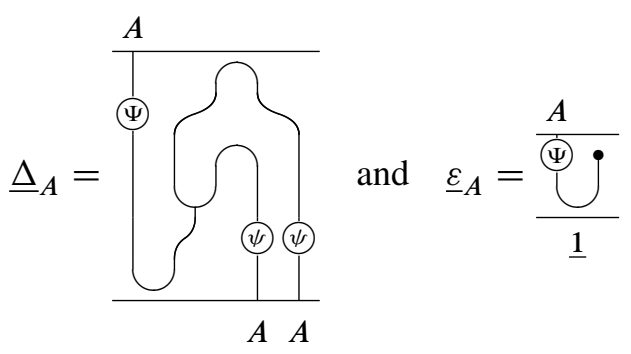

$A$ becomes a coalgebra in $\mathcal{C}$ where, as before, $\psi$ stands for the inverse of $\Psi$. Using that $\Psi$ is right $A$-linear we get that $\underline{\Delta}_{A}$ is an $A$-bimodule morphism in $\mathcal{C}$, as desired.

Conversely, if $A$ admits a coalgebra structure $\left(A, \underline{\Delta}_{A}, \underline{\varepsilon}_{A}\right)$ such that $\underline{\Delta}_{A}$ is an $A$-bilinear morphism then $A$ itself together with $\mathrm{ev}_{A}=\underline{\varepsilon}_{A} \underline{m}_{A}: A \otimes A \rightarrow \underline{1}$ and $\operatorname{coev}_{A}=\underline{\Delta}_{A} \underline{\eta}_{A}: \underline{1} \rightarrow A \otimes A$ is a left dual for $A$. Furthermore, the right action of $A$ on this left dual of it is just $\underline{m}_{A}$. Thus $A$ admits a left dual and is isomorphic to it as a right $A$-module.

(iii) $\Leftrightarrow(v)$ and $($ ii $) \Leftrightarrow(v i)$ follow from [20, Proposition 9].

The implication $(v i i) \Rightarrow(v i)$ is trivial. To prove $(v i) \Rightarrow(v i i)$ we proceed as in the proof of [48, Theorem 1.6] or [49, Theorem]. Namely, if $A^{*}$ is a left dual object 
for $A$ and $\Psi: A \rightarrow A^{*}$ is a right $A$-module isomorphism with inverse $\psi$ then it can be easily verified that $\left(\left(\operatorname{Id}_{A} \otimes \psi\right) \operatorname{coev}_{A}, \mathrm{ev}_{A}\left(\Psi \otimes \operatorname{Id}_{A}\right)\right): A \dashv A$ is an adjunction for which $\mathrm{ev}_{A}\left(\Psi \otimes \operatorname{Id}_{A}\right): A \otimes A \rightarrow \underline{1}$ is associative.

(vii) $\Rightarrow(v i i i)$. If $\lambda$ is associative then $\vartheta:=\lambda\left(\underline{\eta}_{A} \otimes \operatorname{Id}_{A}\right)=\lambda\left(\operatorname{Id}_{A} \otimes \underline{\eta}_{A}\right)$ is the desired morphism since $\vartheta \underline{m}_{A}=\lambda$. The converse is also true because $\vartheta \underline{m}_{A}$ is clearly associative.

The equivalence between (i) and (ix) follows from the comments made before Proposition 4.3, so our proof is finished.

Since Frobenius algebra extensions are particular cases of Frobenius algebras in monoidal categories we get the following list of characterizations for a Frobenius algebra extension.

Corollary 5.2. Let $i: R \rightarrow S$ be an algebra extension in $\mathcal{C}$. Then the following assertions are equivalent:

(i) The extension $i: R \rightarrow S$ is Frobenius;

(ii) $S$ admits a left dual object $S \sqrt{ }$ in ${ }_{R} \mathcal{C}_{R}$ and $S$ and $S \sqrt{ }$ are isomorphic as right $S$-modules in ${ }_{R} \mathcal{C}_{R}$;

(iii) $S$ admits a right dual object $\sqrt{ } S$ in ${ }_{R} \mathcal{C}_{R}$ and $S$ and $\sqrt{ } S$ are isomorphic as left $S$-modules in ${ }_{R} \mathcal{C}_{R}$;

(iv) $S$ admits a coalgebra structure in ${ }_{R} \mathcal{C}_{R}$, that is an $R$-coring structure, such that the comultiplication morphism is $S$-bilinear in ${ }_{R} \mathcal{C}_{R}$.

(v) $S$ admits a right dual $\sqrt{ } S$ in ${ }_{R} \mathcal{C}_{R}$ and there exists a morphism $B: S \otimes_{R} S \rightarrow R$ in ${ }_{R} \mathcal{C}_{R}$ that is associative and such $\Phi_{B}^{r}:=\Upsilon_{\sqrt{ } S} \widetilde{B} \Sigma_{\sqrt{ } S, S, S}^{\prime} \widehat{\operatorname{coev}_{\mathrm{S}}^{\prime}} \Upsilon_{S}^{\prime}-1: S \rightarrow$ $\sqrt{ } S$ is an isomorphism in ${ }_{R} \mathcal{C}_{R}$;

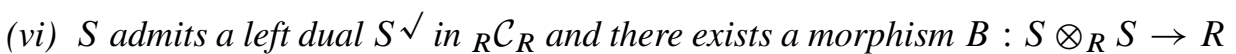
in ${ }_{R} \mathcal{C}_{R}$ that is associative and such that $\Phi^{l}:=\Upsilon_{S}^{\prime} \sqrt{B} \Gamma_{S, S, S}^{\prime} \sqrt{\operatorname{coev}_{S}} \Upsilon_{S}^{-1}$ : $S \rightarrow S^{\sqrt{ }}$ is an isomorphism in ${ }_{R} \mathcal{C}_{R}$;

(vii) There exists an adjunction $(\rho, \lambda): S \dashv S$ in ${ }_{R} \mathcal{C}_{R}$ for which $\lambda: S \otimes_{R} S \rightarrow R$ is associative;

(viii) There exists an adjunction $(\rho, \lambda): S \dashv S$ in ${ }_{R} \mathcal{C}_{R}$ such that $\lambda=\vartheta \underline{m}_{S}^{R}$ for some $\vartheta: S \rightarrow R$ morphism in ${ }_{R} \mathcal{C}_{R}$.

Furthermore, if $R$ is a left $\otimes_{R^{-}}$generator for ${ }_{R} \mathcal{C}_{R}$ then the above assertions are also equivalent to

(ix) $-\otimes_{R} S:{ }_{R} \mathcal{C}_{R} \rightarrow{ }_{R} \mathcal{C}_{S}$ is a right adjoint of the forgetful functor $F:{ }_{R} \mathcal{C}_{S} \rightarrow{ }_{R} \mathcal{C}_{R}$ or, otherwise stated, $F$ is a Frobenius functor. 
So if an algebra extension $i: R \rightarrow S$ in $\mathcal{C}$ is Frobenius then $S$ has left and right dual objects in ${ }_{R} \mathcal{C}_{R}$. In the next sections we go further with this observation, by investigating when the existence of the dual object of $S$ in ${ }_{R} \mathcal{C}_{R}$ implies the existence of the dual object in $\mathcal{C}$, and vice-versa. The final aim is to characterize the Frobenius property of an algebra extensions $i: R \rightarrow S$ in terms given by the algebras $R$ and $S$. We will see that this is possible in the case when $R$ is Frobenius and separable.

\section{Monoidal Frobenius/separable algebra extensions versus monoidal Frobe- nius/separable functors}

We shall provide connections between the existence of the dual of an object $X$ in ${ }_{R} \mathcal{C}_{R}$ and the existence of the dual of the same object $X$, considered now as an object in $\mathcal{C}$ via the canonical forgetful functor $\mathfrak{U}:{ }_{R} \mathcal{C}_{R} \rightarrow \mathcal{C}$. In this direction, it is well-known that a strong monoidal functor preserves duals. Even more, it has been proved in [13, Theorem 2] that this remains true under the weaker hypothesis when in place of a strong monoidal functor we consider a Frobenius monoidal one. So a partial answer to our problem is offered by the case when $\mathfrak{U}$ is a Frobenius monoidal functor. This is why we start by giving a necessary and sufficient condition for which $\mathfrak{U}$ is Frobenius monoidal. As expected this reduces at the property of $R$ to be a Frobenius algebra.

Recall first from [53, Definition 6.1] and [13, Definition 1] the concept of (separable) Frobenius monoidal functor.

Definition 6.1. Let $(\mathcal{C}, \otimes, \underline{1})$ and $(\mathcal{D}, \square, \underline{I})$ be (strict) monoidal categories and $\mathfrak{F}: \mathcal{C} \rightarrow \mathcal{D}$ a functor.

(i) $\mathfrak{F}$ is called monoidal if there exist a family of morphisms

$$
\phi_{2}=\left(\phi_{X, Y}: \mathfrak{F}(X) \square \mathfrak{F}(Y) \rightarrow \mathfrak{F}(X \otimes Y)\right)_{X, Y \in \mathcal{C}},
$$

natural in $X$ and $Y$, and $\phi_{0}: \underline{I} \rightarrow \mathfrak{F}(\underline{1})$ morphism in $\mathcal{D}$ such that, for all $X, Y, Z \in \mathcal{C}$, the corresponding diagrams in (6.1) are commutative.

(ii) $\mathfrak{F}$ is called opmonoidal if there exist a family of morphisms

$$
\psi_{2}=\left(\psi_{X, Y}: \mathfrak{F}(X \otimes Y) \rightarrow \mathfrak{F}(X) \square \mathfrak{F}(Y)\right)_{X, Y \in \mathcal{C}},
$$

natural in $X$ and $Y$, and $\psi_{0}: \mathfrak{F}(\underline{1}) \rightarrow \underline{I}$ morphism in $\mathcal{D}$ such that, for all $X, Y, Z \in \mathcal{C}$, the corresponding diagrams in (6.1) are commutative.

$$
\begin{array}{r}
\mathfrak{F}(X) \square \mathfrak{F}(Y) \square \mathfrak{F}(Z) \frac{\phi_{X, Y} \square \mathrm{Id}_{\mathfrak{F}(Y)}}{\stackrel{\psi_{X, Y} \square \operatorname{Id}_{\mathfrak{F}(Z)}}{\longrightarrow}} \mathfrak{F}(X \otimes Y) \square \mathfrak{F}(Z), \\
\operatorname{Id}_{\mathfrak{F}(X)} \square \phi_{Y, Z}|\uparrow| \operatorname{Id}_{\mathfrak{F}(X)} \square \psi_{Y, Z}{ }_{X, Y, Z} \uparrow \mid \phi_{X \otimes Y, Z} \\
\mathfrak{F}(X) \square \mathfrak{F}(Y \otimes Z) \frac{\stackrel{\psi_{X, Y} \otimes Z}{\longleftarrow}}{\phi_{X, Y \otimes Z}} \mathfrak{F}(X \otimes Y \otimes Z)
\end{array}
$$




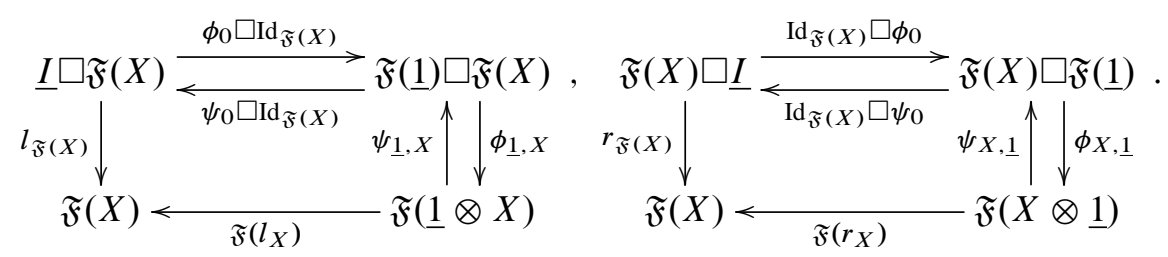

(iii) We call $\mathfrak{F}$ Frobenius monoidal if $\mathfrak{F}$ is equipped with a monoidal $\left(\phi_{2}, \phi_{0}\right)$ and comonoidal $\left(\psi_{2}, \psi_{0}\right)$ structure such that, for all $X, Y, Z \in \mathcal{C}$, the diagrams

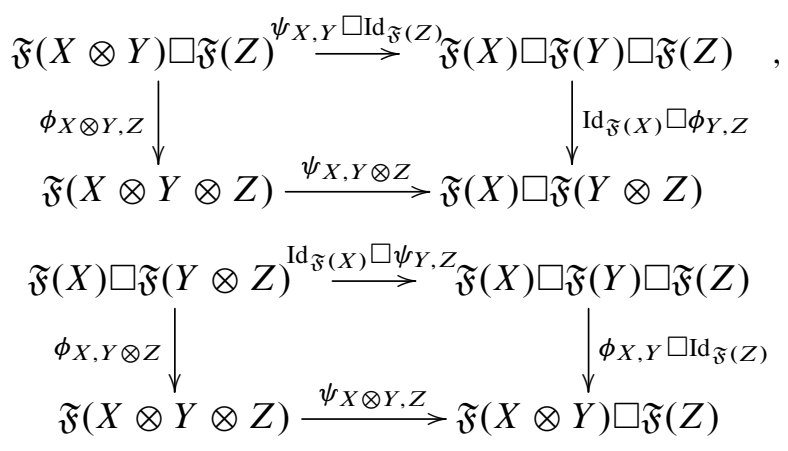

are commutative. If, furthermore, $\phi_{X, Y} \psi_{X, Y}=\operatorname{Id}_{\mathfrak{F}(X \otimes Y)}$, for all $X, Y \in \mathcal{C}$, we then say that $\mathfrak{F}$ is a separable Frobenius monoidal functor.

(iv) $\mathfrak{F}$ is called strong monoidal if it is a separable Frobenius monoidal functor, $\phi_{X, Y}$ is an isomorphism for all $X, Y \in \mathcal{C}$ (and thus $\phi_{X, Y}^{-1}=\psi_{X, Y}$ ), and $\phi_{0}$ and $\psi_{0}$ are inverses of each other.

We leave it to the reader to check that

$$
\phi_{2}=\left(\phi_{X, Y}=q_{X, Y}^{R}: X \otimes Y \rightarrow X \otimes_{R} Y\right)_{X, Y \in{ }_{R} \mathcal{C}_{R}}
$$

and

$$
\phi_{0}=\underline{\eta}_{R}: \underline{1} \rightarrow R
$$

define on the forgetful functor $\mathfrak{U}:{ }_{R} \mathcal{C}_{R} \rightarrow \mathcal{C}$ a monoidal structure. We refer to it as being the trivial monoidal structure of the functor $\mathfrak{U}$.

The next result gives the connection between the notions of Frobenius monoidal functor and Frobenius monoidal algebra. Its first statement generalizes [53, Lemmas $6.3 \& 6.4]$ while the second one is a completely new result; for short, it says that the opmonoidal structures of $\mathfrak{U}$ are uniquely determined by the Frobenius structures of $R$.

Theorem 6.2. If $R$ is an algebra in $\mathcal{C}$ then the forgetful functor $\mathfrak{U}:{ }_{R} \mathcal{C}_{R} \rightarrow \mathcal{C}$ endowed with the trivial monoidal structure $\left(q_{-,-}^{R}, \underline{\eta}_{R}\right)$ is Frobenius if and only if $R$ is a Frobenius algebra. Furthermore, if this is the case then the opmonoidal $\left(\psi_{2}, \psi_{0}\right)$ 
structure of $\mathfrak{U}$ is uniquely determined by a Frobenius structure of $R$, in the sense that there exists $(\vartheta, e)$ a Frobenius pair for $R$ such that $\psi_{0}=\vartheta$ and

$$
\psi_{2} q_{-,-}^{R}:=\left(\psi_{X, Y} q_{X, Y}^{R}\right)_{X, Y \in \epsilon_{R} \mathcal{C}_{R}}=\left(\left(v_{X}^{R} \otimes \mu_{Y}^{R}\right)\left(\operatorname{Id}_{X} \otimes e \otimes \operatorname{Id}_{Y}\right)\right)_{X, Y \in \in_{R} \mathcal{C}_{R}} .
$$

Proof. Assume that the functor $\mathfrak{U}$ admits a Frobenius monoidal structure. Then by [13, Corollary 5] $\mathfrak{U}$ carries Frobenius algebras to Frobenius algebras. Since $R$ is a Frobenius algebra in ${ }_{R} \mathcal{C}_{R}$ (as it is the unit object of a monoidal category) we get that $R$ is a Frobenius algebra in $\mathcal{C}$, as needed. Note that an alternative proof for this implication can be obtained from [53, Lemma 6.3]. It has the advantage that we can obtain the coalgebra structure $(\Delta, \varepsilon)$ of $R$ in $\mathcal{C}$ as follows. If $\left(\psi_{2}, \psi\right)$ is the comonoidal structure of $\mathfrak{U}$ that together with the trivial monoidal structure gives on $\mathfrak{U}$ a Frobenius monoidal functor structure then $\Delta=\psi_{R, R} \Upsilon_{R}^{-1}: R \rightarrow R \otimes R$ and $\varepsilon=\psi_{0}: R \rightarrow \underline{1}$, respectively.

Conversely, suppose that $R$ is a Frobenius algebra in $\mathcal{C}$ and let $(\vartheta, e)$ be a Frobenius system for $R$. For $X, Y \in{ }_{R} \mathcal{C}_{R}$ denote by $\psi_{X, Y}$ the morphism in $\mathcal{C}$ uniquely determined by $\psi_{X, Y} q_{X, Y}^{R}=\left(v_{X}^{R} \otimes \mu_{Y}^{R}\right)\left(\operatorname{Id}_{X} \otimes e \otimes \operatorname{Id}_{Y}\right)$. Note that $\left(v_{X}^{R} \otimes \mu_{Y}^{R}\right)\left(\operatorname{Id}_{X} \otimes e \otimes \operatorname{Id}_{Y}\right): X \otimes Y \rightarrow X \otimes Y$ fits in the universal property of the cooequalizer

$$
X \otimes R \otimes Y \underset{\operatorname{Id}_{X} \otimes \mu_{Y}^{R}}{\stackrel{v_{X}^{R} \otimes \mathrm{Id}_{Y}}{\longrightarrow}} X \otimes Y \stackrel{q_{X, Y}^{R}}{\longrightarrow} X \otimes_{R} Y
$$

since $\left(\underline{m}_{R} \otimes \operatorname{Id}_{R}\right)\left(\operatorname{Id}_{R} \otimes e\right)=\left(\operatorname{Id}_{R} \otimes \underline{m}_{R}\right)\left(e \otimes \operatorname{Id}_{R}\right)$. Now a simple computation shows that the diagrams in (6.1) and (6.2) are commutative, and so $\mathfrak{U}$ is a Frobenius monoidal functor.

It remains to prove that if $\left(q_{-,-}^{R}, \underline{\eta}_{R}, \psi_{2}, \psi_{0}\right)$ defines a Frobenius monoidal functor structure on $\mathfrak{U}$ then there exists $(\vartheta, e)$ a Frobenius system for $R$ such that the comonoidal structure $\left(\psi_{2}, \psi_{0}\right)$ of $\mathfrak{U}$ is completely determined by it, in the sense that $\psi_{0}=\vartheta$ and $\psi_{2}$ is defined by (6.3). To this end we first show that $\psi_{X, Y}$ is $R$-bilinear for any $X, Y \in{ }_{R} \mathcal{C}_{R}$.

Indeed, if we take $Z=R$ in the first diagram of (6.2) we then have

$$
\left(\operatorname{Id}_{X} \otimes q_{Y, R}^{R}\right)\left(\psi_{X, Y} \otimes \operatorname{Id}_{R}\right)=\psi_{X, Y \otimes_{R} R} \Sigma_{X, Y, R}^{\prime} q_{X \otimes_{R} Y, R}^{R}
$$

Composing both sides of the above equality to the right with $\operatorname{Id}_{X} \otimes \Upsilon_{Y}$, and taking into consideration that the diagram

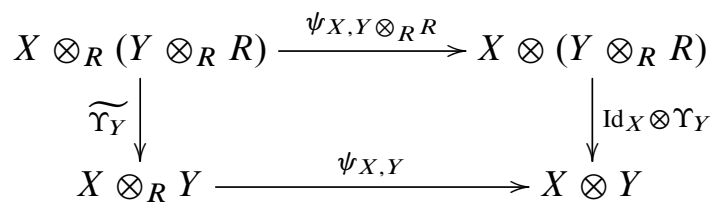


is commutative $\left(\psi_{X,-}\right.$ is natural and $\Upsilon_{Y}: Y \otimes_{R} R \rightarrow Y$ is a morphism in $\left.{ }_{R} \mathcal{C}_{R}\right)$ we deduce that

$$
\begin{aligned}
\left(\operatorname{Id}_{X} \otimes v_{Y}^{R}\right)\left(\psi_{X, Y} q_{X, Y}^{R} \otimes \operatorname{Id}_{R}\right) & =\psi_{X, Y} \widetilde{\Upsilon_{Y}} q_{X, Y \otimes_{R} R}^{R}\left(\operatorname{Id}_{X} \otimes q_{Y, R}^{R}\right) \\
& =\psi_{X, Y} q_{X, Y}^{R}\left(\operatorname{Id}_{X} \otimes v_{Y}^{R}\right) \\
& =\psi_{X, Y} v_{X \otimes_{R} Y}^{R}\left(q_{X, Y}^{R} \otimes \operatorname{Id}_{R}\right) .
\end{aligned}
$$

Hence $\left(\operatorname{Id}_{X} \otimes v_{Y}^{R}\right)\left(\psi_{X, Y} \otimes \operatorname{Id}_{R}\right)=\psi_{X, Y} v_{X \otimes_{R} Y}^{R}$, and this shows that $\psi_{X, Y}$ is right $R$-linear in $\mathcal{C}$.

Likewise, if we take $X=R$ in the second diagram in (6.2) and use the fact that $\left(\Upsilon_{Y}^{\prime} \otimes \operatorname{Id}_{Z}\right) \psi_{R \otimes_{R} Y, Z}=\psi_{Y, Z} \widehat{\Upsilon_{Y}^{\prime}}\left(\psi_{-, Z}\right.$ is natural and $\Upsilon_{Y}^{\prime}: R \otimes_{R} Y \rightarrow Y$ is a morphism in ${ }_{R} \mathcal{C}_{R}$ ), by a computation similar to the one above we get that $\psi_{Y, Z}$ is left $R$-linear, for all $Y, Z \in{ }_{R} \mathcal{C}_{R}$. Therefore $\psi_{2}$ is defined by a family of $R$-bilinear morphisms in $\mathcal{C}$, as desired.

Now, if we define $\Delta:=\psi_{R, R} \Upsilon_{R}^{-1}=\psi_{R, R} \Upsilon_{R}^{\prime-1}: R \rightarrow R \otimes R$ it then follows that $\Delta$ is $R$-bilinear. So $\Delta$ is uniquely determined by $e:=\Delta \underline{\eta}_{R}: \underline{1} \rightarrow R \otimes R$, a morphism in $\mathcal{C}$ which has the property that

$$
\left(\underline{m}_{R} \otimes \operatorname{Id}_{R}\right)\left(\operatorname{Id}_{R} \otimes e\right)=\left(\operatorname{Id}_{R} \otimes \underline{m}_{R}\right)\left(e \otimes \operatorname{Id}_{R}\right) .
$$

We claim that $e$ determines completely $\psi_{2}$. Indeed, if we take $Y=R$ in the first diagram of (6.2) and use the naturalness of $\psi_{X,-}$ for the morphism $\Upsilon_{Z}^{\prime}: R \otimes_{R} Z \rightarrow Z$ in ${ }_{R} \mathcal{C}_{R}$ we obtain

$$
\begin{aligned}
\left(\operatorname{Id}_{X} \otimes \mu_{Z}^{R}\right)\left(\psi_{X, R} q_{X, R}^{R} \otimes \operatorname{Id}_{Z}\right) & =\left(\operatorname{Id}_{X} \otimes \Upsilon_{Z}^{\prime}\right)\left(\operatorname{Id}_{X} \otimes q_{R, Z}^{R}\right)\left(\psi_{X, R} q_{X, R}^{R} \otimes \operatorname{Id}_{Z}\right) \\
& =\psi_{X, Z} \widetilde{\Upsilon_{Z}^{\prime}} q_{X, R \otimes_{R} Z}^{R}\left(\operatorname{Id}_{X} \otimes q_{R, Z}^{R}\right) \\
& =\psi_{X, Z} q_{X, Z}^{R}\left(\operatorname{Id}_{X} \otimes \mu_{Z}^{R}\right) .
\end{aligned}
$$

Composing both sides of the latter equality to the right with $\operatorname{Id}_{X} \otimes \underline{\eta}_{R} \otimes \operatorname{Id}_{Z}$ we deduce that $\psi_{X, Z} q_{X, Z}^{R}=\left(\operatorname{Id}_{X} \otimes \mu_{Z}^{R}\right)\left(\psi_{X, R} \Upsilon_{X}^{-1} \otimes \operatorname{Id}_{Z}\right)$, for all $X, Z \in{ }_{R} \mathcal{C}_{R}$.

Similarly, take $Y=R$ in the second diagram of (6.2) and use

$$
\left(\Upsilon_{X} \otimes \operatorname{Id}_{Z}\right) \psi_{X \otimes_{R} R, Z}=\psi_{X, Z} \widehat{\Upsilon_{X}}
$$

to get

$$
\psi_{X, Z} q_{X, Z}^{R}=\left(v_{X}^{R} \otimes \operatorname{Id}_{Z}\right)\left(\operatorname{Id}_{X} \otimes \psi_{R, Z} \Upsilon_{Z}^{\prime-1}\right),
$$

for all $X, Z \in{ }_{R} \mathcal{C}_{R}$. Combining these two equalities we get

$$
\begin{aligned}
\psi_{X, Z} q_{X, Z}^{R} & =\left(v_{X}^{R} \otimes \operatorname{Id}_{Z}\right)\left(\operatorname{Id}_{X} \otimes \psi_{R, Z} q_{R, Z}^{R}\left(\underline{\eta}_{R} \otimes \operatorname{Id}_{Z}\right)\right) \\
& =\left(v_{X}^{R} \otimes \operatorname{Id}_{Z}\right)\left(\operatorname{Id}_{X} \otimes\left(\operatorname{Id}_{R} \otimes \mu_{Z}^{R}\right)\left(\psi_{R, R} \Upsilon_{R}^{-1} \underline{\eta}_{R} \otimes \operatorname{Id}_{Z}\right)\right) \\
& =\left(v_{X}^{R} \otimes \operatorname{Id}_{Z}\right)\left(\operatorname{Id}_{X} \otimes\left(\operatorname{Id}_{R} \otimes \mu_{Z}^{R}\right)\left(e \otimes \operatorname{Id}_{Z}\right)\right)
\end{aligned}
$$




$$
=\left(v_{X}^{R} \otimes \mu_{Z}^{R}\right)\left(\operatorname{Id}_{X} \otimes e \otimes \operatorname{Id}_{Z}\right),
$$

for all $X, Z \in{ }_{R} \mathcal{C}_{R}$, as claimed. Furthermore, it is immediate now that the commutativity of the two square diagrams in (6.1) is equivalent to $\left(\psi_{0} \otimes \operatorname{Id}_{R}\right) e=\underline{\eta}_{R}$ and $\left(\operatorname{Id}_{R} \otimes \psi_{0}\right) e=\underline{\eta}_{R}$, respectively. In conclusion, $\left(\vartheta=\psi_{0}, e=\psi_{R, R} \Upsilon_{R}^{-1} \eta_{R}\right)$ is a Frobenius system for $R$ and determines completely the opmonoidal structure $\left(\psi_{2}, \psi_{0}\right)$ of $\mathfrak{U}$. This finishes the proof.

Corollary 6.3. If $i: R \rightarrow S$ is a Frobenius algebra extension with $R$ a Frobenius algebra in $\mathcal{C}$ then $S$ is a Frobenius algebra in $\mathcal{C}$, too.

Proof. Since $R$ is a Frobenius algebra in $\mathcal{C}$ we have that the forgetful functor $\mathfrak{U}:{ }_{R} \mathcal{C}_{R} \rightarrow \mathcal{C}$ is Frobenius monoidal, so it carries Frobenius algebras in ${ }_{R} \mathcal{C}_{R}$ to Frobenius algebras in $\mathcal{C}$. The fact that $i: R \rightarrow S$ is a Frobenius algebra extension is equivalent to the fact that $S$ is a Frobenius algebra in ${ }_{R} \mathcal{C}_{R}$, cf. Proposition 4.8. Thus $S=\mathfrak{U}(S)$ is a Frobenius algebra in $\mathcal{C}$.

Corollary 6.4. Let $R$ be a Frobenius algebra in $\mathcal{C}$. If $X \in{ }_{R} \mathcal{C}_{R}$ admits a (left) right dual object in ${ }_{R} \mathcal{C}_{R}$ then it admits a (left) right dual object in $\mathcal{C}$, too.

Proof. Follows from the fact that a Frobenius monoidal functor behaves well with respect to dual objects, see [13, Theorem 2]. Actually, if $(\rho, \lambda): Y \dashv X$ is an adjunction in ${ }_{R} \mathcal{C}_{R}$ then

$$
\rho^{\prime}:=\left(\underline{1} \stackrel{\underline{\eta}_{R}}{\longrightarrow} R \stackrel{\rho}{\longrightarrow} X \otimes_{R} Y \stackrel{\psi_{X, Y}}{\longrightarrow} X \otimes Y\right)
$$

and

$$
\lambda^{\prime}=\left(X \otimes Y \stackrel{q_{X, Y}^{R}}{\longrightarrow} X \otimes_{R} Y \stackrel{\lambda}{\longrightarrow} R \stackrel{\psi_{0}}{\longrightarrow} \underline{1}\right)
$$

defines an adjunction $\left(\rho^{\prime}, \lambda^{\prime}\right): Y \dashv X$ in $\mathcal{C}$, where $\left(\psi_{2}, \psi_{0}\right)$ is uniquely determined by a Frobenius system $(\vartheta, e)$ of $R$ as in Theorem 6.2.

Corollary 6.5. Under the hypothesis and notions of Theorem 6.2 we have that $\mathfrak{U}:{ }_{R} \mathcal{C}_{R} \rightarrow \mathcal{C}$ is a separable Frobenius monoidal functor if and only if $R$ is a Frobenius separable algebra in $\mathcal{C}$.

Proof. It is obvious. Note only that the condition $\phi_{X, Y} \psi_{X, Y}=\operatorname{Id}_{X \otimes_{R} Y}$, for all $X, Y \in{ }_{R} \mathcal{C}_{R}$, is equivalent to $\underline{m}_{R} e=\underline{\eta}_{R}$. 


\section{Frobenius algebra extensions in sovereign monoidal categories}

Let $i: R \rightarrow S$ be a Frobenius algebra extension such that $R$ is a Frobenius algebra in $\mathcal{C}$. Thus $S$ is a Frobenius algebra as well, cf. Corollary 6.3. Our next goal is to extend this result. Namely, we will prove that when $R$ is, moreover, separable then $i: R \rightarrow S$ is Frobenius if and only if $S$ is a Frobenius algebra and an extra condition is fulfilled (see (7.1) below). In the case when $\mathcal{C}$ is a sovereign monoidal category this extra condition says that the restriction at $R$ of the Nakayama automorphism of $S$ coincides with the Nakayama automorphism of $R$.

To this end we need first a kind of converse for Corollary 6.4, and this forces us to assume that $R$ is separable, too. Note that a similar result was proved in [55] but for a special class of Frobenius algebras. Namely, for those Frobenius algebras $A$ in $k$-linear monoidal categories that satisfy, in addition, $\underline{m}_{A} \underline{\Delta}_{A}=d_{A} \operatorname{Id}_{A}$ and $\underline{\varepsilon}_{A} \underline{\eta}_{A}=d_{A} \underline{1}$, for a certain non-zero scalar $d_{A}$. (Here we made use of the characterization of a Frobenius algebra presented in Theorem 5.1 (iv).) Our result below is more general than the one in [55, Lemma 5.5]. On one hand, our adjunction is independent on $d_{A}\left(d_{A}\right.$ does not appear in the right hand side of (5.2) or (5.4)), and we work in an arbitrary monoidal category. On the other hand, there are situations where the class of Frobenius algebras that are separable so that the Frobenius system and the separability morphism coincide (we refer at the condition $\underline{m}_{A} \underline{\Delta}_{A}=d_{A} \mathrm{Id}_{A}$ ) is less general than the class considered here: Frobenius algebras that are separable, too (for a concrete example see the end of $\S 3$ in [47]). Also, the second condition $\left(\underline{\varepsilon}_{A} \underline{\eta}_{A}=d_{A} \underline{1}\right)$ is quite restrictive as $\underline{\varepsilon}_{A}$ is given by the Frobenius morphism; for example, a finite dimensional Hopf algebra is always Frobenius with Frobenius morphism an integral $\lambda$ on $H$, and the condition $\lambda(1)=d 1$, for some non-zero scalar $d$, is equivalent to $H$ cosemisimple. Finally, our next result generalizes as well [19, Proposition 5.15] which says that for a swap-commutative special Frobenius algebra $A$ the category $\mathcal{C}_{A}$ is rigid as long as $\mathcal{C}$ is so.

Proposition 7.1. Consider $R$ a Frobenius separable algebra in $\mathcal{C}$ and let $(\vartheta, e)$ be a Frobenius pair for $R, \alpha: \underline{1} \rightarrow R$ as in Proposition 4.10 and $(\rho, \lambda): Y \dashv X$ an adjunction in $\mathcal{C}$.

(i) If $Y$ has an $R$-bimodule structure in $\mathcal{C}$ then $X$ has an $R$-bimodule structure in $\mathcal{C}$, too, and via these structures the morphisms

$$
\begin{aligned}
& \rho_{0}=\left(R \stackrel{\rho \otimes \mathrm{Id}_{R}}{\longrightarrow} X \otimes Y \otimes R\right. \stackrel{\operatorname{Id}_{X} \otimes \alpha \otimes \operatorname{Id}_{Y} \otimes R}{\longrightarrow} X \otimes R \otimes Y \otimes R \\
&\left.\stackrel{\operatorname{Id}_{X} \otimes \mu_{Y}^{R} \nu_{Y}^{R}}{\longrightarrow} X \otimes Y \stackrel{q_{X, Y}^{R}}{\longrightarrow} X \otimes_{R} Y\right)
\end{aligned}
$$

and $\lambda_{0}: Y \otimes_{R} X \rightarrow R$ uniquely determined by

$$
\begin{aligned}
& \lambda_{0} q_{Y, X}^{R}=\left(Y \otimes X \stackrel{e \otimes \operatorname{Id}_{Y} \otimes X}{\longrightarrow} R \otimes R \otimes Y \otimes X\right. \\
& \left.\stackrel{\operatorname{Id}_{R} \otimes \mu_{Y}^{R} \otimes \operatorname{Id}_{X}}{\longrightarrow} R \otimes Y \otimes X \stackrel{\operatorname{Id}_{R} \otimes \lambda}{\longrightarrow} R\right)
\end{aligned}
$$

define an adjunction $\left(\rho_{0}, \lambda_{0}\right): Y \dashv X$ in ${ }_{R} \mathcal{C}_{R}$. 
(ii) Similarly, if $X$ admits an $R$-bimodule structure in $\mathcal{C}$ then $Y$ admits an $R$ bimodule structure in $\mathcal{C}$ as well, and via these structures the morphisms

$$
\begin{aligned}
& \rho^{0}=\left(R \stackrel{\operatorname{Id}_{R} \otimes \rho}{\longrightarrow} R \otimes X \otimes Y \stackrel{\operatorname{Id}_{R} \otimes X \otimes \alpha \otimes \mathrm{Id}_{Y}}{\longrightarrow} R \otimes X \otimes R \otimes Y\right. \\
& \left.\stackrel{\mu_{X}^{R} v_{X}^{R} \otimes \operatorname{Id}_{Y}}{\longrightarrow} X \otimes Y \stackrel{q_{X, Y}^{R}}{\longrightarrow} X \otimes_{R} Y\right)
\end{aligned}
$$

and $\lambda^{0}: Y \otimes_{R} X \rightarrow R$ uniquely determined by

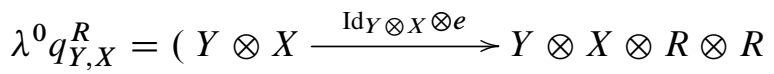

$$
\begin{aligned}
& \left.\stackrel{\operatorname{Id}_{Y} \otimes v_{X}^{R} \otimes \operatorname{Id}_{R}}{\longrightarrow} Y \otimes X \otimes R \stackrel{\lambda \otimes \operatorname{Id}_{R}}{\longrightarrow} R\right)
\end{aligned}
$$

define an adjunction $\left(\rho^{0}, \lambda^{0}\right): Y \dashv X$ in ${ }_{R} \mathcal{C}_{R}$.

Proof. We prove only (i). The proof of (ii) is similar, and can be obtained from that of (i) by reversing the structures, and so the diagrammatic computations as well, through a mirror. In other words the statement (ii) is the statement (i) for the monoidal category $\overline{\mathcal{C}}$, where $\overline{\mathcal{C}}$ is the reverse monoidal category associated to $\mathcal{C}$.

(i) From now on, for $Z$ a left $R$-module in $\mathcal{C}$ we denote by $\frac{R Z}{Z}$ the structure morphism $\mu_{Z}^{R}$. Similarly, if $Z$ is a right $R$-module in $\mathcal{C}$ then the structure morphism $v_{Z}^{R}$ will be denoted by $\frac{Z R}{Z}$. Also, for the Frobenius separable system $(e, \vartheta, \alpha)$ of $R$, and respectively for the morphisms of the adjunction $(\rho, \lambda): Y \dashv X$, we will use the following diagrammatic notations,

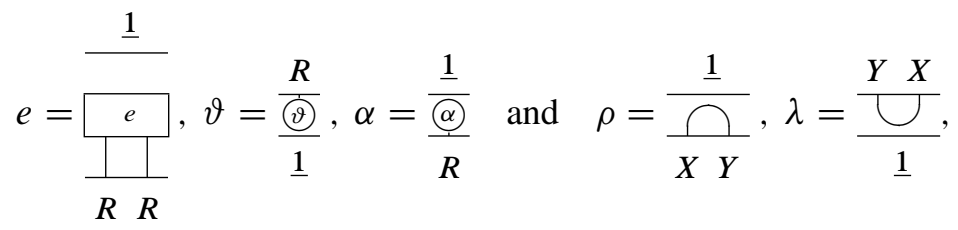

respectively. With their help define the morphisms $\delta: R \rightarrow X \otimes Y$ and $\gamma: Y \otimes X \rightarrow R$ given by

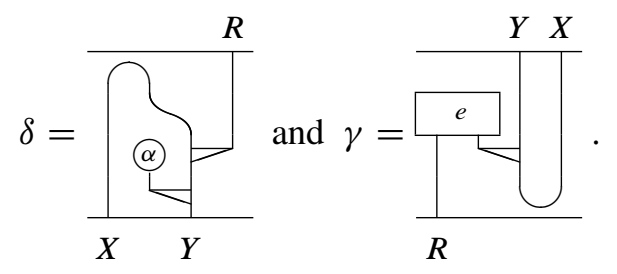


Furthermore, if $Y$ is an $R$-bimodule in $\mathcal{C}$ we then define on ${ }_{X}^{X}$ the following left and right $R$-actions,
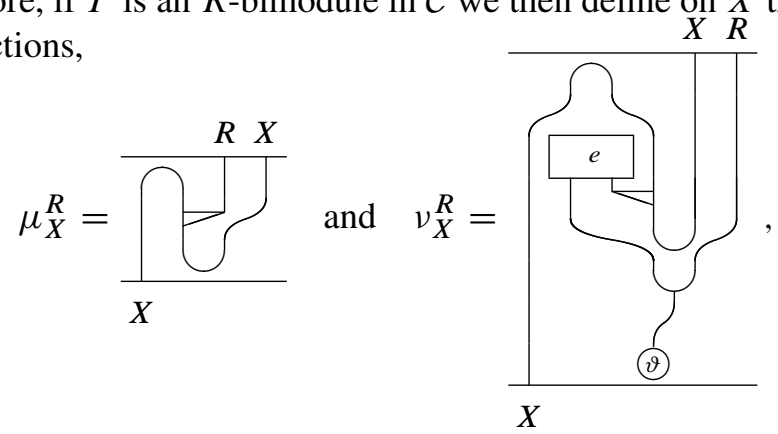

respectively. In fact, it can be easily checked that $\mu_{X}^{R}$ yields a left $R$-module structure on $X$ and since
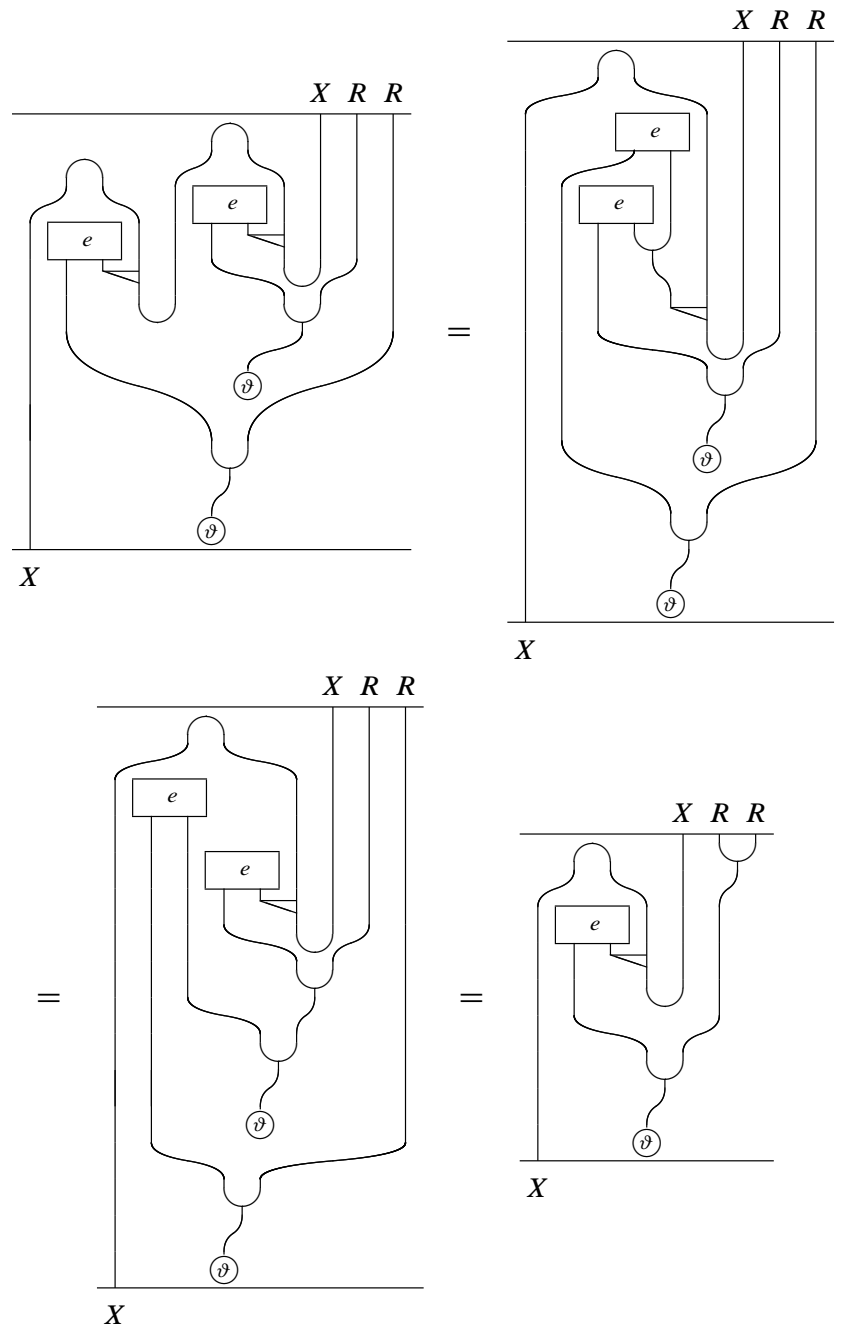
it follows that $X$ is a right $R$-module in $\mathcal{C}$ via $v_{X}^{R}$, too. It is, moreover, an $R$-bimodule because of the defining properties of an adjunction and since $Y$ is so, we leave to the reader the verification of these details.

It is clear that $\rho_{0}=\delta q_{X, Y}^{R}$. A simple computation based on the properties of an adjunction and on the fact that $Y$ is an $R$-bimodule ensures us that $\gamma$ fits in the universal property of the coequalizer

$$
Y \otimes R \otimes X \underset{\operatorname{Id}_{Y} \otimes \mu_{X}^{R}}{\stackrel{v_{Y}^{R} \otimes \operatorname{Id} X}{\longrightarrow}} Y \otimes X \stackrel{q_{Y, X}^{R}}{\longrightarrow} Y \otimes_{R} X .
$$

Thus there is a unique morphism $\lambda_{0}: Y \otimes_{R} X \rightarrow R$ such that $\lambda_{0} q_{Y, X}^{R}=\gamma$.

The morphisms $\rho_{0}$ and $\lambda_{0}$ constructed above are morphisms in ${ }_{R} \mathcal{C}_{R}$. For instance,

$$
\begin{aligned}
\lambda_{0} \mu_{Y \otimes_{R} X}^{R}\left(\operatorname{Id}_{R} \otimes q_{Y, X}^{R}\right) & =\lambda_{0} q_{Y, X}^{R}\left(\mu_{Y}^{R} \otimes \operatorname{Id}_{X}\right)=\gamma\left(\mu_{Y}^{R} \otimes \operatorname{Id}_{X}\right) \\
& =\frac{{ }^{R Y X}}{R}
\end{aligned}
$$

and this shows that $\lambda_{0}$ is left $R$-linear in $\mathcal{C}$. Similarly,

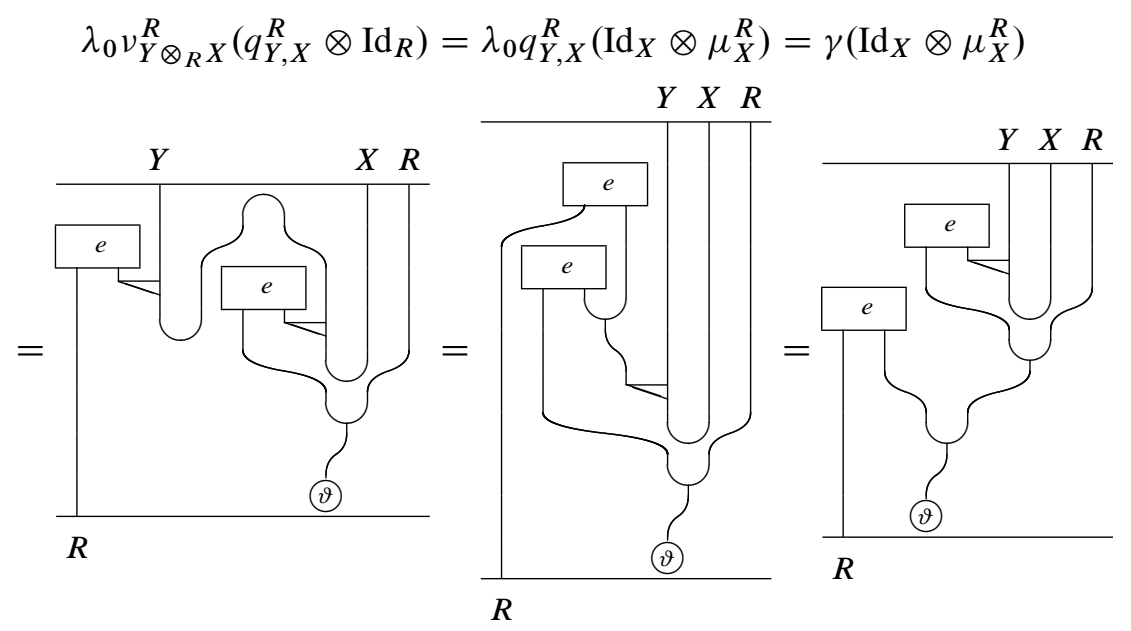




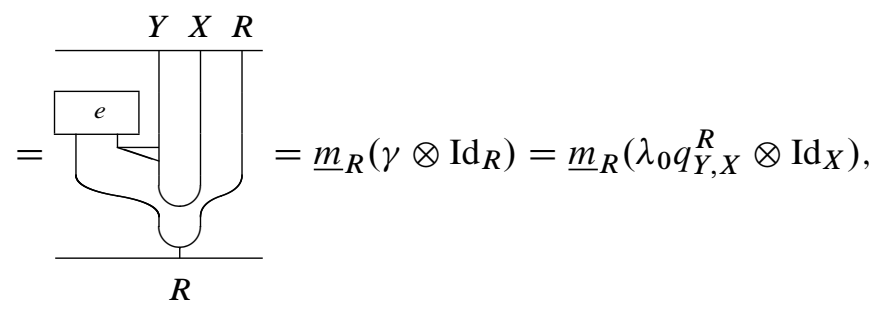

and so $\lambda_{0}$ is right $R$-linear, too. In a similar manner we can show that $\rho_{0}$ is $R$-bilinear, once more we leave the details to the reader.

It remains to prove that $\left(\rho_{0}, \lambda_{0}\right): Y \dashv X$ is an adjunction in ${ }_{R} \mathcal{C}_{R}$. Towards this end we compute

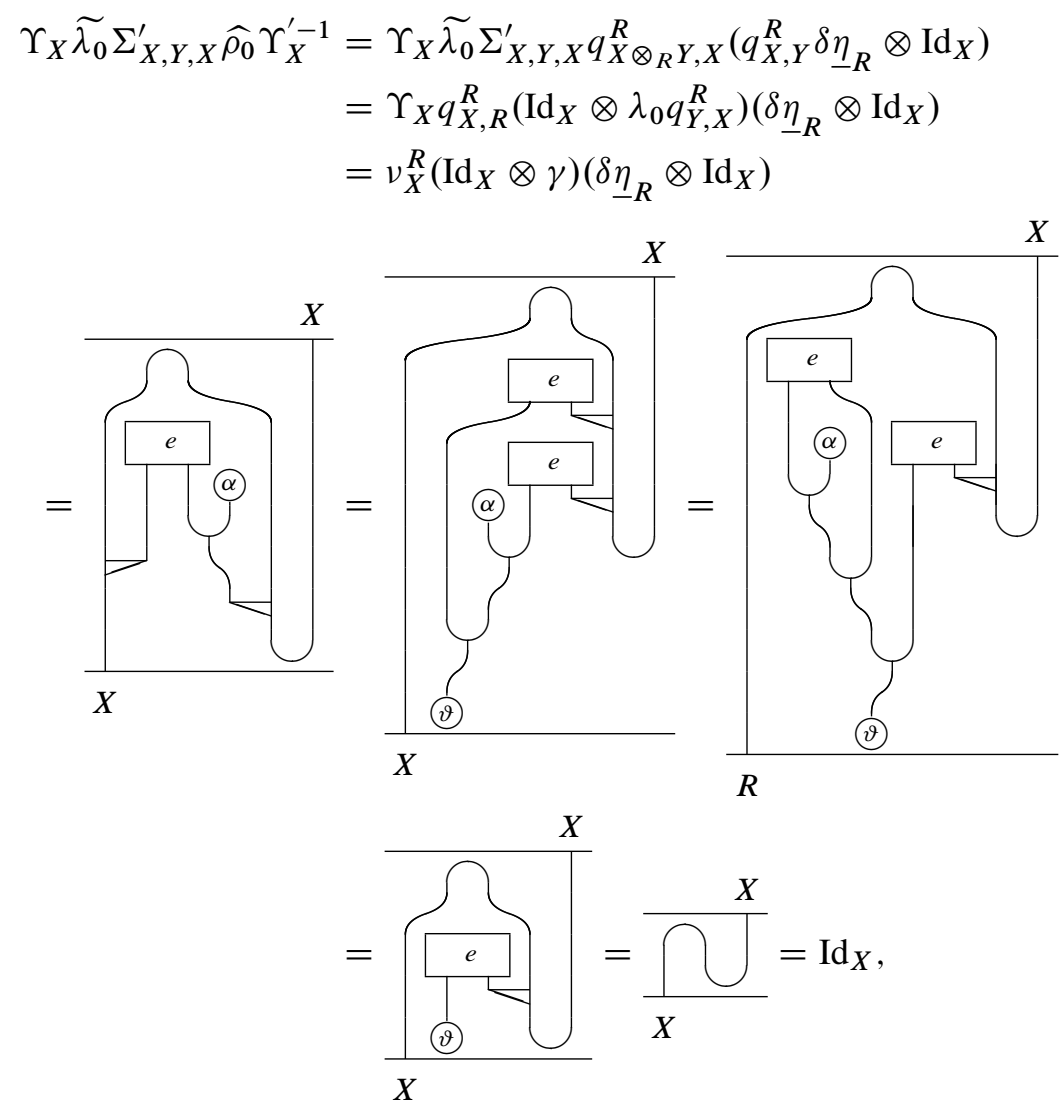

as required. Analogously we have $\Upsilon_{Y}^{\prime} \widehat{\lambda_{0}} \Gamma_{Y, X, Y}^{\prime} \widetilde{\rho_{0}} \Upsilon_{Y}^{-1}=\operatorname{Id}_{Y}$. This ends the proof.

Corollary 7.2. Let $R$ be a Frobenius separable algebra and $X$ an $R$-bimodule in $\mathcal{C}$. Then $X$ admits a (left) right dual in ${ }_{R} \mathcal{C}_{R}$ if and only if $X$ admits a (left) right dual in $\mathcal{C}$. 
Proof. The if part follows from Corollary 6.4. For the converse, if $X$ has a (left) right dual $\left(X^{*}\right)^{*} X$ then we have an adjunction $\left(\left(\operatorname{ev}_{X}, \operatorname{coev}_{X}\right): X^{*} \dashv X\right)$ $\left(\mathrm{ev}_{X}^{\prime}, \operatorname{coev}_{X}^{\prime}\right): X \dashv^{*} X$ in $\mathcal{C}$. Since $X$ is an $R$-bimodule in $\mathcal{C}$ it follows that we have an adjunction $\left(\left(\left(\operatorname{ev}_{X}\right)^{0},\left(\operatorname{coev}_{X}\right)^{0}\right): X^{*} \dashv X\right)\left(\left(\operatorname{ev}_{X}^{\prime}\right)_{0},\left(\operatorname{coev}_{X}^{\prime}\right)_{0}\right): X \dashv^{*} X$ in ${ }_{R} \mathcal{C}_{R}$, cf. Proposition 7.1. This finishes the proof.

We are now in position to prove one of the main results of the paper.

Theorem 7.3. Let $R$ be a Frobenius separable algebra and $i: R \rightarrow S$ an algebra extension in $\mathcal{C}$. Then $i: R \rightarrow S$ is Frobenius if and only if $S$ is a Frobenius algebra in $\mathcal{C}$ and the following equality holds

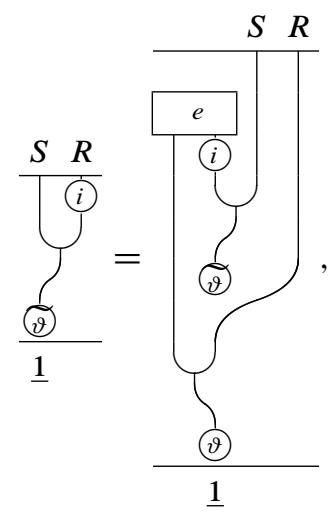

where $(\vartheta, e)$ is a Frobenius pair for $R$ and $(\widetilde{\vartheta}, \widetilde{e})$ is a Frobenius pair for $S$, respectively.

Proof. If $i: R \rightarrow S$ is a Frobenius algebra extension with $R$ a Frobenius separable algebra in $\mathcal{C}$ then we have seen that $S$ is a Frobenius algebra in $\mathcal{C}$ (Corollary 6.3). Furthermore, if $\vartheta: R \rightarrow \underline{1}$ and $\vartheta^{\prime}: S \rightarrow R$ are the Frobenius morphisms corresponding to $R$ and to extension $i: R \rightarrow S$, respectively, then $S$ is Frobenius with Frobenius morphism given by $\widetilde{\vartheta}=\vartheta \vartheta^{\prime}$. Consider now $e$ the Casimir morphism of the Frobenius algebra $R$, corresponding to $\vartheta$. Since $\vartheta^{\prime}$ is a morphism in ${ }_{R} \mathcal{C}_{R}$ we compute that

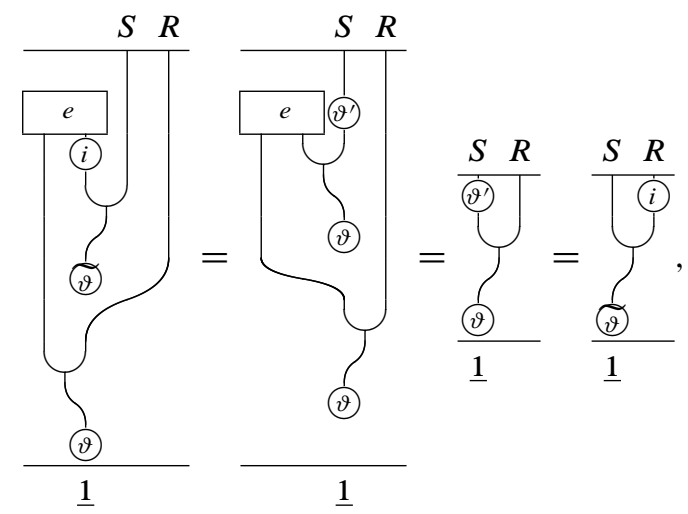


as wished. Conversely, assume that $S$ is a Frobenius algebra in $\mathcal{C}$ and let $(\widetilde{\vartheta}, \widetilde{e})$ be a Frobenius pair for it. By Theorem 5.1 we know that $S$ admits a right dual object ${ }^{*} S$ in $\mathcal{C}$ and, moreover, the morphism $\Phi: S \rightarrow{ }^{*} S$ given by

$$
\Phi=\frac{\underbrace{S}_{{ }^{*} S}}{\overbrace{\circledast}}
$$

is an isomorphism of left $S$-modules in $\mathcal{C}$. This comes out explicitly as



and implies

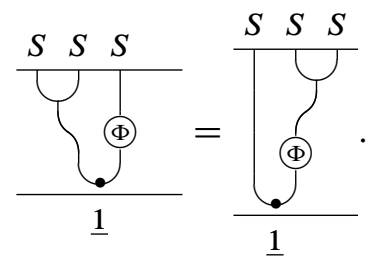

By Corollary 7.2 we have that ${ }^{*} S$ is an $R$-bimodule in $\mathcal{C}$ via the structure morphisms given by

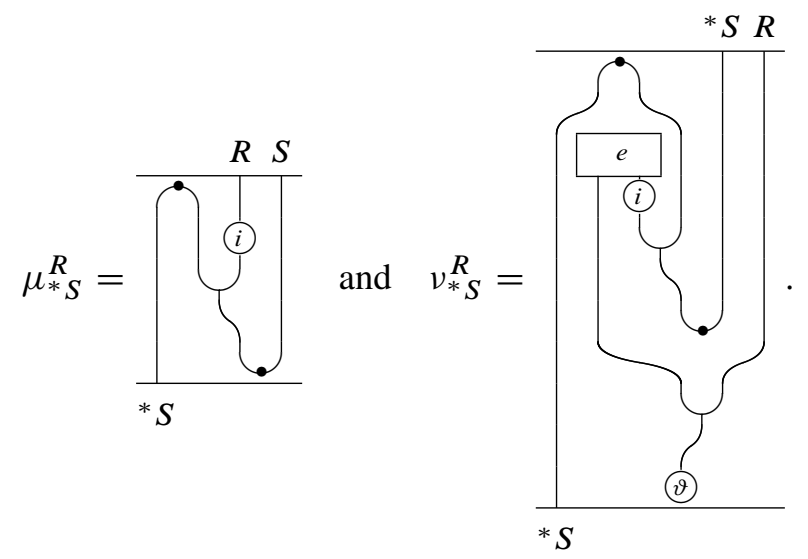

Observe that $\mu_{* S}^{R}$ is nothing that the restriction at $R$ of the canonical left $S$-module structure on ${ }^{*} S$ via the algebra morphism $i: R \rightarrow S$. Thus $\Phi$ is left $R$-linear. $\Phi$ is 
also right $R$-linear since with the help of (7.1) we compute that
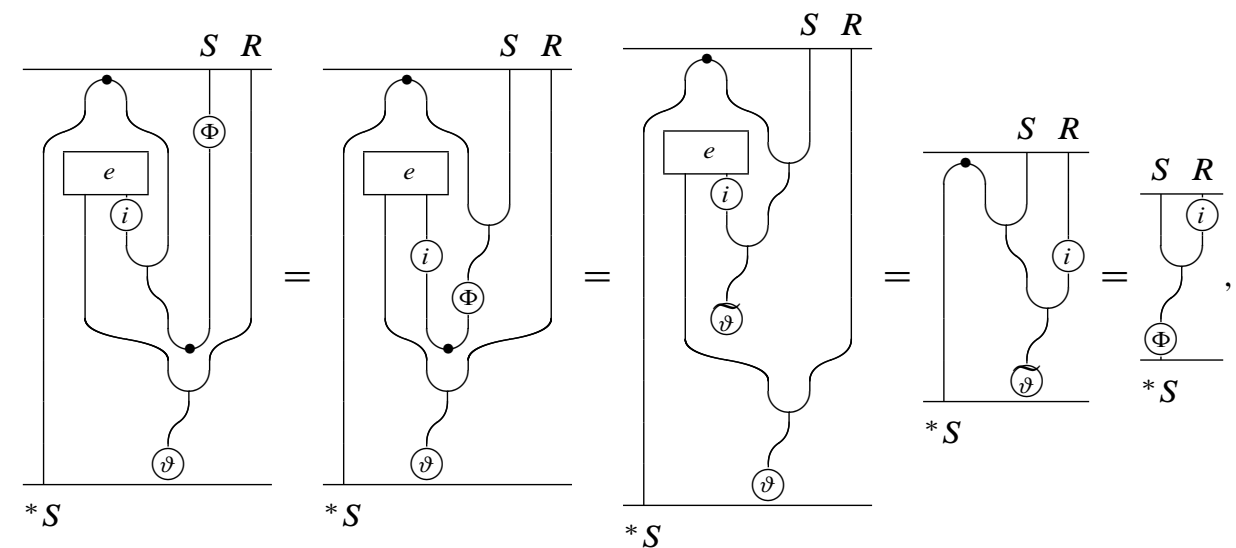

as needed. Therefore $\Phi: S \rightarrow{ }^{*} S$ is an isomorphism in ${ }_{R} \mathcal{C}_{R}$ and a left $S$-module morphism in $\mathcal{C}$. We show next that $\Phi$ is a left $S$-linear morphism in ${ }_{R} \mathcal{C}_{R}$ between $S$ and ${ }^{*} S$, where ${ }^{*} S$ is considered this time as the right dual of $S$ in ${ }_{R} \mathcal{C}_{R}$. According to Corollary 5.2 this will end the proof.

Indeed, recall that ${ }^{*} S$ is also a right dual for $S$ in ${ }_{R} \mathcal{C}_{R}$ via the evaluation and coevaluation morphisms in ${ }_{R} \mathcal{C}_{R}$ completely determined by

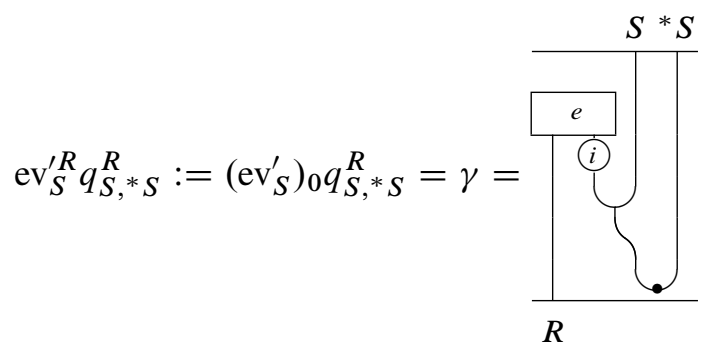

and

$$
\operatorname{coev}_{S}^{\prime R}:=\left(\operatorname{covv}_{S}^{\prime}\right)_{0}=q_{* S, S}^{R} \circ \delta=q_{* S, S}^{R} \circ \overbrace{{ }^{*} S}^{(\infty)} \int_{S}^{i},
$$

respectively. Thus, if $\mu_{* S}^{R, S}$ denotes the left $S$-module structure of ${ }^{*} S$ in ${ }_{R} \mathcal{C}_{R}$ then

$$
\begin{aligned}
& \mu_{* S}^{R, S} q_{S,{ }^{*} S}^{R}=\Upsilon_{* S} \widetilde{\operatorname{ev}_{S}^{\prime R}} \widetilde{\widetilde{m_{S}^{R}}} \widetilde{\Gamma_{S, S,{ }_{S}}^{\prime}} \Sigma^{\prime}{ }^{\prime} S, S, S \otimes_{R}{ }^{*} S^{\operatorname{coev}_{S}^{R}} \Upsilon_{S \otimes_{R}{ }^{*} S}^{\prime-1} q_{S,{ }^{*} S}^{R} \\
& =\Upsilon_{* S} \widetilde{\operatorname{ev}_{S}^{\prime R} \underline{m}_{S}^{R}} \widetilde{\Gamma_{S, S,{ }^{*} S}^{\prime}} \Sigma_{* S, S, S \otimes_{R}{ }^{*} S}^{\prime R} q^{R} \otimes_{R} S, S \otimes_{R}{ }^{*} S \\
& \left(q^{*} S, S=\operatorname{Id}_{S \otimes_{R}{ }^{*} S}\right)\left(\operatorname{Id}_{R} \otimes q_{S,{ }^{*} S}^{R}\right)\left(\underline{\eta}_{R} \otimes \operatorname{Id}_{S \otimes * S}\right)
\end{aligned}
$$




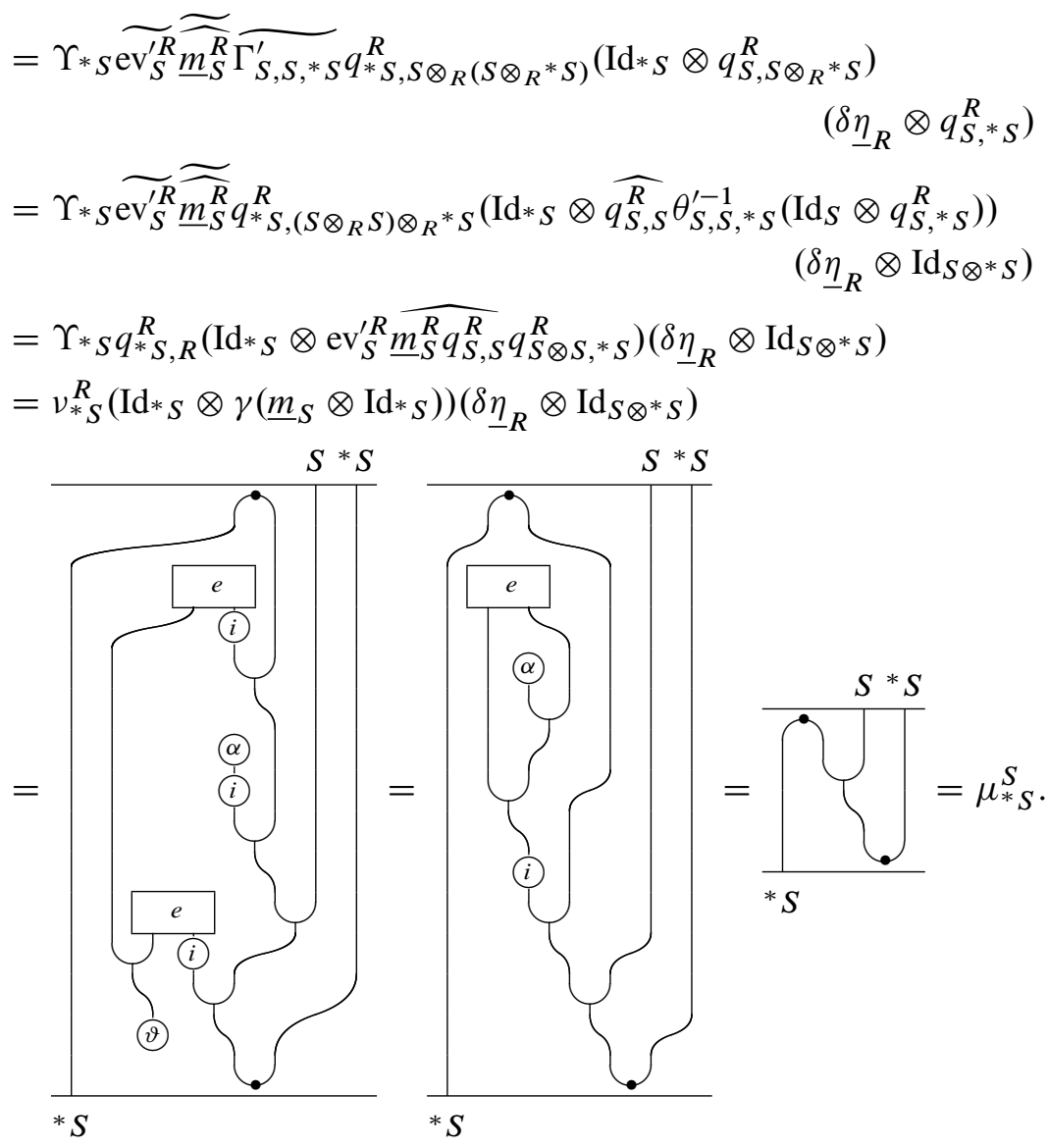

Using the above equality we have that $\Phi$ is left $S$-linear in ${ }_{R} \mathcal{C}_{R}$ if and only if

$$
\begin{aligned}
\mu_{* S}^{R, S} \widetilde{\Phi}=\Phi \underline{m}_{S}^{R} & \Leftrightarrow \mu_{* S}^{R, S} \widetilde{\Phi} q_{S, S}^{R}=\Phi \underline{m}_{S}^{R} q_{S, S}^{R} \\
& \Leftrightarrow \mu_{* S}^{R, S} q_{S,{ }^{*} S}^{R}\left(\operatorname{Id}_{S} \otimes \Phi\right)=\Phi \underline{m}_{S} \Leftrightarrow \mu_{* S}^{S}\left(\operatorname{Id}_{S} \otimes \Phi\right)=\Phi \underline{m}_{S},
\end{aligned}
$$

that is, if and only if $\Phi$ is left $S$-linear in $\mathcal{C}$. So our proof is finished.

We look now more carefully at the relation (7.1). In the case when $\mathcal{C}$ is a category of vector spaces it says that the restriction at $R$ of the Nakayama automorphism of $S$ is equal to the Nakayama automorphism of $R$. Our next aim is to provide a similar interpretation for (7.1) in a more general setting. Namely, the one provided by sovereign monoidal categories. Recall that a monoidal category is called sovereign if it is rigid and the left ()$^{*}$ and right ${ }^{*}()$ duality functors coincide.

The Nakayama automorphism of a Frobenius algebra in a sovereign monoidal category $\mathcal{C}$ was introduced in [20]. More exactly, if $A$ is a Frobenius algebra then by Theorem 5.1 we know that $A$ admits left and right dual objects. Since the category $\mathcal{C}$ 
is sovereign we have ${ }^{*} A=A^{*}:=\widehat{A}$, as objects in $\mathcal{C}$. Then the Nakayama automorphism of $A$ is defined as being
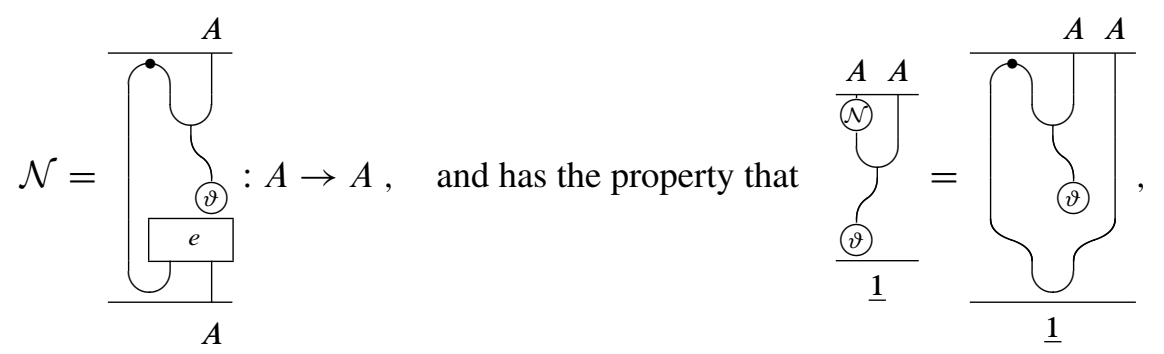

where $(\vartheta, e)$ is a Frobenius pair for $A . \mathcal{N}$ is a unital algebra isomorphism in $\mathcal{C}$, cf. [20, Proposition 18].

Theorem 7.4. Let $\mathcal{C}$ be a sovereign monoidal category, $R$ a Frobenius separable algebra and $i: R \rightarrow S$ an algebra extension in $\mathcal{C}$. Then $i: R \rightarrow S$ is Frobenius if and only if $S$ is Frobenius in $\mathcal{C}$ and $\widetilde{\mathcal{N}} \circ i=i \circ \mathcal{N}$, where $\widetilde{\mathcal{N}}$ and $\mathcal{N}$ are the Nakayama automorphisms of $S$ and $R$, respectively.

Proof. In view of Theorem 7.3 it is enough to prove that (7.1) is equivalent to $\widetilde{\mathcal{N}} \circ i=i \circ \mathcal{N}$. To this end denote by $(e, \vartheta)$ and $(\widetilde{e}, \widetilde{\vartheta})$ a Frobenius pair for $R$ and $S$, respectively. Since $\mathcal{C}$ is sovereign monoidal we have that ${ }^{*} \widetilde{e}=\widetilde{e}^{*}$, and this amounts to

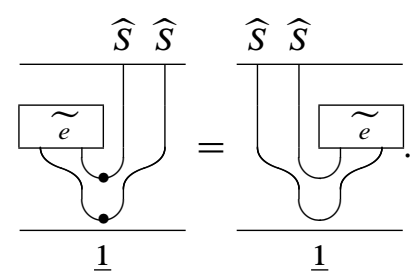

Also, in the proof of implication $(i) \Rightarrow$ (iii) in Theorem 5.1 the isomorphism $\Phi: S \rightarrow{ }^{*} S=\widehat{S}$ of left $S$-modules in $\mathcal{C}$ and its inverse $\phi$ are given by

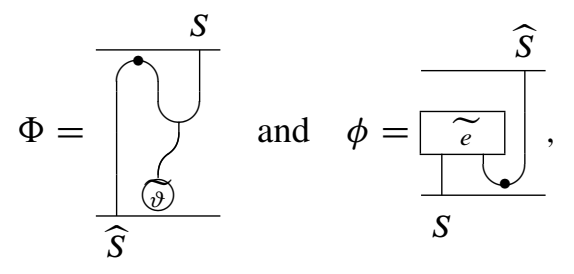

respectively. 
Thus we have that $\widetilde{\mathcal{N}} \circ i=i \circ \mathcal{N}$ is equivalent to
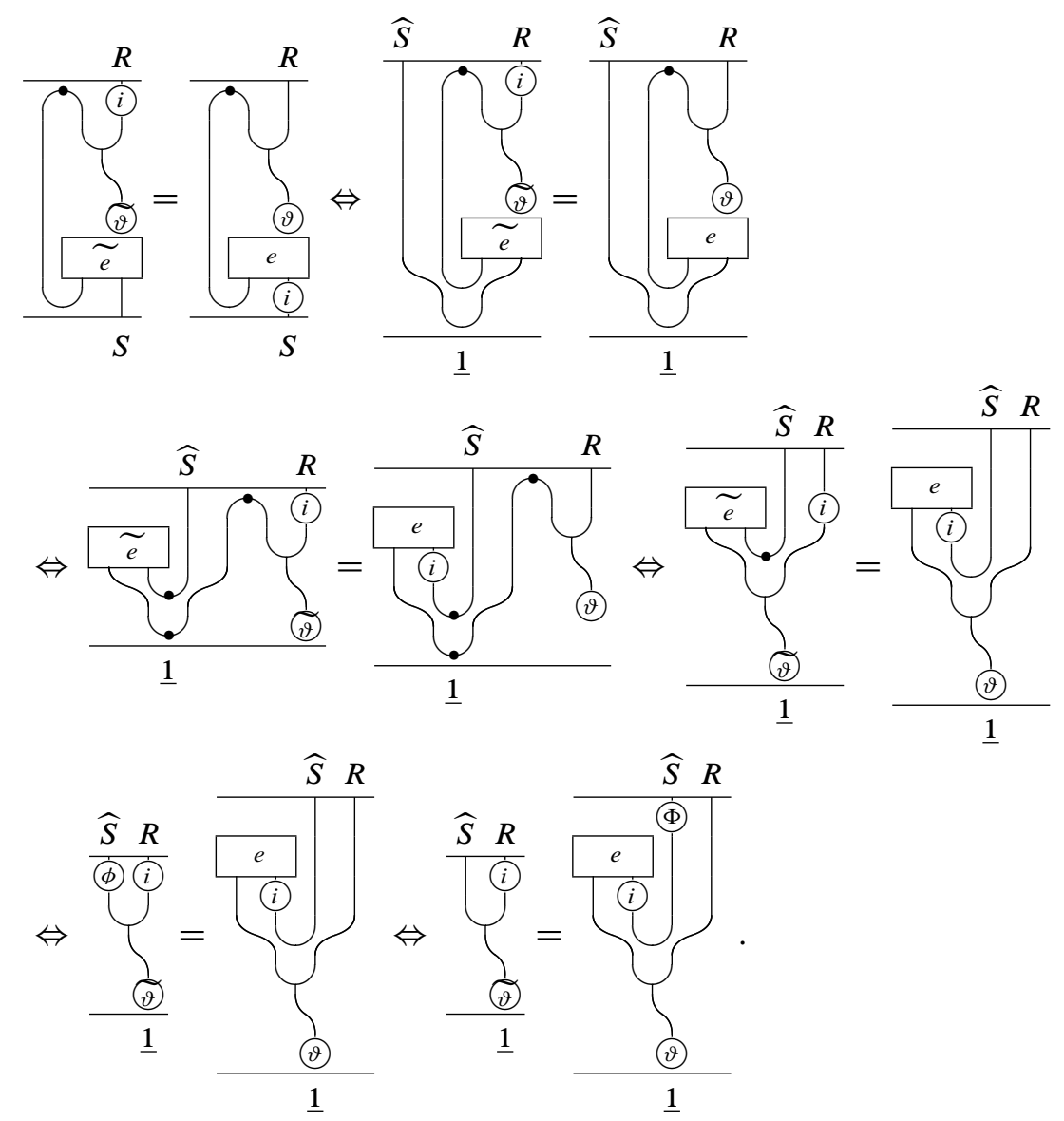

Finally, we compute that

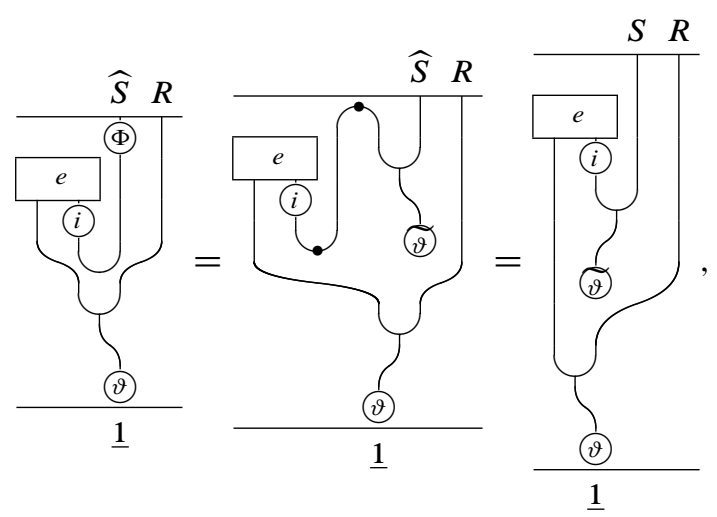

finishing the proof of the theorem. 


\section{Applications to wreaths}

As we already noticed, Theorem 5.1 can be also specialized either for a monad in an arbitrary category or for a monad in a 2-category. Actually the former is a particular case of the latter if we regard the monoidal category of endofunctors as an one object 2-category. This is why we restrict ourselves in presenting some characterizations for (separable) Frobenius monads in 2-categories only. These will be used later on for the characterization of a (separable) Frobenius wreath extension in a monoidal category.

Let $U$ be 0 -cell in a 2-category $\mathcal{K}$. Then $\mathcal{K}(U):=\mathcal{K}(U, U)$ is a monoidal category. The objects are 1-cells $U \rightarrow U$, morphisms are 2-cells, and the tensor product is given by horizontal composition of 2-cells. The unit is $1_{U}$, the unit 1cell on $U$. With this simple observation in mind it is immediate that a monad in a 2-category $\mathcal{K}$ is nothing but an algebra in a monoidal category $\mathcal{K}(A)$, for a certain 0 -cell $A$ of $\mathcal{K}$.

Recall that a monad $(A, t, \mu, \eta)$ in $\mathcal{K}$ is called Frobenius if there exist 2-cells $\vartheta: t \Rightarrow 1_{A}$ and $e: 1_{A} \Rightarrow t t$ such that the diagrams below are commutative<smiles>Oc1cccnc1</smiles>

A simple inspection shows that $(A, t, \mu, \eta)$ is Frobenius if and only if $(t, \mu, \eta)$ is a Frobenius algebra in the monoidal category $\mathcal{K}(A)$, so Theorem 5.1 applies. Note that the existence of a left (right) dual for an object $u: A \rightarrow A$ in the monoidal category $\mathcal{K}(A)$ reduces to the existence of a left (right) adjunction for $u$ in the 2categorical sense. Namely, $u$ has a left dual if there is a 1-cell $v: A \rightarrow A$ and 2-cells $\iota: 1_{A} \Rightarrow u v$ and $j: v u \Rightarrow 1_{A}$ such that $\left(1_{u} \odot j\right)\left(\iota \odot 1_{u}\right)=1_{u}$ and $\left(j \odot 1_{v}\right)\left(1_{v} \odot \iota\right)=1_{v}$. In this case we say also that $u$ is a right adjoint to $v$ and denote this adjunction as before, $(\iota, j): v \dashv u$.

In what follows the vertical composition in $\mathcal{K}$ will be denoted by juxtaposition.

Corollary 8.1. Let $\mathcal{K}$ be a 2 -category and $\mathbb{A}=(A, t, \mu, \eta)$ a monad in $\mathcal{K}$. Then the following assertions are equivalent:

(i) $\mathbb{A}$ is a Frobenius monad;

(ii) $(t, \mu, \eta)$ is a Frobenius algebra in the monoidal category $\mathcal{K}(A)$;

(iii) $t$ admits a coalgebra structure in the monoidal category $\mathcal{K}(A)$, say $(t, \delta: t \Rightarrow t t$, $\left.\varepsilon: t \Rightarrow 1_{A}\right)$, such that

$$
\left(1_{t} \odot \mu\right)\left(\delta \odot 1_{t}\right)=\delta \mu=\left(\mu \odot 1_{t}\right)\left(1_{t} \odot \delta\right) ;
$$

(iv) There exists an adjunction $(\rho, \lambda): t \dashv t$ such that $\lambda\left(\mu \odot 1_{t}\right)=\lambda\left(1_{t} \odot \mu\right)$; 
(v) There exists an adjunction of the form $(\rho, \vartheta \mu): t \dashv t$, with $\vartheta: t \Rightarrow 1_{A}$ a 2-cell in $\mathcal{K}$.

Proof. The equivalence between (i) and (ii) follows from the comments made above. The statements in (ii), (iii), (iv) and respectively (v) are precisely the ones in (i), (iv), (vii) and respectively (viii) in Theorem 5.1, specialized for the case when the monoidal category is $\mathcal{K}(A)$. We leave the details to the reader.

Of course we could add to Corollary 8.1 another four equivalent statements, namely the one corresponding to (ii), (iii), (v) and (vi) in Theorem 5.1, but for later use we prefer to keep only those that can be formulated in terms of the monad, and so as not to involve the existence of a dual object for the monad that is different from the monad itself. Also, remark that (ix) in Theorem 5.1 cannot be specialized for monads in 2-categories; it can be applied only in the situation when $1_{A}$, the unit object of $\mathcal{K}(A)$, is a left $\otimes$-generator for $\mathcal{K}(A)$. However, a more general treatment in this direction can be found in [29].

In the separable case we have a monoidal interpretation for the notion, too. First, a monad $(A, t, \mu, \eta)$ in a 2-category $\mathcal{K}$ is called separable if the multiplication $\mu$ splits as a $t$-bimodule, in the sense that there is a 2-cell $\gamma: t \Rightarrow t t$ in $\mathcal{K}$ such that the diagrams below are commutative

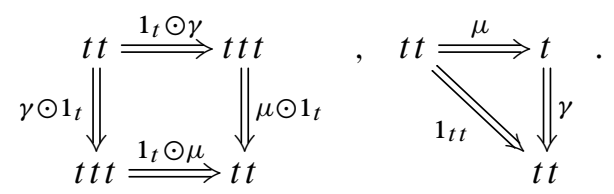

The proof of the next result is immediate, so we will omit it.

Proposition 8.2. For a monad $\mathbb{A}=(A, t, \mu, \eta)$ in a 2-category $\mathcal{K}$ the following assertions are equivalent:

(i) $\mathbb{A}$ is separable;

(ii) $(t, \mu, \eta)$ is a monoidal separable algebra in $\mathcal{K}(A)$;

(iii) There exists a 2-cell $e: 1_{A} \Rightarrow$ tt such that the diagrams below are commutative

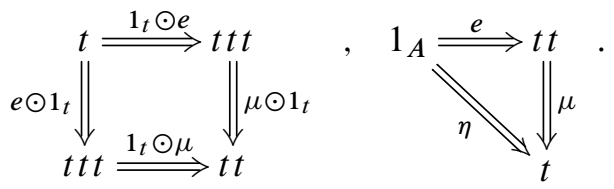

We move now to the Eilenberg-Moore 2-categories. Namely, to a 2-category $\mathcal{K}$ we can associate a new 2-category $E M(\mathcal{K})$, called the Eilenberg-Moore category associated to $\mathcal{K}$, cf. [27]. The 0 -cells in $E M(\mathcal{K})$ are monads in $\mathcal{K}, 1$-cells are the 
monad morphisms and 2-cells $(f, \psi) \stackrel{\rho}{\Longrightarrow}(g, \phi)$ are 2-cells $f \stackrel{\rho}{\Longrightarrow} g t$ in $\mathcal{K}$ obeying the equality

$$
\left(1_{g} \odot \mu_{t}\right)\left(\rho \odot 1_{t}\right) \psi=\left(1_{g} \odot \mu_{t}\right)\left(\phi \odot 1_{t}\right)\left(1_{s} \odot \rho\right) .
$$

The vertical composition of two 2-cells $(f, \psi) \stackrel{\rho}{\Longrightarrow}(g, \phi) \stackrel{\rho^{\prime}}{\Longrightarrow}(h, \gamma)$ is given by

$$
(f, \psi) \stackrel{\rho^{\prime} \circ \rho}{\Longrightarrow}(h, \gamma), \rho^{\prime} \circ \rho:=\left(1_{h} \odot \mu_{t}\right)\left(\rho^{\prime} \odot 1_{t}\right) \rho,
$$

while the horizontal composition of two cells

$$
\mathbb{A} \underset{\left(f^{\prime}, \psi^{\prime}\right)}{\stackrel{(f, \psi)}{\Downarrow \rho}} \mathbb{B} \frac{(g, \phi)}{\Downarrow \rho^{\prime}} \mathbb{C}
$$

is defined by $(g, \phi)(f, \psi)=\left(g f,\left(1_{g} \odot \psi\right) \circ\left(\phi \odot 1_{f}\right)\right)$, etc. and $g f \stackrel{\rho^{\prime} \oslash \rho}{\Longrightarrow} g^{\prime} f^{\prime} t$ given by

$$
\rho^{\prime} \oslash \rho:=\left(1_{g^{\prime}} \odot 1_{f^{\prime}} \odot \mu_{t}\right)\left(1_{g^{\prime}} \odot \rho \odot 1_{t}\right)\left(1_{g^{\prime}} \odot \psi\right)\left(\rho^{\prime} \odot 1_{f}\right) .
$$

The identity morphism of the 1 -cell $(f, \psi)$ is $1_{f} \odot \eta_{t}$, and for any monad $\mathbb{A}=$ $\left(A, t, \mu_{t}, \eta_{t}\right)$ in $\mathcal{K}$ we have $\left(1_{\mathbb{A}}, i_{\mathbb{A}}\right)=\left(\left(1_{A}, 1_{t}\right), \eta_{t}\right)$.

Motivated by the theory of entwined modules in $\mathcal{C}$-categories $[8,9]$, we are interested to study when the "algebra" extension produced by a monad in $E M(\mathcal{K})$ is Frobenius, respectively separable. Note that a (co)monad in $E M(\mathcal{K})$ is called a (co)wreath, so the main goal of this section is to see when an algebra extension defined by a wreath is Frobenius, and respectively separable. As we will see we can reduce this problem to the study of an algebra extension in a suitable monoidal category.

According to [27], a wreath is a monad $\mathbb{A}=(A, t, \mu, \eta)$ in $\mathcal{K}$ together with a 1-cell $A \stackrel{s}{\longrightarrow} A$ and 2-cells $t s \stackrel{\psi}{\Longrightarrow} s t, 1_{A} \stackrel{\sigma}{\Longrightarrow} s t$ and $s s \stackrel{\zeta}{\Longrightarrow} s t$ satisfying the following conditions:

$$
\begin{aligned}
& \left(1_{s} \odot \mu\right)\left(\psi \odot 1_{t}\right)\left(1_{t} \odot \psi\right)=\psi\left(\mu \odot 1_{s}\right), \psi\left(\eta \odot 1_{s}\right)=1_{s} \odot \eta ; \\
& \left(1_{s} \odot \mu\right)\left(\psi \odot 1_{t}\right)\left(1_{t} \odot \sigma\right)=\left(1_{s} \odot \mu\right)\left(\sigma \odot 1_{t}\right) ; \\
& \left(1_{s} \odot \mu\right)\left(\psi \odot 1_{t}\right)\left(1_{t} \odot \zeta\right)=\left(1_{s} \odot \mu\right)\left(\zeta \odot 1_{t}\right)\left(1_{s} \odot \psi\right)\left(\psi \odot 1_{s}\right) ; \\
& \left(1_{s} \odot \mu\right)\left(\zeta \odot 1_{t}\right)\left(1_{s} \odot \zeta\right)=\left(1_{s} \odot \mu\right)\left(\zeta \odot 1_{t}\right)\left(1_{s} \odot \psi\right)\left(\zeta \odot 1_{s}\right) ; \\
& \left(1_{s} \odot \mu\right)\left(\zeta \odot 1_{t}\right)\left(1_{s} \odot \sigma\right)=1_{s} \odot \eta ; \\
& \left(1_{s} \odot \mu\right)\left(\zeta \odot 1_{t}\right)\left(1_{s} \odot \psi\right)\left(\sigma \odot 1_{s}\right)=1_{s} \odot \eta .
\end{aligned}
$$

Lemma 8.3. Let $\mathcal{K}$ be a 2 -category. Then a wreath in $\mathcal{K}$ is nothing that an algebra in a monoidal category of the form $E M(\mathcal{K})(\mathbb{A})$, where $\mathbb{A}$ is a 0-cell in $E M(\mathcal{K})$, that is, a monad in $\mathcal{K}$. 
Proof. As we already have mentioned several times, since $E M(\mathcal{K})$ is a 2-category it follows that for any 0 -cell $\mathbb{A}=(A, t, \mu, \eta)$ of $E M(\mathcal{K})$ we have a monoidal structure on the category $\operatorname{EM}(\mathcal{K})(\mathbb{A})$. Furthermore, since a wreath is a monad in the 2category $\operatorname{EM}(\mathcal{K})$ it then follows that a wreath is an algebra in a monoidal category having the form mentioned in the statement.

For later use and also for the sake of the reader we next describe these structures explicitly. Namely,

- the objects of $\operatorname{EM}(\mathcal{K})(\mathbb{A})$ are the 1-cells of $\operatorname{EM}(\mathcal{K})$ from $\mathbb{A}$ to $\mathbb{A}$, that is, monad morphisms $(s, \psi)$ from $\mathbb{A}$ to $\mathbb{A}$;

- if $(s, \psi)$ and $\left(s^{\prime}, \psi^{\prime}\right)$ are monad morphisms from $\mathbb{A}$ to $\mathbb{A}$ then a morphism between $(s, \psi)$ and $\left(s^{\prime}, \psi^{\prime}\right)$ in $E M(\mathcal{K})(\mathbb{A})$ is a 2-cell $\rho:(s, \psi) \Rightarrow\left(s^{\prime}, \psi^{\prime}\right)$ in $E M(\mathcal{K})$;

- the composition of two morphisms in $E M(\mathcal{K})(\mathbb{A})$ is defined by the vertical composition of 2-cells in $E M(\mathcal{K})$ and the identity morphism corresponding to an object $(s, \psi)$ of $E M(\mathcal{K})(\mathbb{A})$ is $1_{s} \odot \eta$, where $\odot$ stands for the horizontal composition of 2-cells in $\mathcal{K}$;

- the monoidal structure is defined by the horizontal composition of 2-cells in $E M(\mathcal{K})$. More precisely, if $(s, \psi),\left(s^{\prime}, \psi^{\prime}\right)$ are objects of $E M(\mathcal{K})(\mathbb{A})$ we then define

$(s, \psi) \otimes\left(s^{\prime}, \psi^{\prime}\right)=\left(s^{\prime}, \psi^{\prime}\right)(s, \psi)=\left(s^{\prime} s,\left(\psi^{\prime} \odot 1_{s}\right)\left(1_{s^{\prime}} \odot \psi\right): s^{\prime} s t \Rightarrow t s^{\prime} s\right)$, and if $\rho:(s, \psi) \Rightarrow(f, \gamma)$ and $\rho^{\prime}:\left(s^{\prime}, \psi^{\prime}\right) \Rightarrow\left(f^{\prime}, \gamma^{\prime}\right)$ are two morphisms in $E M(\mathcal{K})(\mathbb{A})$ we then have $\rho \otimes \rho^{\prime}=\rho^{\prime} \oslash \rho$ as a 2-cell in $E M(\mathcal{K})$,

$$
\begin{aligned}
\rho \otimes \rho^{\prime}:(s, \psi) \otimes\left(s^{\prime}, \psi^{\prime}\right) & =\left(s^{\prime} s,\left(\psi^{\prime} \odot 1_{s}\right)\left(1_{s^{\prime}} \odot \psi\right)\right) \\
& \Rightarrow\left(f^{\prime} f,\left(\gamma^{\prime} \odot 1_{f}\right)\left(1_{f^{\prime}} \odot \gamma\right)\right)=(f, \gamma) \otimes\left(f^{\prime}, \gamma^{\prime}\right) ;
\end{aligned}
$$

- the unit object of $E M(\mathcal{K})(\mathbb{A})$ is $\left(1_{A}, 1_{t}\right)$ and for any object $(s, \psi)$ of $\operatorname{EM}(\mathcal{K})(\mathbb{A})$ we have $\operatorname{Id}_{(s, \psi)}=1_{s} \odot \eta$.

Now it can be easily verified that $(s, \psi): \mathbb{A} \rightarrow \mathbb{A}$ is an object of $E M(\mathcal{K})(\mathbb{A})$, i.e. a monad morphism, if and only if (8.1) holds. Then $(s, \psi)$ has an algebra structure in $E M(\mathcal{K})(\mathbb{A})$ if and only if there exist $\zeta:(s, \psi) \otimes(s, \psi) \rightarrow(s, \psi)$ and $\sigma:\left(1_{A}, 1_{t}\right) \rightarrow(s, \psi)$ morphisms in $E M(\mathcal{K})(\mathbb{A})$ such that $\zeta$ is associative and $\sigma$ is a unit for $\zeta$. The latter assertion can be restated in terms of the structure of $\mathcal{K}$ as follows:

- $\zeta$ and $\sigma$ are 2-cells in $\mathcal{K}, \zeta: s s \Rightarrow s t$ and $\sigma: 1_{A} \Rightarrow s t$, such that (8.3) and (8.2) hold;

- $\zeta$ is associative if and only if (8.4) is fulfilled;

- $\sigma$ is a unit for $\zeta$ if and only if (8.5) and (8.6) are satisfied.

Thus our proof is complete. 
Remark 8.4. Using arguments similar to the ones above we get that a cowreath in $\mathcal{K}$, that is a comonad in the 2-category $E M(\mathcal{K})$, is nothing but a coalgebra in a monoidal category of the form $\operatorname{EM}(\mathcal{K})(\mathbb{A})$, where $\mathbb{A}$ is a suitable monad in $\mathcal{K}$.

From now on we denote a wreath in $E M(\mathcal{K})$ by $(A, t, \mu, \eta, s, \psi, \zeta, \sigma)$ or, shortly, by $(\mathbb{A}, s, \psi, \zeta, \sigma)$ in the case when the structure of the monad $\mathbb{A}=(A, t, \mu, \eta)$ is fixed from the beginning. Following [27] to such a wreath we can associate the so-called wreath product. That is the monad in $\mathcal{K}$,

$$
\left(A, s t, \mu_{s t}: s t s t \stackrel{1_{s} \odot \psi \odot 1_{t}}{\Longrightarrow} s s t t \stackrel{1_{s} \odot 1_{s} \odot \mu}{\Longrightarrow} \text { sst } \stackrel{\zeta \odot 1_{t}}{\longrightarrow} \text { st } \stackrel{1_{s} \odot \mu}{\Longrightarrow} s t, \sigma: 1_{A} \Longrightarrow s t\right) \text {. }
$$

Otherwise stated, the wreath product is a monoidal algebra in $\mathcal{K}(A)$. The same is $(t, \mu, \eta)$ and we have $\iota:=\left(1_{s} \odot \mu\right)\left(\sigma \odot 1_{t}\right): t \Rightarrow s t$ an algebra morphism in $\mathcal{K}(A)$ since

$$
\begin{aligned}
\mu_{s t}(\iota \odot \iota) & =\left(1_{s} \odot \mu\right)\left(\zeta \odot 1_{t}\right)\left(1_{s} \odot 1_{s} \odot \mu\right)\left(1_{s} \odot \mu \odot 1_{t}\right)\left(1_{s} \odot 1_{t} \odot \iota\right)\left(\iota \odot 1_{t}\right) \\
& =\left(1_{s} \odot \mu\right)\left(\zeta \odot 1_{t}\right)\left(1_{s} \odot\left(1_{s} \odot \mu\left(\mu \odot 1_{t}\right)\right)\left(\psi \odot 1_{t} \odot 1_{t}\right)\right. \\
& \left.\left(1_{s} \odot \sigma \odot 1_{t}\right)\right)\left(\iota \odot 1_{t}\right) \\
& \stackrel{\left(8^{2.2}\right)}{=}\left(1_{s} \odot \mu\right)\left(\zeta \odot 1_{t}\right)\left(1_{s} \odot\left(1_{s} \odot \mu\right)\left(\left(1_{s} \odot \mu\right)\left(\sigma \odot 1_{t}\right) \odot 1_{t}\right)\right)\left(\iota \odot 1_{t}\right) \\
& =\left(1_{s} \odot \mu\right)\left(\zeta \odot 1_{t}\right)\left(1_{s} \odot\left(1_{s} \odot \mu\right)\left(\sigma \odot 1_{t}\right) \mu\right)\left(\iota \odot 1_{t}\right) \\
& =\left(1_{s} \odot \mu\right)\left(1_{s} \odot 1_{t} \odot \mu\right)\left(\zeta \odot 1_{t} \odot 1_{t}\right)\left(1_{s} \odot\left(\sigma \odot 1_{t}\right) \mu\right)\left(\iota \odot 1_{t}\right) \\
& =\left(1_{s} \odot \mu\right)\left(\left(1_{s} \odot \mu\right)\left(\zeta \odot 1_{t}\right)\left(1_{s} \odot \sigma\right) \odot 1_{t}\right)\left(1_{s} \odot \mu\right)\left(\iota \odot 1_{t}\right) \\
& \stackrel{(8.5)}{=}\left(1_{s} \odot \mu\right)\left(\iota \odot 1_{t}\right) \\
& =\left(1_{s} \odot \mu\right)\left(1_{s} \odot 1_{t} \odot \mu\right)\left(\sigma \odot 1_{t} \odot 1_{t}\right)=\left(1_{s} \odot \mu\right)\left(\sigma \odot 1_{t}\right) \mu=\iota \mu
\end{aligned}
$$

and $\iota \eta=\left(1_{s} \odot \mu\right)\left(\sigma \odot 1_{t}\right) \eta=\left(1_{s} \odot \mu\left(1_{t} \odot \eta\right)\right) \sigma=\sigma$.

Definition 8.5. The canonical monad extension associated to a wreath $(\mathbb{A}, s, \psi, \zeta, \sigma)$ in $\mathcal{K}$ is the monad morphism $\left(1_{A}, \iota\right): \mathbb{A} \rightarrow\left(A, s t, \mu_{s t}, \sigma\right)$ in $\mathcal{K}$. We call this canonical monad extension Frobenius, respectively separable, if $\mathcal{K}(A)$ admits coequalizers and any object of it is coflat, and, moreover, the associated algebra extension $\iota: t \Rightarrow s t$ is Frobenius, respectively separable, in the monoidal category $\mathcal{K}(A)$.

Due to the monoidal flavor of the above definition we have the following characterizations for the canonical monad extension associated to a wreath to be Frobenius.

Theorem 8.6. Let $(A, t, \mu, \eta, s, \psi, \zeta, \sigma)$ be a wreath in $\mathcal{K}$. Then the following assertions are equivalent:

(i) $(A, t, \mu, \eta, s, \psi, \zeta, \sigma)$ is a Frobenius monad in $E M(\mathcal{K})$, that is a Frobenius wreath in $\mathcal{K}$;

(ii) $(s, \psi)$ is a Frobenius algebra in the monoidal category $\operatorname{EM}(\mathcal{K})(\mathbb{A})$; 
(iii) $(s, \psi)$ admits a coalgebra structure in $E M(\mathcal{K})(\mathbb{A})$ with the comultiplication structure morphism $(s, \psi)$-bilinear, that is there exists a cowreath structure in $\mathcal{K}$ of the form

$$
(\mathbb{A}, s, \psi, s \stackrel{\delta}{\Longrightarrow} s s t, s \stackrel{\varepsilon}{\Longrightarrow} t)
$$

such that

$$
\begin{aligned}
\left(1_{s} \odot 1_{s} \odot \mu\right)\left(\delta \odot 1_{t}\right) \zeta & =\left(1 s \odot 1_{s} \odot \mu\right)\left(1_{s} \odot \zeta \odot 1_{t}\right)\left(1_{s} \odot 1_{s} \odot \psi\right)\left(\delta \odot 1_{s}\right) \\
& =\left(1_{s} \odot 1_{s} \odot \mu\right)\left(1_{s} \odot \psi \odot 1_{t}\right)\left(\zeta \odot 1_{s} \odot 1_{t}\right)\left(1_{s} \odot \delta\right) ;
\end{aligned}
$$

(iv) There exists an adjunction $(\rho, \lambda):(s, \psi) \dashv(s, \psi)$ in $E M(\mathcal{K})(\mathbb{A})$ such that $\lambda$ is associative, this means,

$$
\mu\left(\lambda \odot 1_{t}\right)\left(1_{s} \odot \psi\right)\left(\zeta \odot 1_{s}\right)=\mu\left(\lambda \odot 1_{t}\right)\left(1_{s} \odot \zeta\right) ;
$$

(v) There is an adjunction $(\rho, \lambda):(s, \psi) \dashv(s, \psi)$ in $E M(\mathcal{K})(\mathbb{A})$ with $\lambda$ having the form $\lambda=\mu\left(\vartheta \odot 1_{t}\right) \zeta: s s \Rightarrow t$, for some 2-cell $\vartheta: s \Rightarrow t$ in $\mathcal{K}$.

If $\mathcal{K}(A)$ admits coequalizers and any object of it is coflat then $(i)-(v)$ above are also equivalent to

(vi) The canonical monad extension associated to $(A, t, \mu, \eta, s, \psi, \zeta, \sigma)$ is Frobenius.

Proof. The equivalences between (ii), (iii), (iv), (v) and (vi) follow from Corollary 8.1 applied to the monad $(A, t, \mu, \eta, s, \psi, \zeta, \sigma)$ in the 2-category $E M(\mathcal{K})$. The difficult part is to prove the equivalence between (i) and (vi), provided that $\mathcal{K}(A)$ admits coequalizers and any object of it is coflat.

$(i) \Leftrightarrow(v i)$. We have that $\iota: t \Rightarrow s t$ is a Frobenius extension if and only if there exist 2-cells $\vartheta: s t \Rightarrow t$ and $e: 1_{A} \Rightarrow(s t) \otimes_{t}(s t)$ in $\mathcal{K}$ such that $\vartheta$ is $t$-bilinear and the diagrams in Theorem 3.5 (ii), specialized for the context provided by the category $\mathcal{C}=\mathcal{K}(A)$, are commutative.

It is obvious to check that giving a $t$-bilinear morphism $\vartheta: s t \Rightarrow t$ in $\mathcal{K}(A)$ is equivalent to giving a morphism $\varsigma: s \Rightarrow t$ in $\mathcal{K}$ such that

$$
\mu\left(\varsigma \odot 1_{t}\right) \psi=\mu\left(1_{t} \odot \varsigma\right): t s \Rightarrow t .
$$

Indeed to $\vartheta$ corresponds $\varsigma=\vartheta\left(1_{s} \odot \eta\right)$, while $\vartheta$ can be recovered from $\varsigma$ as $\vartheta=\mu\left(\varsigma \odot 1_{t}\right)$. Note that (8.7) is imposed by the right $t$-linearity of $\vartheta$.

Similarly, thinking in the monoidal sense, we have

$$
(s t) \otimes_{t}(s t)=(t \otimes s) \otimes_{t}(t \otimes s) \stackrel{\Upsilon_{t \otimes s, s}}{\longrightarrow} t \otimes s \otimes s=s s t,
$$

and so to give $e$ is equivalent to give a 2 -cell $\kappa: 1_{A} \Rightarrow s s t$ in $\mathcal{K}$. Now, as in the previous proofs involving coequalizers, one can show that the pair $(\vartheta, e)$ is a 
Frobenius pair for the extension $\iota: t \Rightarrow s t$ if and only if $\varsigma$ satisfies (8.7) and the following equalities hold,

$$
\begin{array}{r}
\left(1_{s s} \odot \mu\right)\left(1_{s} \odot \psi \odot 1_{t}\right)\left(1_{s} \odot \mu \odot 1_{s t}\right)\left(\zeta \odot 1_{t s t}\right)\left(1_{s} \odot \psi \odot 1_{s t}\right)\left(1_{s t} \odot \kappa\right) \\
=\left(1_{s s} \odot \mu\right)\left(1_{s} \odot \zeta \odot 1_{t}\right)\left(1_{s s s} \odot \mu\right)\left(1_{s s} \odot \psi \odot 1_{t}\right)(\kappa \odot 1 s t) ; \\
\left.\sigma=\left(1_{s} \odot \mu\right)\left(1_{s} \odot \varsigma \odot 1_{t}\right) \kappa=\left(1_{s} \odot \mu\right)\left(\psi \odot 1_{t}\right)\right)\left(\varsigma \odot 1_{s t}\right) \kappa .
\end{array}
$$

We claim now that (8.8) is equivalent to the following two equalities:

$$
\left(1_{s s} \odot \mu\right)\left(\kappa \odot 1_{t}\right)=\left(1_{s s} \odot \mu\right)\left(1_{s} \odot \psi \odot 1_{t}\right)\left(\psi \odot 1_{s t}\right)\left(1_{t} \odot \kappa\right)
$$

and

$$
\begin{aligned}
\left(1_{s s} \odot \mu\right)\left(1_{s} \odot \psi \odot 1_{t}\right) & \left(\zeta \odot 1_{s t}\right)\left(1_{s} \odot \kappa\right) \\
& =\left(1_{s s} \odot \mu\right)\left(1_{s} \odot \zeta \odot 1_{t}\right)\left(1_{s s} \odot \psi\right)\left(\kappa \odot 1_{s}\right) .
\end{aligned}
$$

This would have as a consequence the following equivalence: $(\vartheta, e)$ is a Frobenius pair for $\iota: t \Rightarrow s t$ if and only of there exists a pair $\left(s: s \Rightarrow t, \kappa: 1_{A} \Rightarrow s s t\right)$ such that (8.7), (8.10), (8.11) and (8.9) hold. But these relations have the following meaning:

- (8.7) says that $\varsigma:(s, \psi) \Rightarrow\left(1_{A}, 1_{t}\right)$ is a morphism in $\operatorname{EM}(\mathcal{K})(\mathbb{A})$;

- (8.10) says that $\kappa:\left(1_{A}, 1_{t}\right) \Rightarrow(s, \psi) \otimes(s, \psi)=\left(s s,\left(\psi \odot 1_{s}\right)\left(1_{s} \odot \psi\right)\right)$ is a morphism in $E M(\mathcal{K})(\mathbb{A})$ as well;

- (8.11) expresses the commutativity of the diagram

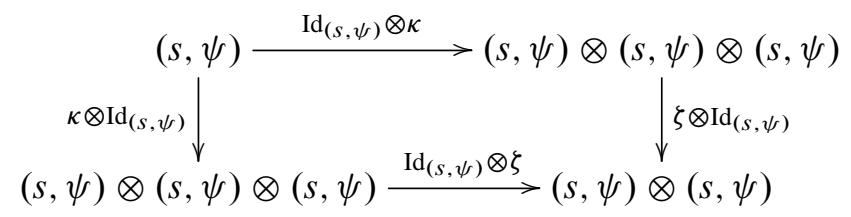

in $\operatorname{EM}(\mathcal{K})(\mathbb{A})$, and

- (8.9) can be restated in terms of the monoidal structure of $\operatorname{EM}(\mathcal{K})(\mathbb{A})$ as

$$
\begin{aligned}
\left(\left(1_{A}, 1_{t}\right) \stackrel{e}{\longrightarrow}(s, \psi)\right. & \left.\otimes(s, \psi) \stackrel{s \otimes \operatorname{Id}_{(s, \psi)}}{\longrightarrow}(s, \psi)\right)=\sigma \\
& =\left(\left(1_{A}, 1_{t}\right) \stackrel{e}{\longrightarrow}(s, \psi) \otimes(s, \psi) \stackrel{\operatorname{Id}_{(s, \psi)} \otimes S}{\longrightarrow}(s, \psi)\right) .
\end{aligned}
$$

Thus $(\vartheta, e)$ is a Frobenius pair for $i: t \Rightarrow s t$ in $\mathcal{K}(A)$ if and only if $(\varsigma, \kappa)$ is a Frobenius pair for $(s, \psi)$ in $E M(\mathcal{K})(\mathbb{A})$, as desired. 
So it remains to show that (8.8) is equivalent to (8.10) and (8.11). Towards this end, we first show that by composing both sides of (8.8) to the right with $\left(1_{s} \odot \mu\right)$ $\left(\sigma \odot 1_{t}\right)$ we get (8.10). Indeed, on one hand we have

$$
\begin{gathered}
\left(1_{s s} \odot \mu\right)\left(1_{s} \odot \psi \odot 1_{t}\right)\left(1_{s} \odot \mu \odot 1_{s t}\right)\left(\zeta \odot 1_{t s t}\right)\left(1_{s} \odot \psi \odot 1_{s t}\right)\left(1_{s t} \odot \kappa\right) \\
\left(1_{s} \odot \mu\right)\left(\sigma \odot 1_{t}\right) \\
=\left(1_{s s} \odot \mu\right)\left(1_{s} \odot \psi \odot 1_{t}\right)\left(1_{s} \odot \mu \odot 1_{s t}\right)\left(\zeta \odot 1_{t s t}\right)\left(1_{s} \odot \psi\left(\mu \odot 1_{s}\right) \odot 1_{s t}\right) \\
\left(1_{s t t} \odot \kappa\right)\left(\sigma \odot 1_{t}\right) \\
\stackrel{(8.1)}{=}\left(1_{s s} \odot \mu\right)\left(1_{s} \odot \psi \odot 1_{t}\right)\left(1_{s} \odot \mu\left(1_{t} \odot \mu\right) \odot 1_{s t}\right)\left(\zeta \odot 1_{t t s t}\right) \\
\left(1_{s} \odot\left(\psi \odot 1_{t}\right)\left(1_{t} \odot \psi\right) \odot 1_{s t}\right)\left(\sigma \odot 1_{s t s t}\right)\left(1_{t} \odot \kappa\right) \\
=\left(1_{s s} \odot \mu\right)\left(1_{s} \odot \psi \odot 1_{t}\right)\left(1_{s} \odot \mu \odot 1_{s t}\right) \\
\left(\left(1_{s} \odot \mu\right)\left(\zeta \odot 1_{t}\right)\left(1_{s} \odot \psi\right)\left(\sigma \odot 1_{s}\right) \odot 1_{t s t}\right)\left(\psi \odot 1_{s t}\right)\left(1_{t} \odot \kappa\right) \\
\stackrel{(8.6)}{=}\left(1_{s s} \odot \mu\right)\left(1_{s} \odot \psi \odot 1_{t}\right)\left(\psi \odot 1_{s t}\right)\left(1_{t} \odot \kappa\right) .
\end{gathered}
$$

On the other hand, using (8.2) and (8.5) we deduce that

$$
\begin{array}{r}
\left(1_{s s} \odot \mu\right)\left(1_{s} \odot \zeta \odot 1_{t}\right)\left(1_{s s s} \odot \mu\right)\left(1_{s s} \odot \psi \odot 1_{t}\right)(\kappa \odot 1 s t)\left(1_{s} \odot \mu\right)\left(\sigma \odot 1_{t}\right) \\
=\left(1_{s s} \odot \mu\right)\left(\kappa \odot 1_{t}\right),
\end{array}
$$

as required. In a similar manner, if we compose both sides of (8.8) to the right with $1_{s} \odot \eta$ we obtain (8.11), and this is essentially due to the second equality in (8.1).

For the converse, (8.10) and (8.11) imply (8.8) since

$$
\begin{aligned}
&\left(1_{s s} \odot \mu\right)\left(1_{s} \odot \zeta \odot 1_{t}\right)\left(1_{s s s} \odot \mu\right)\left(1_{s s} \odot \psi \odot 1_{t}\right)\left(\kappa \odot 1_{s t}\right) \\
&=\left(1_{s s} \odot \mu\right)\left(\left(1_{s s} \odot \mu\right)\left(1_{s} \odot \zeta \odot 1_{t}\right)\left(1_{s s} \odot \psi\right)\left(\kappa \odot 1_{s}\right) \odot 1_{t}\right) \\
& \stackrel{(8.11)}{=}\left(1_{s s} \odot \mu\right)\left(1_{s s t} \odot \mu\right)\left(1_{s} \odot \psi \odot 1_{t t}\right)\left(\zeta \odot 1_{s t t}\right)\left(1_{s} \odot \kappa \odot 1_{t}\right) \\
&=\left(1_{s s} \odot \mu\right)\left(1_{s} \odot \psi \odot 1_{t}\right)\left(\zeta \odot 1_{s t}\right)\left(1_{s} \odot\left(1_{s s} \odot \mu\right)\left(\kappa \odot 1_{t}\right)\right) \\
& \stackrel{(8.10)}{=}\left(1_{s s} \odot \mu\right)\left(1_{s s} \odot \mu \odot 1_{t}\right)\left(1_{s} \odot \psi \odot 1_{t t}\right)\left(\zeta \odot 1_{s t t}\right)\left(1_{s s} \odot \psi \odot 1_{t}\right)\left(1_{s} \odot \psi \odot 1_{s t}\right)\left(1_{s t} \odot \kappa\right) \\
&=\left(1_{s s} \odot \mu\right)\left(1_{s} \odot\left(1_{s} \odot \mu\right)\left(\psi \odot 1_{t}\right)\left(1_{t} \odot \psi\right) \odot 1_{t}\right)\left(\zeta \odot 1_{t s t}\right) \\
&\left(1_{s} \odot \psi \odot 1_{s t}\right)\left(1_{s t} \odot \kappa\right) \\
& \stackrel{(8.1)}{=}\left(1_{s s} \odot \mu\right)\left(1_{s} \odot \psi \odot 1_{t}\right)\left(1_{s} \odot \mu \odot 1_{s t}\right)\left(\zeta \odot 1_{t s t}\right)\left(1_{s} \odot \psi \odot 1_{s t}\right)
\end{aligned}
$$

as desired. This finishes the proof of the theorem.

We focus now on the separability case. Similar to Theorem 8.6 we have the following characterizations for the canonical monad extension associated to a wreath to be separable. 
Theorem 8.7. Let $(A, t, \mu, \eta, s, \psi, \zeta, \sigma)$ be a wreath in $\mathcal{K}$. Then the following assertions are equivalent:

(i) $(A, t, \mu, \eta, s, \psi, \zeta, \sigma)$ is a separable monad in $E M(\mathcal{K})$, that is a separable wreath in $\mathcal{K}$;

(ii) $(s, \psi)$ is a separable algebra in the monoidal category $\operatorname{EM}(\mathcal{K})(\mathbb{A})$.

If $\mathcal{K}(A)$ admits coequalizers and any object of it is coflat then (i)-(ii) above are also equivalent to

(iii) The canonical monad extension associated to $(A, t, \mu, \eta, s, \psi, \zeta, \sigma)$ is separable.

Proof. The wreath $(A, t, \mu, \eta, s, \psi, \zeta, \sigma)$ is actually a monad $(s, \psi)$ in $\operatorname{EM}(\mathcal{K})((A, t$, $\mu, \eta)$ ) and moreover, it is separable if and only if $(s, \psi)$ is so within the monoidal category $\operatorname{EM}(\mathcal{K})((A, t, \mu, \eta))$. This shows the equivalences between (i) and (ii). Furthermore, one can see easily that both are equivalent to the fact that there exists a 2-cell $\left(1_{A}, 1_{t}\right) \stackrel{e}{\Longrightarrow}\left(s s,\left(1_{s} \odot \psi\right)\left(\psi \odot 1_{s}\right)\right)$ in $\operatorname{EM}(\mathcal{K})(\mathbb{A})$, that is, a 2-cell $e: 1_{A} \Rightarrow$ sst in $\mathcal{K}$ for which the diagram

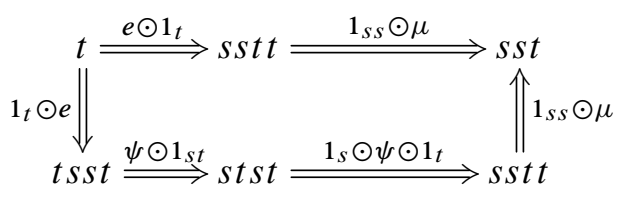

is commutative, such that the following equalities hold:

$$
\begin{aligned}
\left(1_{s} \odot\left(1_{s} \odot \mu\right)\left(\zeta \odot 1_{t}\right)\right. & \left.\left(1_{s} \odot \psi\right)\right)\left(e \odot 1_{s}\right) \\
& =\left(1_{s} \odot\left(1_{s} \odot \mu\right)\left(\psi \odot 1_{t}\right)\right)\left(\zeta \odot 1_{s t}\right)\left(1_{s} \odot e\right), \\
\left(1_{s} \odot \mu\right)\left(\zeta \odot 1_{t}\right) e & =\sigma .
\end{aligned}
$$

We prove now the equivalence between (i) and (iii), so we are under the hypothesis that $\operatorname{EM}(\mathcal{K})(\mathbb{A})$ admits coequalizers and every object of it is coflat. Then the extension $\iota: t \Rightarrow s t$ is separable in $\mathcal{K}(A)$ if and only if there exists a 2-cell $e: 1_{A}: \Rightarrow(s t) \otimes_{t}(s t)$ in $\mathcal{K}$ that belongs to $\mathcal{W}$ and obeys $\zeta^{t} e=\sigma$, where $\mathcal{W}$ is the set defined in Theorem 3.6, specialized for the category $\mathcal{K}(A)$. As in the proof of Theorem 8.6 one can easily verify that this is equivalent to the existence of a 2-cell $e: 1_{A} \Rightarrow$ sst in $\mathcal{K}$ such that (8.14) is satisfied and

$$
\begin{aligned}
& \left(1_{s} \odot\left(1_{s} \odot \mu\right)(\zeta \odot \mu)\left(1_{s} \odot \psi \odot 1_{t}\right)\right)\left(e \odot 1_{s t}\right) \\
= & \left(1_{s} \odot\left(1_{s} \odot \mu\right)\left(\psi \odot 1_{t}\right)\right)\left(\zeta \odot 1_{s} \odot \mu\right)\left(1_{s} \odot\left(1_{s} \odot \psi\right)\left(\psi \odot 1_{s}\right) \odot 1_{t}\right)\left(1_{s t} \odot e\right) .
\end{aligned}
$$


Actually, by (8.1) it follows that (8.15) is equivalent to

$\left(1_{s} \odot\left(1_{s} \odot \mu\right)(\zeta \odot \mu)\left(1_{s} \odot \psi \odot 1_{t}\right)\right)\left(e \odot 1_{s t}\right)$

$=\left(1_{s} \odot\left(1_{s} \odot \mu\right)\left(\psi \odot 1_{t}\right)\left(\mu \odot 1_{s t}\right)\right)\left(\left(\zeta \odot 1_{t}\right)\left(1_{s} \odot \psi\right) \odot 1_{s t}\right)\left(1_{s t} \odot e\right)$.

We state that (8.16) is equivalent to (8.12) and (8.13), and this will end the proof. Indeed, if we compose both sides of (8.16) to the right with $1_{s} \odot \eta$ we then get (8.13). To get (8.12) we compose both sides of (8.16) to the right with $\iota=\left(1_{s} \odot \mu\right)$ $\left(\psi \odot 1_{t}\right)\left(1_{t} \odot \sigma\right): t \Rightarrow s t$. On one hand we have

$$
\begin{gathered}
\left(1_{s} \odot\left(1_{s} \odot \mu\right)\left(\psi \odot 1_{t}\right)\left(\mu \odot 1_{s t}\right)\right)\left(\left(\zeta \odot 1_{t}\right)\left(1_{s} \odot \psi\right) \odot 1_{s t}\right)\left(1_{s t} \odot e\right) \\
\left(1_{s} \odot \mu\right)\left(\psi \odot 1_{t}\right)\left(1_{t} \odot \sigma\right) \\
=\left(1_{s} \odot\left(1_{s} \odot \mu\right)\left(\psi \odot 1_{t}\right)\left(\mu \odot 1_{s t}\right)\right)\left(\zeta \odot 1_{t s t}\right)\left(1_{s} \odot \psi\left(\mu \odot 1_{s}\right) \odot 1_{s t}\right) \\
\left(\psi \odot{ }_{t s t}\right)\left(1_{t s t} \odot e\right)\left(1_{t} \odot \sigma\right) \\
\stackrel{(8.1)}{=}\left(1_{s} \odot\left(1_{s} \odot \mu\right)\left(\psi \odot 1_{t}\right)\left(\mu \odot 1_{s t}\right)\left(1_{t} \odot \mu \odot 1_{s t}\right)\right) \\
\left(\left(\zeta \odot 1_{t t}\right)\left(1_{s} \odot \psi \odot 1_{t}\right)\left(1_{s t} \odot \psi\right)\left(\psi \odot 1_{t s}\right) \odot 1_{s t}\right)\left(1_{t s t} \odot e\right)\left(1_{t} \odot \sigma\right) \\
=\left(1_{s} \odot\left(1_{s} \odot \mu\right)\left(\psi \odot 1_{t}\right)\left(\mu \odot 1_{s t}\right)\right) \\
\left(\left(1_{s} \odot \mu\right)\left(\zeta \odot 1_{t}\right)\left(1_{s} \odot \psi\right)\left(\psi \odot 1_{s}\right) \odot 1_{t s t}\right)\left(1_{t s} \odot \psi \odot 1_{s t}\right)\left(1_{t s t} \odot e\right)\left(1_{t} \odot \sigma\right) \\
\stackrel{(8.3)}{=}\left(1_{s} \odot\left(1_{s} \odot \mu\right)\left(\psi \odot 1_{t}\right)\left(\mu \odot 1_{s t}\right)\left(1_{t} \odot \mu \odot 1_{s t}\right)\right) \\
\quad\left(\left(\psi \odot 1_{t}\right)\left(1_{t} \odot \zeta\right) \odot 1_{t s t}\right)\left(1_{t s} \odot \psi \odot 1_{s t}\right)\left(1_{t} \odot \sigma \odot 1_{s s t}\right)\left(1_{t} \odot e\right) \\
=\left(1_{s} \odot\left(1_{s} \odot \mu\right)\left(\psi \odot 1_{t}\right)\left(\mu \odot 1_{s t}\right)\right)\left(\psi \odot 1_{t s t}\right) \\
\left(1_{t} \odot\left(1_{s} \odot \mu\right)\left(\zeta \odot 1_{t}\right)\left(1_{s} \odot \psi\right)\left(\sigma \odot 1_{s}\right) \odot 1_{s t}\right)\left(1_{t} \odot e\right) \\
\stackrel{(8.6)}{=}\left(1_{s} \odot\left(1_{s} \odot \mu\right)\left(\psi \odot 1_{t}\right)\right)\left(\psi \odot 1_{s t}\right)\left(1_{t} \odot e\right) .
\end{gathered}
$$

On the other hand,

$$
\begin{aligned}
\left(1_{s} \odot\right. & \left.\left(1_{s} \odot \mu\right)(\zeta \odot \mu)\left(1_{s} \odot \psi \odot 1_{t}\right)\right)\left(e \odot 1_{s t}\right)\left(1_{s} \odot \mu\right)\left(\psi \odot 1_{t}\right)\left(1_{t} \odot \sigma\right) \\
= & \left(1_{s} \odot\left(1_{s} \odot \mu\right)(\zeta \odot \mu)\right)\left(1_{s s} \odot\left(\psi \odot 1_{t}\right)\left(1_{t s} \odot \mu\right)\right) \\
& \left(1_{s s t} \odot\left(\psi \odot 1_{t}\right)\left(1_{t} \odot \sigma\right)\right)\left(e \odot 1_{t}\right) \\
= & \left(1_{s} \odot\left(1_{s} \odot \mu\right)\left(\zeta \odot 1_{t}\right)\right)\left(1_{s s s} \odot \mu\right)\left(1_{s s} \odot\left(1_{s t} \odot \mu\right)\left(\psi \odot 1_{t t}\right)\right) \\
& \left(1_{s s t} \odot\left(\psi \odot 1_{t}\right)\left(1_{t} \odot \sigma\right)\right)\left(e \odot 1_{t}\right) \\
& \stackrel{(8.1)}{=}\left(1_{s} \odot\left(1_{s} \odot \mu\right)\left(\zeta \odot 1_{t}\right)\right)\left(1_{s s} \odot\left(1_{s} \odot \mu\right)\left(\psi \odot 1_{t}\right)\left(1_{t} \odot \sigma\right)\right) \\
& \stackrel{\left(1_{s s} \odot \mu\right)\left(e \odot 1_{t}\right)}{=}\left(1_{s s} \odot \mu\left(1_{t} \odot \mu\right)\right)\left(1_{s} \odot \zeta \odot 1_{t t}\right)\left(1_{s s} \odot\left(\sigma \odot 1_{t}\right) \mu\right)\left(e \odot 1_{t}\right) \\
= & \left(1_{s s} \odot \mu\right)\left(1_{s} \odot\left(1_{s} \odot \mu\right)\left(\zeta \odot 1_{t}\right)\left(1_{s} \odot \sigma\right) \odot 1_{t}\right)\left(1_{s s} \odot \mu\right)\left(e \odot 1_{t}\right) \\
& \stackrel{(8.5)}{=}\left(1_{s s} \odot \mu\right)\left(e \odot 1_{t}\right) .
\end{aligned}
$$


Comparing the results of the above two computations we deduce (8.13), as stated. The converse is also true since

$$
\begin{gathered}
\left(1_{s} \odot\left(1_{s} \odot \mu\right)(\zeta \odot \mu)\left(1_{s} \odot \psi \odot 1_{t}\right)\right)\left(e \odot 1_{s t}\right) \\
=\left(1_{s s} \odot \mu\right)\left(1_{s} \odot\left(1_{s} \odot \mu\right)\left(\zeta \odot 1_{t}\right)\left(1_{s} \odot \psi\right) \odot 1_{t}\right)\left(e \odot 1_{s t}\right) \\
\stackrel{(8.13)}{=}\left(1_{s s} \odot \mu\left(\mu \odot 1_{t}\right)\right)\left(1_{s} \odot \psi \odot 1_{t t}\right)\left(\zeta \odot 1_{s t t}\right)\left(1_{s} \odot e \odot 1_{t}\right) \\
=\left(1_{s s} \odot \mu\right)\left(1_{s} \odot \psi \odot 1_{t}\right)\left(\zeta \odot 1_{s t}\right)\left(1_{s} \odot\left(1_{s s} \odot \mu\right)\left(e \odot 1_{t}\right)\right) \\
\stackrel{(8.12)}{=}\left(1_{s s} \odot \mu\right)\left(1_{s} \odot \psi \odot 1_{t}\right)\left(1_{s t s} \odot \mu\right)\left(\zeta \odot 1_{s t s}\right)\left(1_{s s} \odot \psi \odot 1_{t}\right) \\
\quad\left(1_{s} \odot \psi \odot 1_{s t}\right)\left(1_{s t} \odot e\right) \\
=\left(1_{s s} \odot \mu\left(1_{t} \odot \mu\right)\right)\left(1_{s} \odot\left(\psi \odot 1_{t}\right)\left(1_{t} \odot \psi\right) \odot 1_{t}\right)\left(\zeta \odot 1_{t s t}\right) \\
\quad\left(1_{s} \odot \psi \odot 1_{s t}\right)\left(1_{s t} \odot e\right) \\
\stackrel{(8.1)}{=}\left(1_{s} \odot\left(1_{s} \odot \mu\right)\left(\psi \odot 1_{t}\right)\left(\mu \odot 1_{s t}\right)\right)\left(\left(\zeta \odot 1_{t}\right)\left(1_{s} \odot \psi\right) \odot 1_{s t}\right)\left(1_{s t} \odot e\right),
\end{gathered}
$$

as required. So our proof is complete.

We end this section by specializing Theorems 8.6 and 8.7 to the case when $\mathcal{K}=\mathcal{C}$, a monoidal category regarded as an one object 2-category. It this situation a wreath in $\mathcal{C}$ is a pair $(A, X)$ with $A$ an algebra in $\mathcal{C}$ and $(X, \psi, \zeta, \sigma)$ an algebra $\mathcal{T}_{A}^{\# \text {, }}$ see [8]. Here $\mathcal{T}_{A}^{\#}$ denotes the monoidal category $\operatorname{EM}(\mathcal{C})(A)$, and the notation is justified by the fact that $\mathcal{T}_{A}^{\#}$ is a generalization of the category of transfer morphisms through the algebra $A$ in $\mathcal{C}, \mathcal{T}_{A}$, previously introduced by Tambara in [54]. Note that $\psi: X \otimes A \rightarrow A \otimes X, \zeta: X \otimes X \rightarrow A \otimes X$ and $\sigma: \underline{1} \rightarrow A \otimes X$ are morphisms in $\mathcal{C}$ satisfying seven compatibility relations, namely the ones in (8.1)-(8.6) specialized for this particular situation.

For a wreath $(A, X)$ in $\mathcal{C}$ we denote by $A_{\psi, \zeta, \sigma} X$ the associated wreath product. $A \#_{\psi, \zeta, \sigma} X$ is an algebra in $\mathcal{C}$ and $\sigma$ induces an algebra morphism $\iota=\underline{m}_{A}\left(\operatorname{Id}_{A} \otimes \sigma\right)$ : $A \rightarrow A \#_{\psi, \zeta, \sigma} X$ in $\mathcal{C}$. Furthermore, the wreath algebra $A \#_{\psi, \zeta, \sigma} X$ is also an $A$-ring in $\mathcal{C}$, that is an algebra in the monoidal category ${ }_{A} \mathcal{C}_{A}$, provided that $A, X$ are left coflat objects (recall from Section 2.1 that for $A$ left coflat we have denoted by ${ }_{A} \mathcal{C}_{A}$ the category of $A$-bimodules that are left coflat and left robust). In fact, we have an $A$-ring structure on $A \otimes X$ with the left $A$-module structure given by $\underline{m}_{A}$ if and only if $(A, X)$ is a wreath in $\mathcal{C}$, cf. [8]. When $A \#_{\psi, \zeta, \sigma} X$ is considered as an $A$-ring we will denote it by $A \otimes X$. Remark that this double structure is possible in view of the comments made at the beginning of the proof of Proposition 4.8.

We have now the following characterizations for Frobenius/separable wreaths in $\mathcal{C}$.

Corollary 8.8. Let $\mathcal{C}$ be a monoidal category and $(A, X, \psi, \zeta, \sigma)$ a wreath in $\mathcal{C}$. Then the following assertions are equivalent:

(i) $(A, X, \psi, \zeta, \sigma)$ is a Frobenius wreath in $\mathcal{C}$;

(ii) $(X, \psi) \in \mathcal{T}_{A}^{\#}$ is a Frobenius monoidal algebra; 
(iii) $(X, \psi)$ admits a coalgebra structure $(X, \psi, \delta, f)$ in $\mathcal{T}_{A}^{\#}$ such that $\delta$ is $X$-bilinear, that is there is a cowreath structure in $\mathcal{C}$ of the form $(A, X, \psi, \delta, f)$ such that

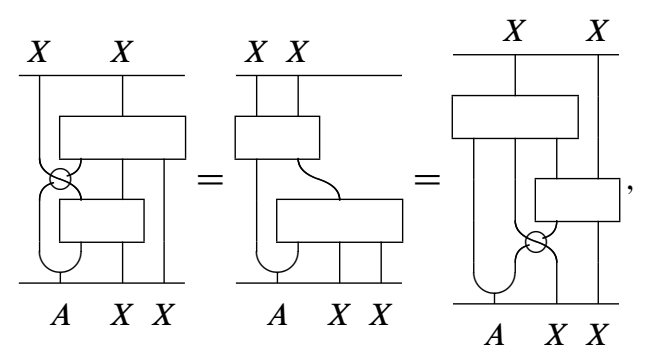

where

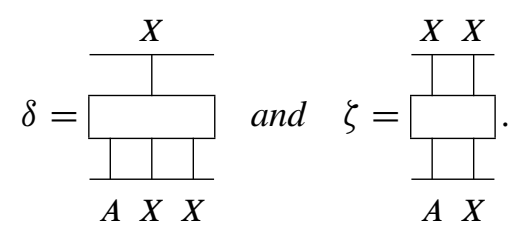

(iv) There is an adjunction $(\rho, \lambda):(X, \psi) \dashv(X, \psi)$ in $\mathcal{T}_{A}^{\#}$ with $\lambda$ associative or, otherwise stated, there exist morphisms $\rho: \underline{1} \rightarrow A \otimes X \otimes X$ and $\lambda: X \otimes X \rightarrow A$ in $\mathcal{C}$ such that the following relations are satisfied:
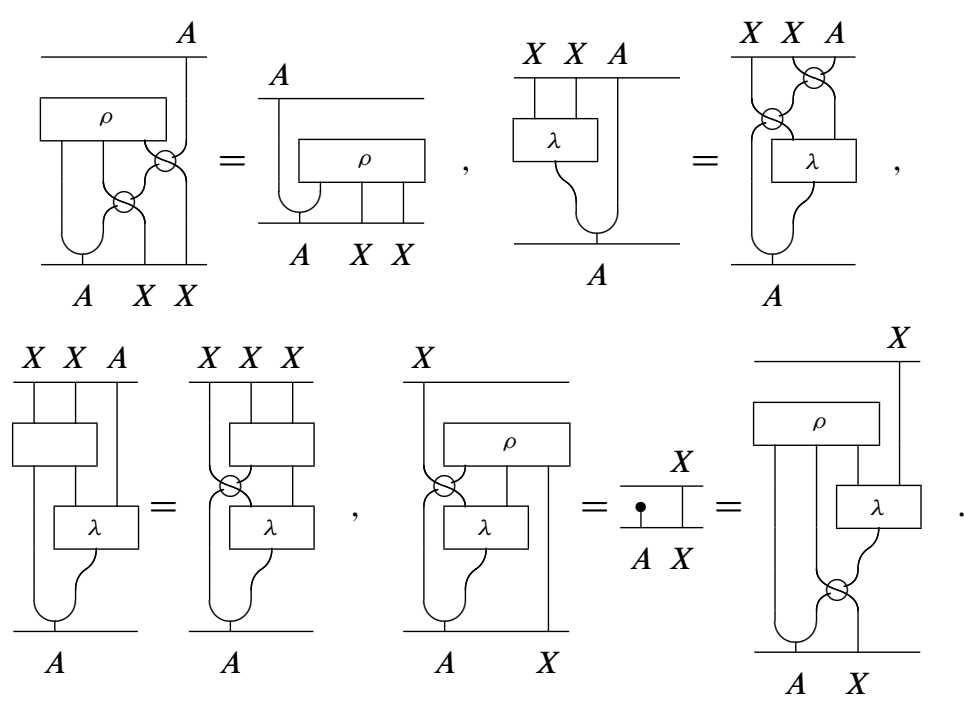

(v) There is an adjunction $(\rho, \lambda):(X, \psi) \dashv(X, \psi)$ in $\mathcal{T}_{A}^{\#}$ with $\lambda$ of the form $\lambda=\underline{m}_{A}\left(\operatorname{Id}_{A} \otimes \varsigma\right) \zeta$, for some morphism $\varsigma: X \rightarrow A$ in $\mathcal{C}$, or, in other words, there exist morphisms $\rho: \underline{1} \rightarrow A \otimes X \otimes X$ and $\varsigma: X \rightarrow A$ in $\mathcal{C}$ such that the conditions in (iv) above are fulfilled if we keep $\rho$ and replace $\lambda$ with $\underline{m}_{A}\left(\operatorname{Id}_{A} \otimes \zeta\right) \zeta$. 
If $\mathcal{C}$ admits coequalizers and any object of it is coflat then $(i)-(v)$ are equivalent to

(vi) $\iota: A \rightarrow A \#_{\psi, \zeta, \sigma} X$ is an algebra Frobenius extension in $\mathcal{C}$;

(vii) $A \otimes X$ is a Frobenius algebra in ${ }_{A} \mathcal{C}_{A}$, i.e., a Frobenius A-ring,

and if, moreover, 1 is a left $\otimes$-generator for $\mathcal{C}$ then all the statements from (i) to (vii) are also equivalent to

(viii) The functor restriction of scalars $F: \mathcal{C}_{A \#_{\psi, \zeta, \sigma} X} \rightarrow \mathcal{C}_{A}$ is a Frobenius functor.

Proof. The equivalences between (i)-(v) follow from Theorem 8.6, as well as their equivalences with (vi), provided that $\mathcal{C}$ admits coequalizers and any object of it is coflat (since, in the notations of Theorem 8.6, we have $\mathcal{K}(A)=\mathcal{C}$ ).

From Proposition 4.8 we get the desired equivalences with (vii), and with the extra hypothesis that 1 is a left $\otimes$-generator for $\mathcal{C}$ we can apply Theorem 3.5 to get the ones with (viii), respectively. Note that, since $A \otimes X$ is always left robust in $\mathcal{C}$, for the equivalences with (vii) we need only $A, X$ to be coflat objects.

Corollary 8.9. Under the hypothesis and notations of Corollary 8.8 the following statements are equivalent:

(i) $(A, X, \psi, \zeta, \sigma)$ is a separable wreath in $\mathcal{C}$;

(ii) The algebra $(X, \psi, \sigma)$ in $\mathcal{T}_{A}^{\#}$ is separable;

(iii) There exists a morphism e $: \underline{1} \rightarrow A \otimes X \otimes X$ such that
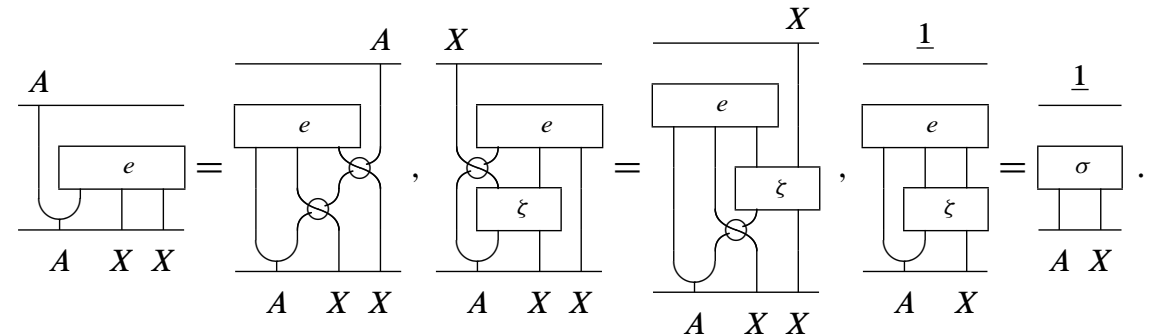

If $\mathcal{C}$ admits coequalizers and any object of it is coflat then (i)-(iii) are equivalent to

(iv) $\iota: A \rightarrow A_{\psi, \zeta, \sigma} X$ is a separable algebra extension in $\mathcal{C}$;

(iiv) $A \otimes X$ is a separable algebra in ${ }_{A} \mathcal{C}_{A}$, that is, a separable $A$-ring,

and if, moreover, 1 is a left $\otimes$-generator for $\mathcal{C}$ then $(i)-(v)$ are also equivalent to

(vi) The restriction of scalars functor $F: \mathcal{C}_{A \#_{\psi, \zeta, \sigma} X} \rightarrow \mathcal{C}_{A}$ is separable.

Proof. The equivalences between (i), (ii) and (iv) follow by specializing Theorem 8.7 for the case when $\mathcal{K}$ is $\mathcal{C}$, a monoidal category. The statement (iii) is an explicit description of the separability morphism of a separable algebra in $\mathcal{T}_{A}^{\#}$. Finally, the equivalences of (i)-(iv) with (v) and (vi) follow because of Proposition 4.8 and Theorem 3.5, respectively. Notice that, as in the Frobenius case, since $A \otimes X$ is always left robust in $\mathcal{C}$, for the equivalences with (v) we need only $A, X$ to be coflat objects. 
In [9] we will apply the results in the last two corollaries to the wreath extensions produced by generalized entwining structures, previously introduced in [8]. As a consequence we will obtain a set of Frobenius properties and Maschke-type theorems for generalized entwined module categories. Specializing them for the contexts provided by Hopf algebras and their generalizations we get at the end most of the Frobenius properties and Maschke-type theorems known for different sorts of entwined modules. We also get new ones, specially in the case when we consider contexts coming from quasi-Hopf algebra theory.

Acknowledgements. The authors are grateful to the referee for his valuable comments, which improved a first version of this paper.

\section{References}

[1] F. Borceux, Handbook of categorical algebra I: basic category theory, Encyclopedia Math. Appl., 50, Cambridge University Press, Cambridge, 1994. Zbl 0803.18001 MR 1291599

[2] G. Böhm, F. Nill and K. Szlachányi, Weak Hopf algebras I. Integral theory and $C^{*}$-structure, J. Algebra 221 (1999), 385-438. Zbl 0949.16037 MR 1726707

[3] R. Brauer and C. Nesbitt, On the regular representations of algebras, Proc. Nat. Acad. Sci. USA (1937) 23, 236-240. Zbl 0016.34102

[4] D. Bulacu, The weak braided Hopf algebra structures of some Cayley-Dickson algebras, J. Algebra 322 (2009), 2404-2427. Zbl 1247.17001 MR 2553686

[5] D. Bulacu, A Clifford algebra is a weak Hopf algebra in a suitable symmetric monoidal category, J. Algebra 332 (2011), 244-284. Zbl 1245.16023 MR 2774687

[6] D. Bulacu, Algebras and coalgebras in braided monoidal categories, Editura Universităţii Bucureşti, 2009.

[7] D. Bulacu and S. Caenepeel, Corings in monoidal categories in New techniques in Hopf algebras and graded ring theory, 53-78, K. Vlaam. Acad. België Wet. Kunsten (KVAB), Brussels, 2007. Zbl 1156.16028 MR 2395766

[8] D. Bulacu and S. Caenepeel, Monoidal ring and coring structures obtained from wreaths and cowreaths, Algebr. Represent. Theory 17 (2014), 1035-1082. Zbl 06352042 MR 3228475

[9] D. Bulacu, S. Caenepeel and B. Torrecillas, Frobenius and separable functors for the category of generalized entwined modules. Applications, preprint, 2015. 
[10] S. Caenepeel, G. Militaru and S. Zhu, Frobenius and Separable Functors for Generalized Module Categories and Nonlinear Equations, Springer Lec. Notes in Math., 1787, Springer, 2002. Zbl 1008.16036 MR 1926102

[11] S. Caenepeel and M. De Lombaerde, A categorical approach to Turaev's Hopf group-coalgebras, Comm. Algebra 34 (2006), 2631-2657. Zbl 1103.16024 MR 2240397

[12] B. Coecke, D. Pavlovic and J. Vicary, A new description of orthogonal bases, Mathematical Structures in Computer Science 23 (2013), 555-567. Zbl 1276.46016 MR 3062875

[13] B. Day and C. Pastro, Note on Frobenius monoidal functors, New York J. Math. 14 (2008), 733-742. Zbl 1178.18006 MR 2465800

[14] S. Dăscălescu, C. Năstăsescu and L. Năstăsescu, Frobenius algebras of corepresentations and group-graded vector spaces, J. Algebra 406 (2014), 226250. Zbl 06335129 MR 3188336

[15] J. Dieudonné, Remarks on quasi-Frobenius rings, Illinois Journal of Mathematics 2 (1958), 346-354. Zbl 0101.02701 MR 97427

[16] L. Dixon and A. Kissinger, Monoidal Categories, Graphical Reasoning, and Quantum Computation, CAM-CAD, 2009.

[17] S. Eilenberg and T. Nakayama, On the dimensions of modules and algebras II. (Frobenius algebras and quasi-Frobenius rings), Nagoya Math. J. 9 (1955), 1-16. Zbl 0068.26503 MR 73577

[18] S. Endo and Y. Watanabe, On separable algebras over a commutative ring, Osaka J. Math. 4 (1967), 233-242. Zbl 0189.03303 MR 227211

[19] J. Fuchs and C. Schweigert, Category theory for conformal boundary conditions. Vertex operator algebras in mathematics and physics (Toronto, ON, 2000), 25-70, Fields Inst. Commun., 39, Amer. Math. Soc., Providence, RI, 2003 Zbl 1084.17012 MR 2029790

[20] J. Fuchs and C. Stigner, On Frobenius algebras in rigid monoidal categories, Arab. J. Sci. Eng. Sect. C Theme Issues 33 (2008), no. 2, 175-191. Zbl 1185.18007 MR 2500035

[21] L. Kadison, New examples of Frobenius extensions, University Lecture Series, 14, American Mathematical Society, Providence, Rhode Island, 1999. Zbl 0929.16036 MR 1690111

[22] F. Kasch, Grundlagen einer Theorie der Frobeniuserweiterungen, Math. Annalen 127 (1954), 453-474. Zbl 0055.26301 MR 62724

[23] F. Kasch, Projective Frobenius-Erweiterungen, S.-B. Heidelberger Akad. Wiss. Math.-Nat. Kl. 1960/61 (1961), 87-109. Zbl 0104.26201 MR 132085 
[24] F. Kasch, Dualitätseigenschaften von Frobenius Erweiterungen, Math. Zeit. 77 (1961), 219-227. Zbl 0112.26502 MR 157999

[25] C. Kassel, Quantum Groups, Graduate Texts in Mathematics, 155, Springer Verlag, Berlin, 1995. Zbl 0808.17003 MR 1321145

[26] J. Kock, Frobenius algebras and $2 D$ topological quantum field theories, London Math. Soc. Stud. Text, 59, Cambridge Univ. Press, Cambridge, 2004. Zbl 1046.57001 MR 2037238

[27] S. Lack and R. Street, The formal theory of monads II, J. Pure Appl. Algebra 175 (2002), 243-265. Zbl 1019.18002 MR 1935981

[28] T. Y. Lam, Lectures on Modules and rings, Graduate Texts in Mathematics, 189, Springer-Verlag, Berlin, 1999. Zbl 0911.16001 MR 1653294

[29] A. D. Lauda, Frobenius algebras and ambidextrous adjunctions, Theory and Applications of Categories 16 (2006), 84-122. Zbl 1092.18003 MR 2210667

[30] S. Majid, Algebras and Hopf algebras in braided categories in Advances in Hopf Algebras, 55-105, Lect. Notes Pure Appl. Math.,158, Dekker, New York, 1994. Zbl 0812.18004 MR 1289422

[31] S. MacLane, Categories for the Working Mathematician, Graduate Texts in Mathematics, 5, Springer-Verlag, 1974. Zbl 0232.18001 MR 1712872

[32] K. Morita, Adjoint pairs of functors and Frobenius extensions, Sc. Rep. T.K.D. Sect. A 9 (1965), 40-71. Zbl 0163.28602 MR 190183

[33] K. Morita, The endomorphism ring theorem for Frobenius extensions, Math. Zeit. 102 (1967), 385-404. Zbl 0162.33803 MR 224658

[34] M. Müger, From sufactors to categories and topology I. Frobenius algebras in and Morita equivalence of tensor categories, J. Pure Appl. Alg. 180 (2003), 81-157. Zbl 1033.18002 MR 1966524

[35] T. Nakayama, On Frobeniusean algebras. I, Annals of Mathematics. Second Series (Annals of Mathematics) 40 (1939), 611-633. Zbl 0021.29402 MR 16

[36] T. Nakayama, Note on symmetric algebras, Ann. of Math. 39 (1938), 659-668. Zbl 0019.10202 MR 1503430

[37] T. Nakayama, On Frobeniusean algebras. II, Annals of Mathematics. Second Series (Annals of Mathematics) 42 (1941), 1-21. Zbl 67.0092.04 MR 4237

[38] T. Nakayama and T. Tsuzuku, A Remark on Frobenius extensions and endomorphism rings I, Nagoya Math. J. 17 (1960), 89-110. Zbl 0204.05101 MR 124375

[39] T. Nakayama and T. Tsuzuku, A Remark on Frobenius extensions and endomorphism rings II, Nagoya Math. J. 19 (1961), 127-148. Zbl 0204.05102 MR 140540 
[40] T. Nakayama and T. Tsuzuku, Correction to our paper "On Frobenius extensions. II", Nagoya Math. J. 20 (1962), 205-205. Zbl 0204.05102 MR 140541

[41] C. Năstăsescu, M. Van den Bergh and F. Van Oystayen, Separable functors applied to graded rings, J. Algebra 123 (1989), 397-413. Zbl 0673.16026 MR 1000494

[42] B. Pareigis, Einige Bemerkung über Frobeniuserweiterungen, Math. Ann. 153 (1964), 1-13. MR 166222

[43] B. Pareigis, Vergessende Funktoren and Ringhomomorphismen, Math. Zeit. 93 (1966), 265-275. Zbl 0146.02503 MR 202804

[44] B. Pareigis, Non-additive ring and module theory V. Projective and coflat objects, Algebra Ber. 40 (1980). Zbl 0484.18007

[45] D. M. Rafael, Separable functors revisited, Comm. Alg. 18 (1990), 1445-1459. Zbl 0713.18002 MR 1059740

[46] P. Schauenburg, Actions on monoidal categories and generalized Hopf smash products, J. Algebra 270 (2003), 521-563. Zbl 1041.16030 MR 2019629

[47] P. Schauenburg, Weak Hopf algebras and quantum groupoids, Noncommutative geometry and quantum groups (Warsaw, 2001), 171-188, Banach Center Publ., 61, Polish Acad. Sci., Warsaw, 2003. Zbl 1064.16041 MR 2024429

[48] R. Street, Frobenius Monads and Pseudomonoids, J. Math. Phys. 45 (2004), 3930-3948. Zbl 1071.18006 MR 2095680

[49] R. Street, Frobenius algebras and monoidal categories, Annual Meeting Aust. Math. Soc. September 2004.

[50] K. Sugano, Separable extensions and Frobenius extensions, Osaka J. Math. 7 (1970), 291-299. Zbl 0213.04402 MR 289577

[51] K. Sugano, Note on separability of endomorphism rings, J. Fac. Sci. Hokkaido Univ. 21 (1971), 196-208. Zbl 0236.16003 MR 294395

[52] K. Szlachányi, Finite quantum groupoids and inclusions of finite type, Mathematical physics in mathematics and physics (Siena, 2000), 393407, Fields Inst. Commun., 30, Amer. Math. Soc., Providence, RI, 2001. Zbl 1022.18007 MR 1867570

[53] K. Szlachányi, Adjointable monoidal functors and quantum groupoids, Hopf algebras in noncommutative geometry and physics, 291-307, Lecture Notes in Pure and Appl. Math., 239, Dekker, New York, 2005. Zbl 1064.18008 MR 2106937

[54] D. Tambara, The coendomorphism bialgebra of an algebra, J. Fac. Sci. Univ. Tokyo Sect. IA Math. 37 (1990), no. 2, 425-456. Zbl 0717.16030 MR 1071429 
[55] S. Yamagami, Frobenius algebras in tensor categories and bimodule extensions, Galois theory, Hopf algebras, and semiabelian categories, 551-570, Fields Inst. Commun., 43, Amer. Math. Soc., Providence, RI, 2004 Zbl 1079.18004 MR 2075605

[56] R. Wisbauer, Modules and algebras: bimodule structure and group actions on algebras, Pitman Mon. Pure Appl. Math., 81, Addison Wesley Longman, Harlow, Essex, 1996. Zbl 0861.16001 MR 1396313

Received 24 September, 2013; revised 10 July, 2014

D. Bulacu, Faculty of Mathematics and Informatics, University of Bucharest,

Str. Academiei 14, RO-010014 Bucharest 1, Romania

E-mail: daniel.bulacu@fmi.unibuc.ro

B. Torrecillas, Department of Algebra and Analysis, Universidad de Almería, E-04071 Almería, Spain

E-mail: btorreci@ual.es 